\title{
Additional in-series compliance does not affect the length dependence of activation in rat medial gastrocnemius
}

\section{MacDougall, Keenan Barry}

MacDougall, K. B. (2020). Additional in-series compliance does not affect the length dependence of activation in rat medial gastrocnemius (Unpublished master's thesis). University of Calgary, Calgary, AB.

http://hdl.handle.net/1880/111768

Downloaded from PRISM Repository, University of Calgary 


\title{
UNIVERSITY OF CALGARY
}

Additional in-series compliance does not affect the length dependence of activation in rat medial gastrocnemius

by

Keenan Barry MacDougall

\author{
A THESIS \\ SUBMITTED TO THE FACULTY OF GRADUATE STUDIES \\ IN PARTIAL FULFILMENT OF THE REQUIREMENTS FOR THE \\ DEGREE OF MASTER OF SCIENCE
}

GRADUATE PROGRAM IN KINESIOLOGY

CALGARY, ALBERTA

MARCH, 2020

(C) Keenan Barry MacDougall 2020 


\begin{abstract}
Background: The force-length relationship describes the amount of force a muscle can produce as a function of its length, typically during maximal isometric contractions. When activation is submaximal, it has been shown that the length at which force production is highest (the optimum length) is shifted towards longer lengths. The usual explanation for this shift is a length-dependent increase in calcium sensitivity due to an increased proximity of the myofilaments with increases in length, which has been dubbed the "length dependence of activation" (LDA). Some recent research has suggested that shortening-induced force depression, (a phenomenon which describes the relative reduction in muscle force when a muscle is actively shortening to a given length compared to contracting isometrically at that same length) has the potential to alter a muscle's optimum length in an activation-dependent way, thereby affecting any activation dependent shifts in optimum length.
\end{abstract}

Purpose: The purpose of this study was to determine whether shortening-induced force depression would impact the relative shift in optimum length that occurs with submaximal activation.

Methods: Rat medial gastrocnemius muscle was isolated and stimulated at $200 \mathrm{~Hz}$ triplet pulses and single pulse stimulation over a range of lengths, both with and without additional in-series compliance provided by a small piece of silicon tubing in series with the muscle tendon unit. Peak force and optimum length were calculated by curve-fitting of the force-length data, and these data were compared between low- and high compliance conditions as well as with maximal and submaximal activation.

Results: Additional in-series compliance led to a significant decrease in force at both maximal and submaximal activation, but did not impact the optimum length for either condition. Therefore, in our model, shortening-induced force depression did not impact the length dependence of activation.

Conclusion: The additional in-series compliance allowed greater muscle shortening upon activation, which was accompanied by a reduction in force, however the post-shortening length at which the muscle produced the most force was unchanged. The best explanation for the length dependence of activation in skeletal muscle remains a length dependent increase in calcium sensitivity. 


\section{Preface}

This thesis is original, unpublished, work by the author, K. MacDougall. The experiments reported in Chapters 2-4 were approved by the University of Calgary Life and Environmental Sciences Animal Care Committee for the project "Skeletal Muscle Force Modulation: Length

Dependent Factors". The certificate, Ethics ID AC16-0193, was renewed on October 25, 2019 and is presented in Appendix B. 


\section{Acknowledgements}

Walter and Doug, thank you both for serving on my supervisory committee. Along with Brian, I feel like I lucked out with the "Dream Team" committee. I can't imagine that there would be many institutions in the world where one could be led by such well-respected scientists as all of you are, and for that I am very thankful.

Anders, thank you for all of your help with much of the preliminary work, and for allowing me to be a part of some of your work. It was a great experience, and I hope the future might bring our work together again!

Andrew, thank you for your help in the laboratory, and taking time out of your day to tend to my simple questions. It is much appreciated.

Finally, thank you Brian for taking me on as a student, and being so supportive along the journey. I entered into this program at the University of Calgary having only a modest idea of where my interests really were, and I feel incredibly fortunate to have been able to fall into the wonderful world of muscle research with you as an advisor. Here's hoping that this thesis is only the beginning. 


\section{Table of Contents}

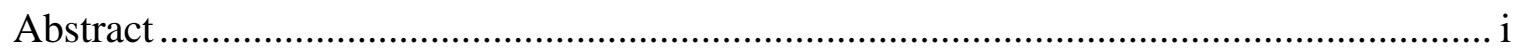

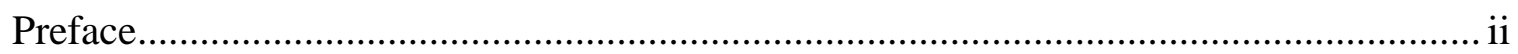

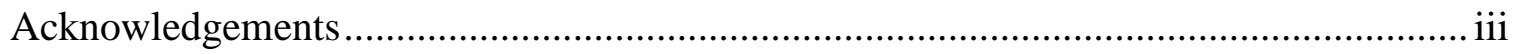

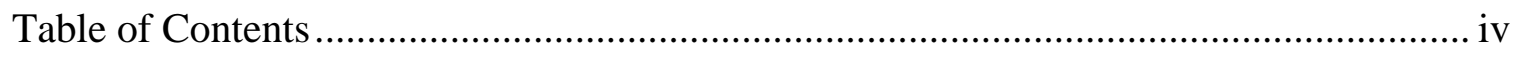

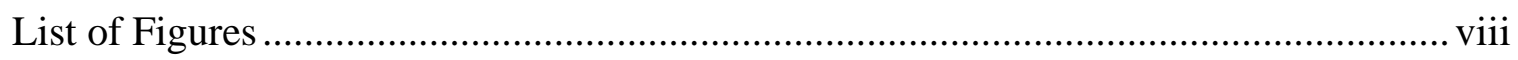

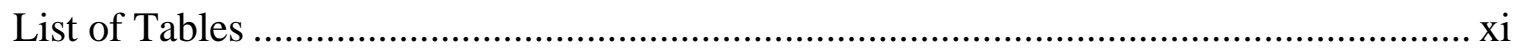

List of symbols, abbreviations, and nomenclature .................................................. xii

1 Muscle 101: The Basis of Muscle Contraction ............................................................. 1

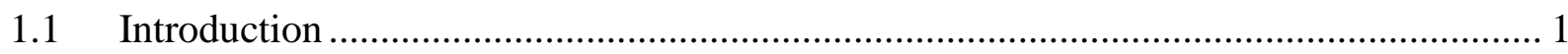

1.2 Structure of Muscle .......................................................................................... 1

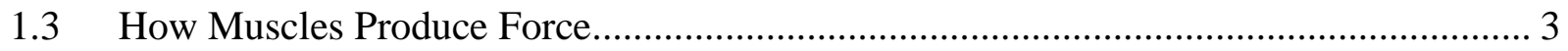

1.4 Determinants of Muscle Force Output .............................................................. 5

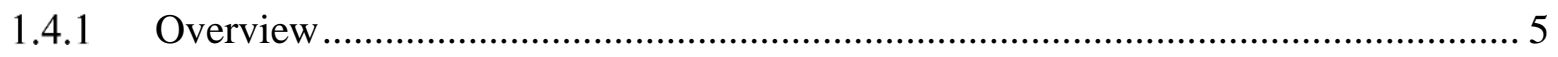

1.4.2 How Activation Level Impacts Muscle Force Production.................................. 6

1.4.3 How the Relative Length of a Muscle Impacts Force Production ......................... 8

1.4.4 How Velocity of Shortening Impacts Muscle Force Production ......................... 10

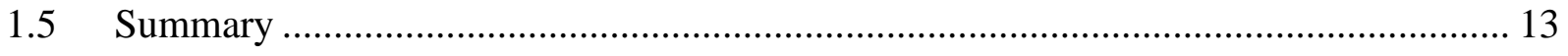

2 The Determinants of the Optimum Length of Muscle ................................................ 15 
2.1 Measuring the Optimum length: Absolute length, Relative Length, and the Potential for Variances in the Force-Length Relationship 15

2.2 A Brief History of the Length Dependence of Activation ........................................ 19

2.3 Mechanisms of LDA in skeletal muscle ............................................................. 20

2.3.1 The Effects of $\mathrm{Ca}^{2+}$ Sensitivity on Activation-Dependent Shifts in Optimum

Length: The Case for Proximity of Myofilaments................................................... 20

2.3.2 $\mathrm{Ca}^{2+}$ Independent Effects on the Activation-Dependent Shift in Optimum Length 27

2.4 Shortening-Induced Force Depression and its Potential Role in Determining Skeletal

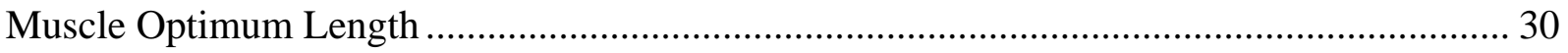

2.4.1 History and Mechanisms of Shortening-Induced Force Depression .................... 30

2.4.2 Can Shortening-Induced Force Depression Impact Optimum Length?................ 33

2.4.3 Does Increased Compliance Cause Shortening-Induced Force Depression? ......... 36

2.5 The Potential Role of the Method of Calculation of Active Force on Activation-

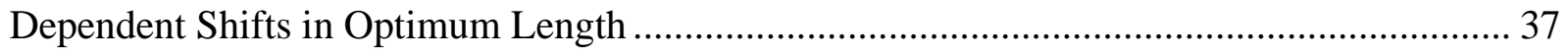

3 Addressing the Problem: Can Shortening-Induced Force Depression Explain Activation-

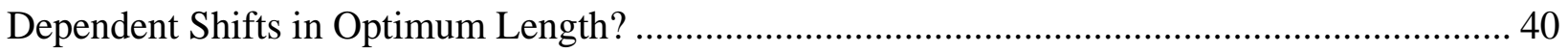

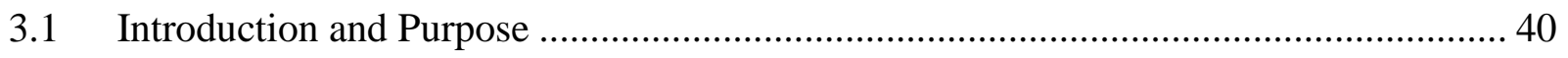

3.2 Hypotheses and Formulation of Experimental Design ....................................... 41

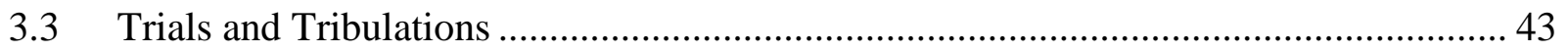

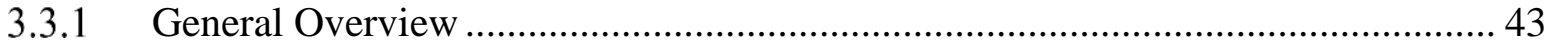

3.3.2 The "Sweet Spot" of Additional Compliance .................................................. 44 
3.3.3 Fatigue and/or Unexplained Losses in Force ....................................................... 46

3.3.4 Nerve Cuff Connection Quality ....................................................................... 48

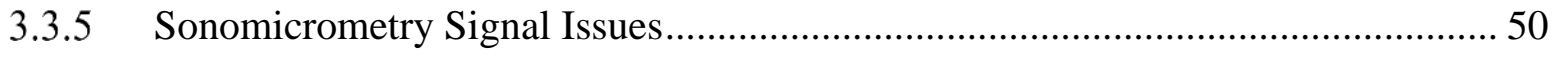

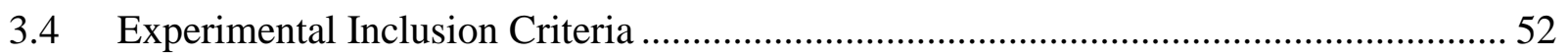

3.5 Preliminary Experimental Methods, Results and Discussion ...................................... 53

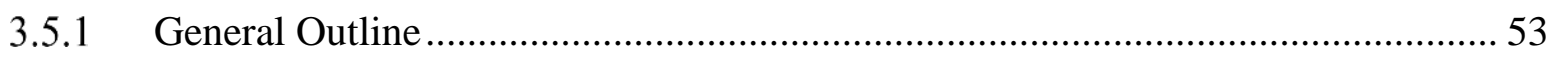

3.5.2 Experimental Protocol Overview................................................................... 53

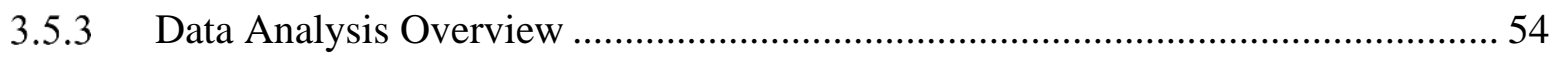

3.5.4 Stimulation Protocol Version 1....................................................................... 54

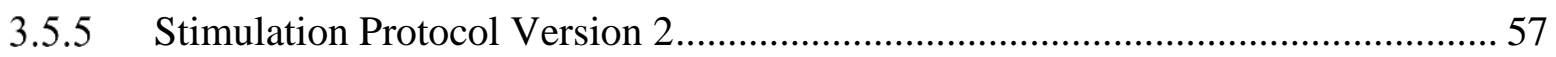

3.5.6 Combined Data from Stimulation Protocol Versions 1 and 2 .............................. 59

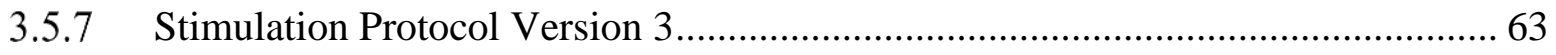

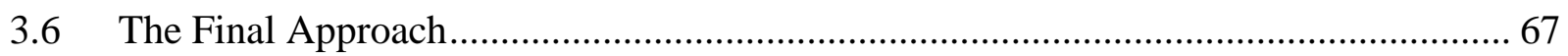

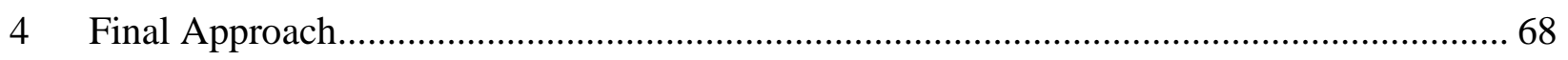

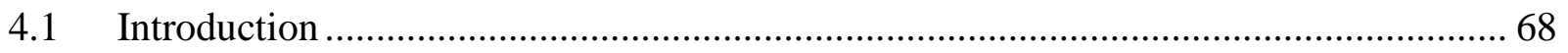

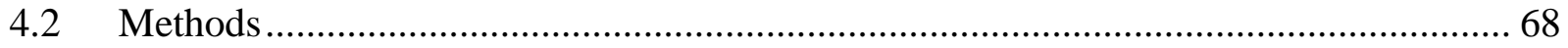

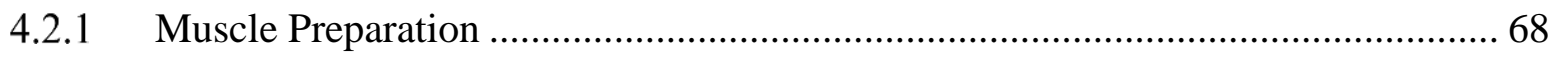

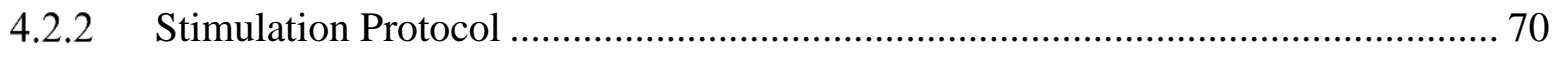

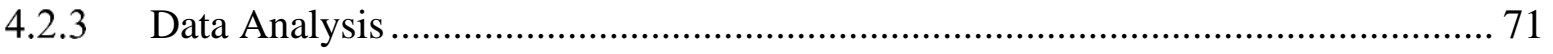

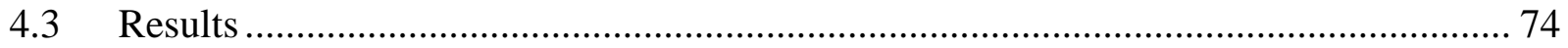




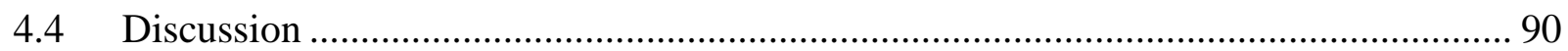

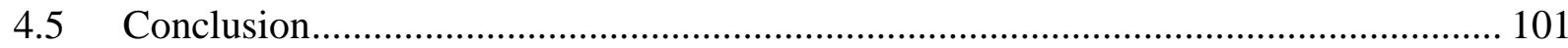

5 Conclusions, Limitations, and Future Directions .................................................... 95

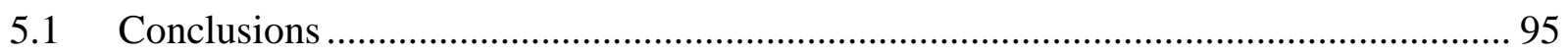

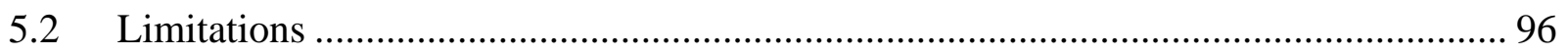

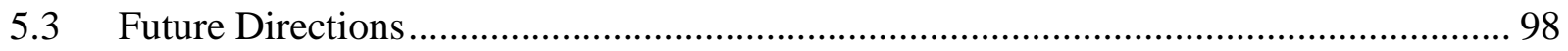

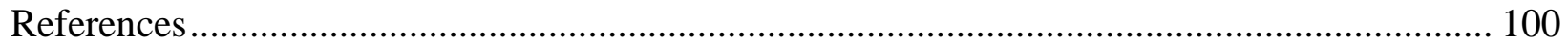

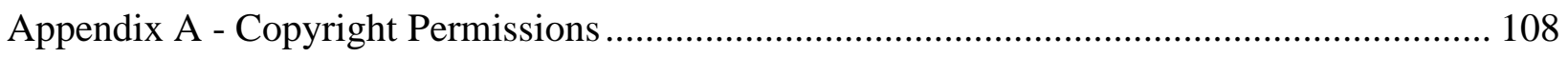




\section{List of Figures}

Figure 1.1 The structure of muscle. ................................................................................. 2

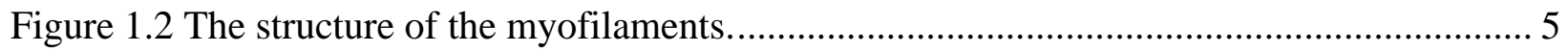

Figure 1.3 Force-frequency relationship of rat medial gastrocnemius. ................................... 7

Figure 1.4 Schematic illustration relating the degree of filament overlap and corresponding

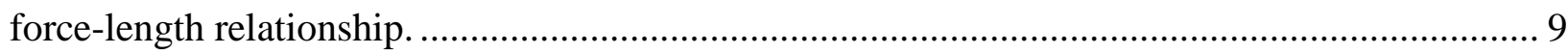

Figure $1.5 \quad$ Force-velocity relationship of frog sartorius............................................. 11

Figure 1.6 Intracellular $\mathrm{Ca}^{2+}$ transient and force during a twitch in frog muscle.................. 13

Figure 2.1 The influence of temperature on the force-length relationship............................ 21

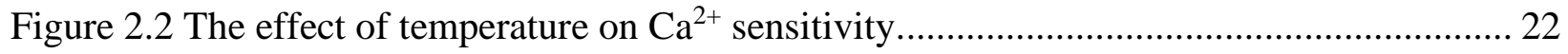

Figure 2.3 The effect of sarcomere length on $\mathrm{Ca}^{2+}$ sensitivity........................................ 24

Figure 2.4 Schematic illustration of changes in filament proximity based on changes in sarcomere length, and the corresponding force-length relationship. ................................... 27

Figure 2.5 The length-dependence of force depression. .................................................. 34

Figure 2.6 The effect of shortening-induced force depression on optimum MTU length. ...... 36

Figure 2.7 The effect of the method of active force calculation on the apparent optimum

length. $\quad 39$

Figure 3.1 Theoretical illustration demonstrating how a length dependent increase in force depression may serve to increase the relative shift in optimum length. ................................ 41

Figure 3.2 The effect of extreme series compliance on the magnitude of muscle shortening

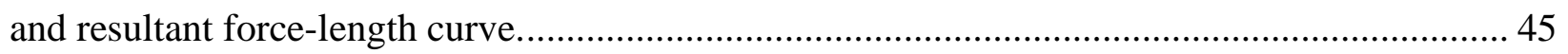

Figure 3.3 Example force-length relationship of the reduced voltage $100 \mathrm{~Hz}$ contractions.... 50

Figure 3.4 Raw data tracings of force and fascicle length. ....................................... 51 
Figure 3.5 Sample force tracings of twitch, $50 \mathrm{~Hz}, 100 \mathrm{~Hz}$ with reduced voltage, $100 \mathrm{~Hz}$, and potentiated twitch contractions. 55

Figure 3.6 Preliminary experimental data showing relative peak force and relative optimum length for twitch, $50 \mathrm{~Hz}, 100 \mathrm{~Hz}$, and potentiated twitch in low- and high compliance conditions.

60

Figure 3.7 Incomplete force-length relationship due to excessive muscle shortening........... 66

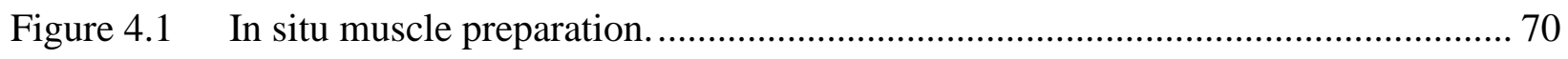

Figure 4.2 The effect of additional in-series compliance on the force-length relationships of triplet and twitch contractions

Figure 4.3 Force-length relationship of twitch and triplet contractions calculated the traditional way. 79

Figure 4.4 Work performed as a function of muscle length............................................ 80

Figure 4.5 Raw data tracings showing the effect of increased compliance on the force output and magnitude of shortening.

Figure 4.6 Sample force vs fascicle length tracings for triplet and twitch contractions at varying initial fascicle lengths. 83

Figure 4.7 Relationship between the difference in force output as a function of the difference in work performed across levels of compliance. 84 Figure 4.8 The effect of additional in-series compliance on the magnitude of shortening at varying muscle lengths. 85 Figure 4.9 Relationship between the difference in force output as a function of the difference in shortening magnitude across levels of compliance. 86 
Figure 4.10 The effect of additional in-series compliance on the velocity of shortening at

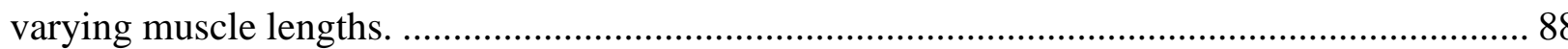

Figure 4.11 The relationship between shortening velocity and compliance at varying muscle lengths. $\quad 89$

Figure 4.12 Relationship between the difference in force output as a function of the difference in shortening velocity across levels of compliance.

Figure 4.13 The effect of increased passive force and increased compliance on the potential error in calculation of active force. 


\section{List of Tables}

Table $3.1 \quad$ Relative peak force and relative optimum length of twitch, 50Hz, submaximal voltage $100 \mathrm{~Hz}, 100 \mathrm{~Hz}$, and potentiated twitch contractions with and without additional in-series compliance 56

Table 3.2 Relative peak force and relative optimum length of twitch, $50 \mathrm{~Hz}, 100 \mathrm{~Hz}$, and potentiated twitch contractions with and without additional in-series compliance 58

Table 3.3 Relative peak force and relative optimum length of twitch, $50 \mathrm{~Hz}, 100 \mathrm{~Hz}$, and potentiated twitch contractions with and without additional in-series compliance 59

Table 3.4 Relative peak force and optimum length for twitch and $100 \mathrm{~Hz}$ contractions....... 64

Table 4.1 Relative optimum length, peak force, and compliance for different activation and compliance conditions

Table 4.2 Mean estimated optimum length for different activation and compliance conditions calculated both with and without taking fascicle shortening into account. 77

Table 4.3 Mean work done at optimum length, mean relative optimum length, and mean relative peak force between $H F-L C, H F-H C$ and $R F-L C$ conditions. 81

Table 4.4 Mean shortening velocity at optimum length for different activation and compliance conditions 87 Table 4.5 Relative optimum length values from Figure 4.10, comparing the effect of the method of calculation of active force on the optimum length while manipulating passive force and compliance 


\section{List of symbols, abbreviations, and nomenclature}

ATP - Adenosine Triphosphate

LDA - Length dependence of activation

MTU - Muscle Tendon Unit

HF-LC - High force, low compliance (in our experiments, $200 \mathrm{~Hz}$ triplet contractions without additional in-series compliance)

HF-HC - High force, high compliance (in our experiments, $200 \mathrm{~Hz}$ triplet contractions with additional in-series compliance provided by a small piece of silicon tubing)

LF-LC - Low force, low compliance (in our experiments, twitch contractions without additional in-series compliance)

LF-HC - Low force, high compliance (in our experiments, twitch contractions with additional in-series compliance provided by a small piece of silicon tubing)

Lo - Optimum length

RLC - Myosin regulatory light chain

Traditional method of active force calculation - Subtracting the passive force present prior to the onset of activation from the total force exerted by the muscle

Alternative method of active force calculation - Subtracting the passive force estimated to be present at the final post-shortening length from the total force exerted by the muscle 


\section{Muscle 101: The Basis of Muscle Contraction}

\subsection{Introduction}

Skeletal muscle tissue is an incredibly fascinating material to study, not only due to its supreme importance in our daily function, but also because there is still so much that we don't know about it. In the last couple of centuries, muscle is likely amongst the most well-studied biological material, but we are still constantly finding out new things about how it functions. This chapter will form a (very) brief introduction as to how muscles work on a fundamental level before exploring the effects of additional in-series compliance on the activation-dependent shift in optimum length.

\subsection{Structure of Muscle}

When we think of the structure of muscle, we might imagine a cut of meat from a butcher - tough, fibrous tissue with a pink or reddish color. As we look closer, we can see a hierarchical organization to muscle tissue, whereby each successive "layer" is assembled almost like a Russian doll, until we get all the way to the proteins that create the building blocks for muscle. Whole muscle is composed of bundles of muscle tissue called fascicles that are all sheathed in their own connective tissue, the perimysium (Boulpaep et al., 2009). Each of these fascicles are made up of numerous muscle fibres, which are the individual cells of the muscle. Looking deeper, each muscle fibre is filled with many cylindrical structures called myofibrils, which contain the proteins that allow muscles to produce force. Myofibrils contain proteins such as actin, myosin, and titin (among many other regulatory proteins) that are organized in a regular array which allows muscle to change its length and produce force, allowing us to move (Boulpaep et al., 2009). Actin and myosin filaments overlap with one another, and essentially "slide" past one another when the muscle changes in length (Huxley \& Niedergerke, 1954; 
Huxley \& Hanson, 1954). This notion has been termed the "sliding filament theory." These filamentous proteins are organized into repeating segments called sarcomeres, which are often referred to as the "functional unit" of the muscle cell. The myofibrils within the muscle cells are composed of many sarcomeres in series with one another, all of which have the capacity to produce force and change their length upon activation (Boulpaep et al., 2009). See Figure 1.1 below for an illustration of the structure of muscle.

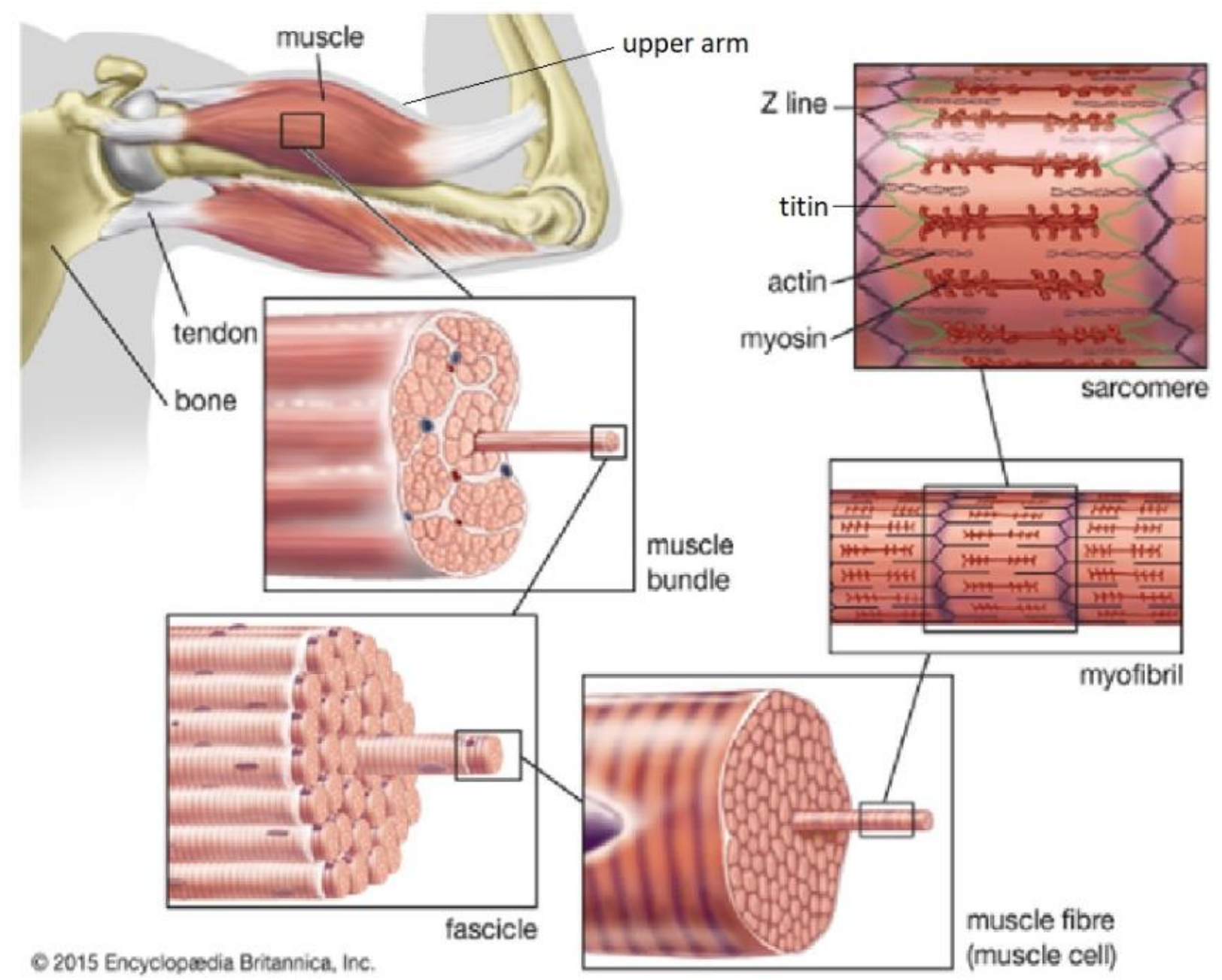

Figure 1.1 The structure of muscle.

Illustration of the hierarchical structure of muscle, from a macro level, showing the outward appearance of muscle and tendon structure, to the micro level, showing individual muscle fibres and sarcomeres. By courtesy of Encyclopædia Britannica, Inc., copyright 2015; used with permission. 


\subsection{How Muscles Produce Force}

As described above, muscle is composed of numerous types of proteins that all fit together and function cohesively to allow muscle to generate force and change length. The key proteins involved in this complex orchestra are actin, myosin, troponin, tropomyosin, and titin. Actin and myosin can exist both as "individual" molecules, called monomers or as an assembly of multiple monomers attached to one another, called polymers. Within skeletal muscle, myosin is arranged as an intertwined filament of numerous myosin molecules, all of which are assembled essentially as a series of coils with two "heads" that protrude from the "backbone" of the molecule. These multiple myosin molecules make up what is referred to as the "thick filament." Similarly, actin, tropomyosin, and troponin are arranged into a long helical filament referred to as the "thin filament." Within the thin filament, the strand-like tropomyosin lies near the grooves in the actin helix (see Figure 1.2 below), and along with troponin functions to block the sites on the actin molecule to which the myosin heads can bind to form what are called crossbridges.

Before a muscle can contract and produce force, it needs to be electrically stimulated. Muscle is classified as an excitable tissue, meaning that it can respond to and propagate electrical signals. The "source" of these electrical signals is the many charged molecules included in the tissue, such as $\mathrm{Na}^{+}, \mathrm{K}^{+}, \mathrm{Cl}^{-}$, and $\mathrm{Ca}^{2+}$. The buildup and/or movement of these charged molecules can incite a cascade of events, culminating in the propagation of an electrical signal known as an action potential. When a motor neuron or muscle is stimulated, either from the nervous system or from an external source of electrical current, these action potentials will travel down to the individual muscle cells, resulting in the release of calcium from the sarcoplasmic reticulum $(S R)$, 
a structure within the muscle cells that functions to store calcium. When this calcium is released from the sarcoplasmic reticulum, it will diffuse outwards and begin to interact with other molecules within the muscle cell. One of the most important molecules that calcium binds to within the muscle cell is troponin, which facilitates the movement of tropomyosin off the myosin binding sites, allowing myosin heads to form cross-bridges between actin and myosin. When calcium is present, these myosin cross-bridges go through a process which effectively "pulls" the thin filament over the thick filament, utilizing ATP energy and producing a shortening force in the process (Boulpaep et al., 2009). This has been termed the "cross-bridge theory of muscular contraction" (AF Huxley, 1957). Titin is a very large protein present in the sarcomere which functions to keep the myosin filaments in the middle of the sarcomere, however we are finding that titin may play many other roles in muscle function as well (Herzog, 2018). 


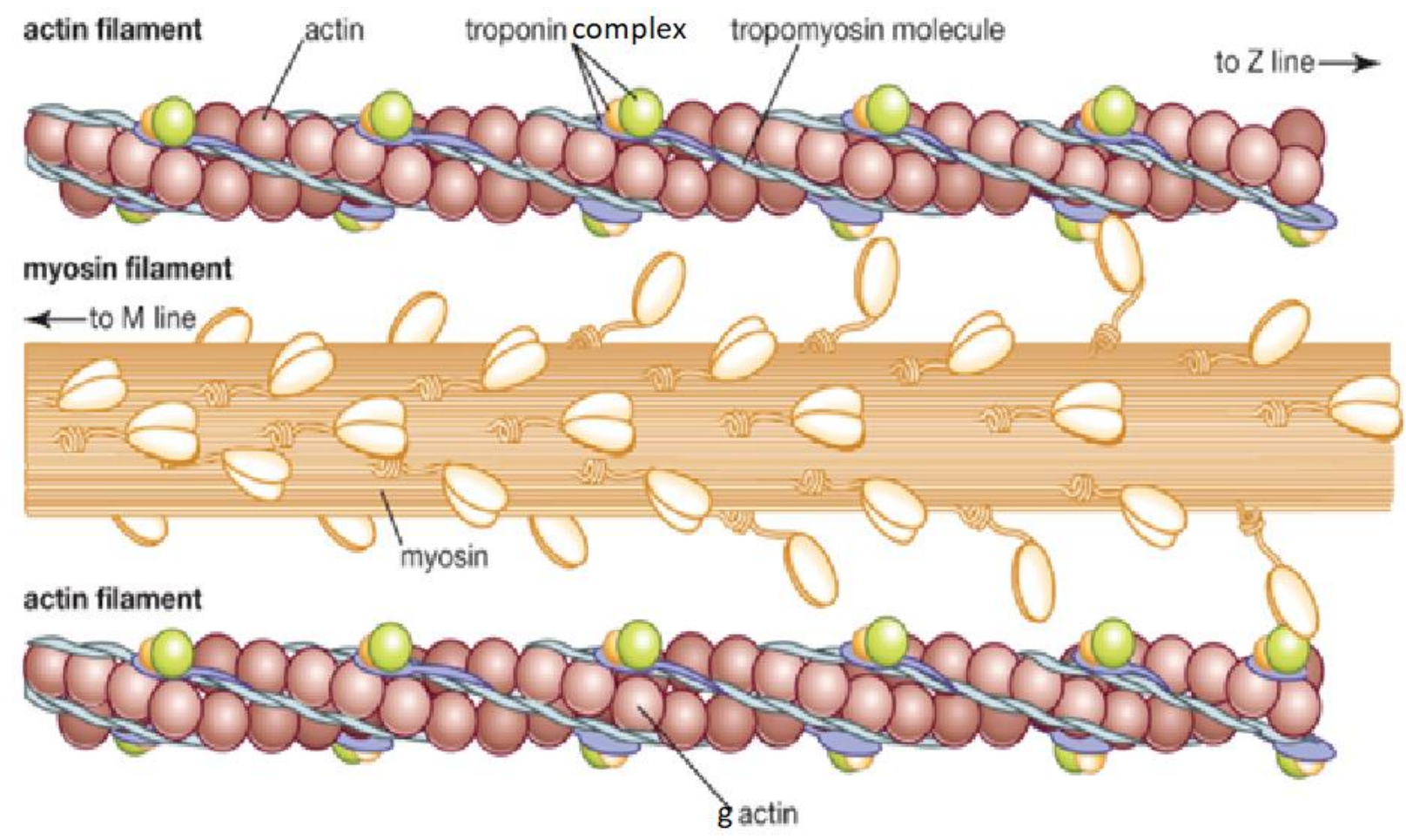

myosin molecule

$\operatorname{rod}$

heads

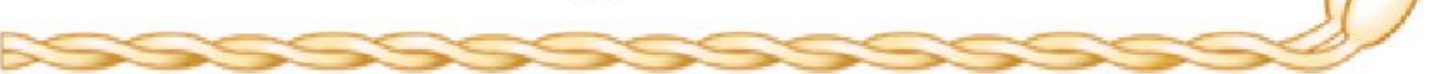

2012 Encyclopædia Britannica, Inc.

\section{Figure 1.2 The structure of the myofilaments.}

Illustration of the different proteins that make up the thick and thin filaments. The thin filament is composed of actin molecules arranged in a double helical pattern along with troponin, and tropomyosin, while the thick filament is composed of myosin molecules arranged in a double helical pattern with the myosin "heads" protruding out from the protein's "backbone." Note the variation in myosin head proximity to the thin filament, based on how far it is extending out from the backbone. By courtesy of Encyclopædia Britannica, Inc., copyright 2012; used with permission.

\subsection{Determinants of Muscle Force Output}

\subsubsection{Overview}

As described above, when calcium is released in the muscle, the myosin heads are able to attach to the actin molecules forming cross-bridges, which produce the force that muscles need to move us. As cross-bridges are the force-producing structures within muscle tissue, it then 
follows that the main determinant of muscle force production is the number of cross-bridges that are able to form at any given instant. When muscles produce force, cross-bridges are cyclically attaching and detaching; the relative rate of attachment and detachment will dictate the number of cross-bridges at any instant in time. Put simply, more cross-bridges simultaneously bound means more force produced. From here, we need to consider the different factors that can influence the number of cross-bridges able to form. The main determinants of muscle force to consider: are the activation level, the muscle's speed of contraction, and finally the sarcomere length of the muscle.

\subsubsection{How Activation Level Impacts Muscle Force Production}

The body has essentially two ways to increase the force production of a given muscle.

First off, it can increase the number of muscle fibres participating in the task. For tasks requiring only small amounts of force, not all of the fibres within a muscle will be active, but this number will increase as the force requirements rise. This increased number of active muscle fibres is achieved by "motor unit recruitment," controlled by the nervous system. The second way force can be increased is by a stronger stimulus to an individual muscle cell. This is achieved by increasing the frequency of stimulation from the nervous system, which will increase the amount of calcium released within the muscle cell. While "activation" has been given numerous definitions in the context of muscle physiology, here, activation level will refer to the concentration of calcium within the muscle cell. By binding to a greater number of troponin molecules and facilitating the movement of tropomyosin off of the myosin binding sites on actin, a higher concentration of calcium within the muscle cell will allow a greater number of myosin binding sites on actin to "open up." This will increase the probability of cross-bridge formation and increase the potential for force production. Higher calcium concentrations within the muscle 
are a result of the muscle being stimulated at a faster rate by the nervous system, in a process known as rate coding (Enoka \& Duchateau, 2017). This rate, or frequency of stimulation will determine the amount of calcium released in the muscle and therefore the subsequent force production, a relationship referred to as the "force-frequency relationship" (see Figure 1.3 below). Since it is increased calcium that is allowing greater force production to occur, and the calcium release is dependent upon the frequency of stimulation, this force-frequency relationship mirrors that of the "force-pCa" relationship; as $\left[\mathrm{Ca}^{2+}\right]$ increases, force production rises in a sigmoidal relationship until it plateaus, a level at which the calcium binding sites are saturated. An example of this relationship is seen in Figure 2.2 The effect of temperature on $\mathrm{Ca}^{2+}$ sensitivity.

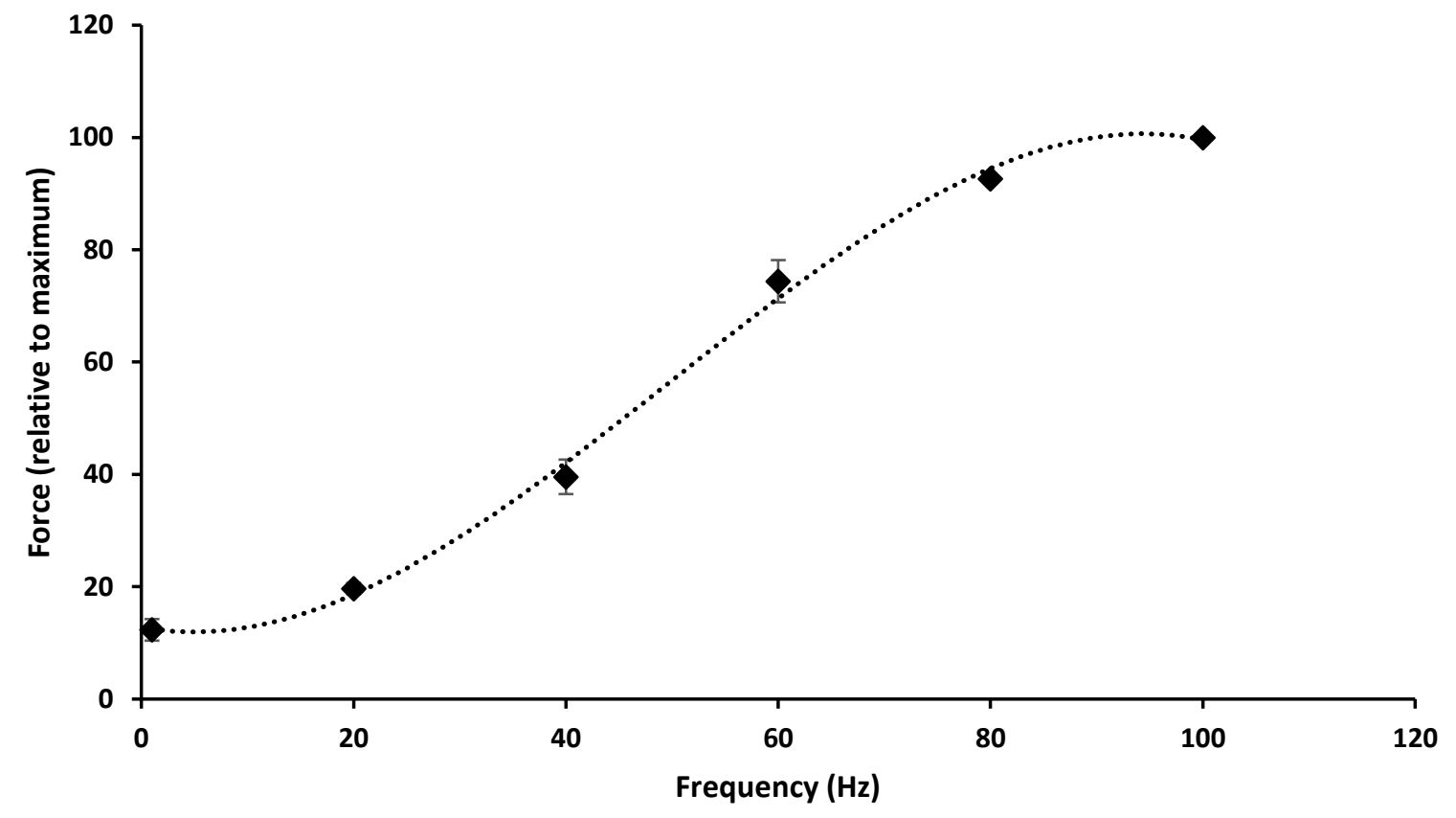

Figure 1.3 Force-frequency relationship of rat medial gastrocnemius.

Data points are mean \pm SE from 7 experiments. As stimulation frequency is increased, more calcium will be released, allowing more cross-bridges to attach and therefore more force to be produced. 


\subsubsection{How the Relative Length of a Muscle Impacts Force Production}

The relative length of a muscle is another key determinant of the amount of force it can produce. As described above, muscles are made up of overlapping protein filaments, the thick and thin filaments. The myosin molecules have globular heads, distributed along the thick filaments which can interact with the thin filaments, forming myosin cross-bridges, and thereby producing force. Since cross-bridges can only form where there is overlap between the thick and thin filaments, it follows that the number of potential cross-bridges is dependent upon the relative degree of filament overlap. Since filament overlap will depend on a muscle's relative length, then logically, the amount of force a muscle can produce is dependent on its length as well. This is referred to as the force-length relationship, which demonstrates that a muscle will be capable of producing the most force at some intermediate length, and lengths shorter and longer than this will result in less force production (see Figure 1.4). This force-length relationship has an ascending limb, a plateau, and a descending limb. While it is quite easy to visualize why the potential for force would decrease on the descending limb where filament overlap is linearly decreasing, explaining the ascending limb isn't quite as clear. While difficult to directly test, the suggested mechanisms for why force is decreased despite ample filament overlap include the thick filaments impinging on the Z-disks and the "buckling" of the myofilaments (Taylor \& Rudel, 1970) increasing the resistance to shortening, or a "double overlap" of the thin filaments, which would potentially disrupt the myosin binding sites and/or prevent cross-bridge force from effectively being exerted in the proper direction (Gordon, Huxley, \& Julian, 1966; Julian, Moss, \& Sollins, 1978). 

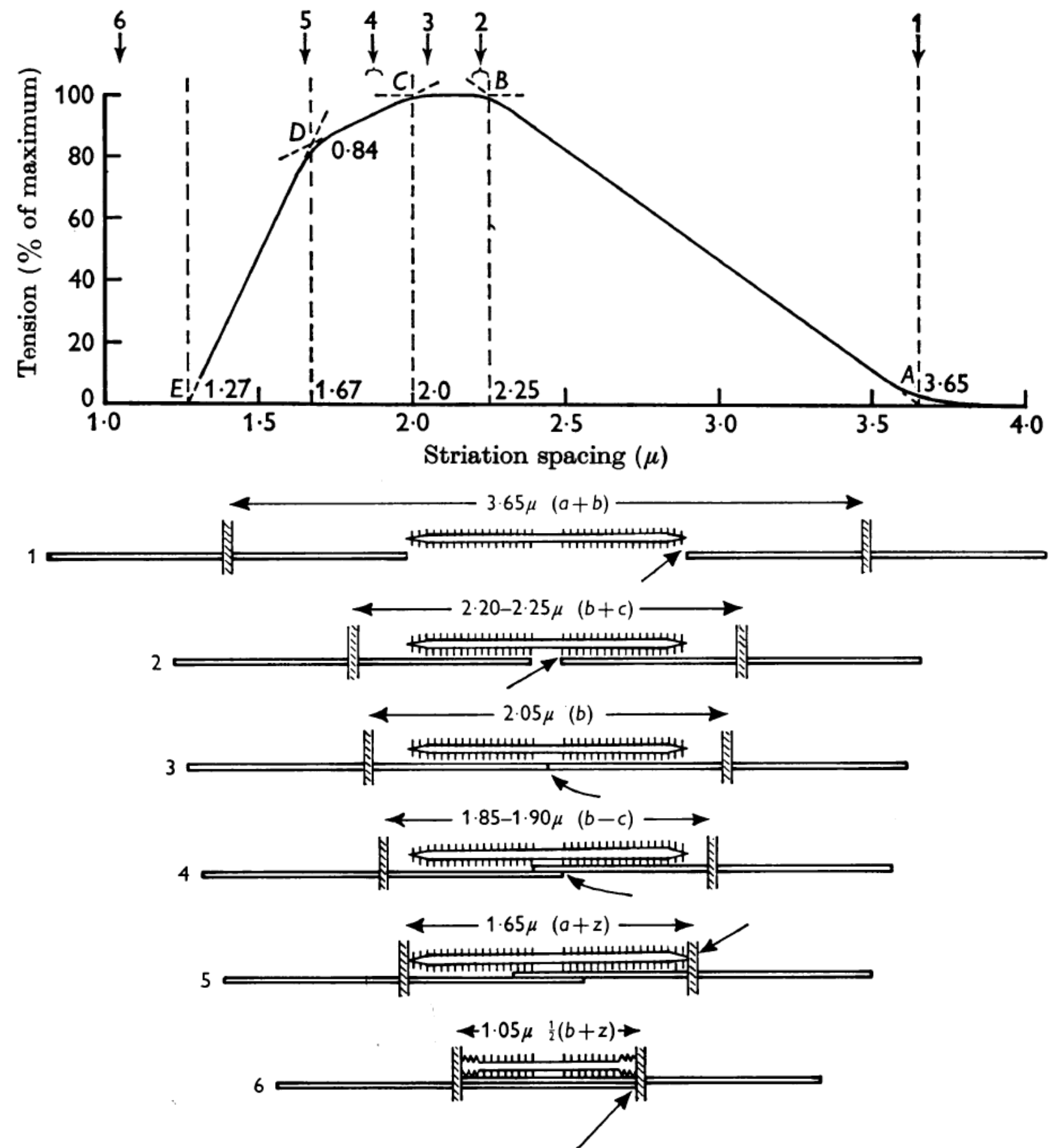

Figure 1.4 Schematic illustration relating the degree of filament overlap and corresponding force-length relationship.

Taken with permission from Gordon, Huxley, \& Julian, (1966). Force-length relationship of muscle (top), and the varying degrees of filament overlap that correspond to the key areas of the force-length curve (bottom). 


\subsubsection{How Velocity of Shortening Impacts Muscle Force Production}

The speed of contraction, or the shortening velocity of the muscle refers to how fast the muscle is shortening. One of the fundamental properties of muscle is the so-called "forcevelocity relationship," which shows that with increasing shortening speed, a muscle will not be able to produce as much force. The mechanism behind this relationship is related to several factors at the cross-bridge level. Firstly, the amount of force produced by each individual crossbridge decreases at high shortening velocities, and secondly, fewer cross-bridges are able to form as shortening velocities are increased (Piazzesi et al., 2007). This second mechanism has to do with the fact that myosin heads take some amount of time to attach to actin. When muscles shorten at very fast speeds, the actin and myosin filaments are sliding past one another so fast that the probability of filament interaction is decreased, reducing the number of cross-bridges formed, and effectively decreases the amount of force the muscle is capable of producing. Finally, at very high velocities, some proportion of the cross-bridges may not detach in time before they are actually resisting the shortening force, effectively decreasing the net shortening force (Huxley, 1957). In short, higher speeds of shortening will result in less muscle force via a combination of reduced force per cross-bridge, the potential for a "braking" force from undetached cross-bridges, and a reduced number of attached cross-bridges (see Figure 1.5). 


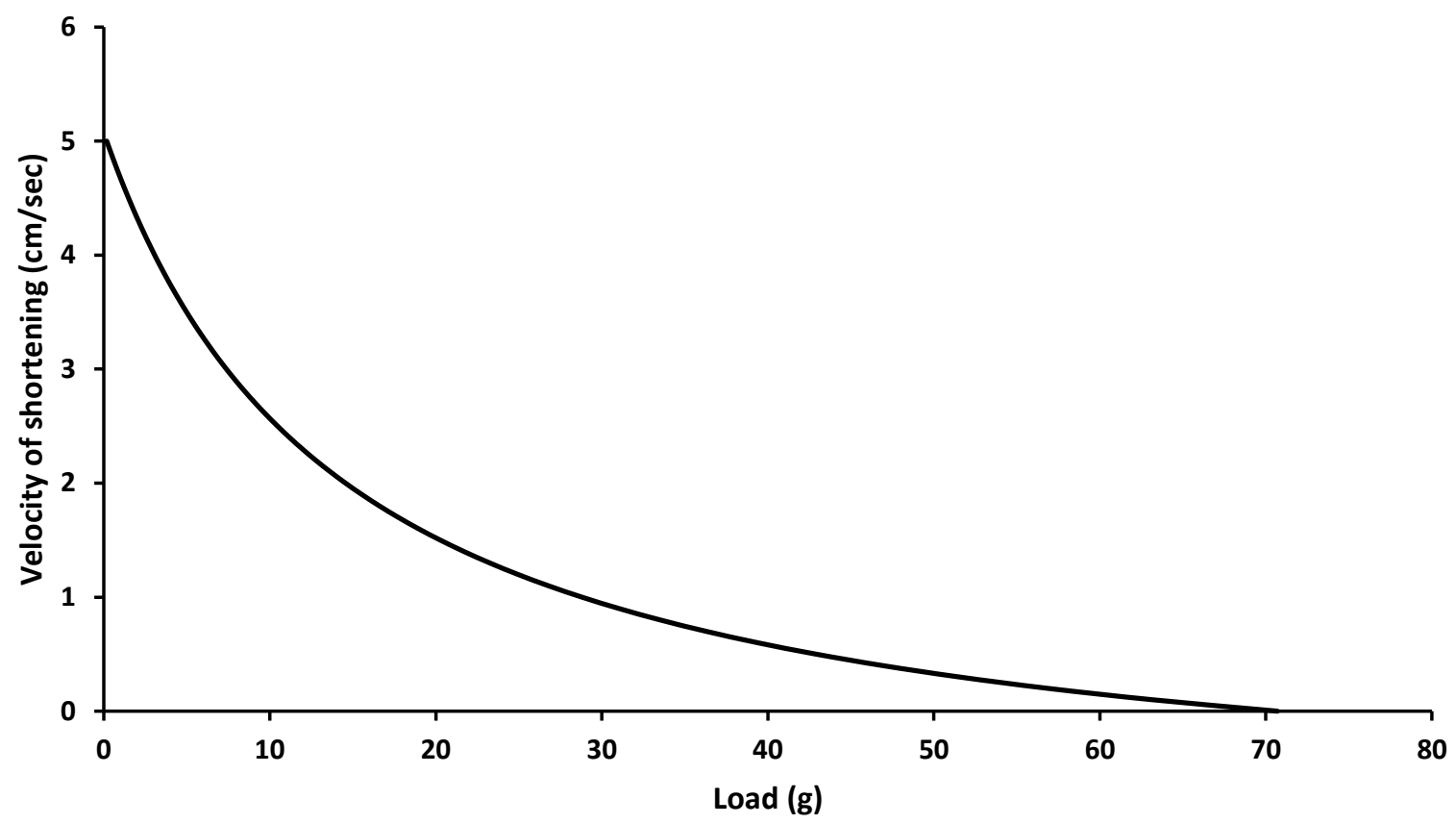

Figure 1.5 Force-velocity relationship of frog sartorius.

Based on data from Hill (1938). Note that as the load is increased, the velocity of shortening decreases.

It is important to note here that the force values in Figure 1.5 above are instantaneous data points, meaning that the force reached that level while the muscle was moving at that velocity. During truly isometric contractions, the muscle would not be shortening and thus the peak force will be attainted at a point of zero velocity. Of interest, however, is if there may be an "after-effect" of sorts due to the shortening velocity before peak force is attained at an isometric endpoint for brief contractions such as twitches. Conceivably, this may be due to the interaction between shortening velocity and calcium levels in the muscle, both impacting the relative rates of cross-bridge attachment and detachment.

As described earlier, after an action potential reaches the muscle cell, $\mathrm{Ca}^{2+}$ will be released from the sarcoplasmic reticulum and bind to troponin, allowing cross-bridges to form and muscle force production to occur. As $\mathrm{Ca}^{2+}$ is sequestered back into the sarcoplasmic 
reticulum, intracellular $\mathrm{Ca}^{2+}$ levels will begin to fall, $\mathrm{Ca}^{2+}$ will dissociate from troponin, and force production will decline. Parvalbumin, a $\mathrm{Ca}^{2+}$ buffering protein within the muscle cell, aids in this relaxation process by binding to the free $\mathrm{Ca}^{2+}$, and thus preventing it from binding to troponin (Rall, 1996). During twitch contractions, the rise in intracellular $\mathrm{Ca}^{2+}$ happens very rapidly, and begins to decline soon after force production even begins to rise (see Figure 1.6). Since intracellular $\mathrm{Ca}^{2+}$ levels are declining for the majority of the contraction, force may begin to fall based on the time course of the $\mathrm{Ca}^{2+}$ sequestration back into the sarcoplasmic reticulum, coming to a very low concentration before any true "isometric" point is met. If the shortening velocity is higher during this rise in force, this may decrease the number of cross bridges that are able to form before $\mathrm{Ca}^{2+}$ levels fall and force begins to decline, decreasing the level of peak force attained. While speculative, this notion will be elaborated on later, as it may affect the interpretation of our experimental findings. 


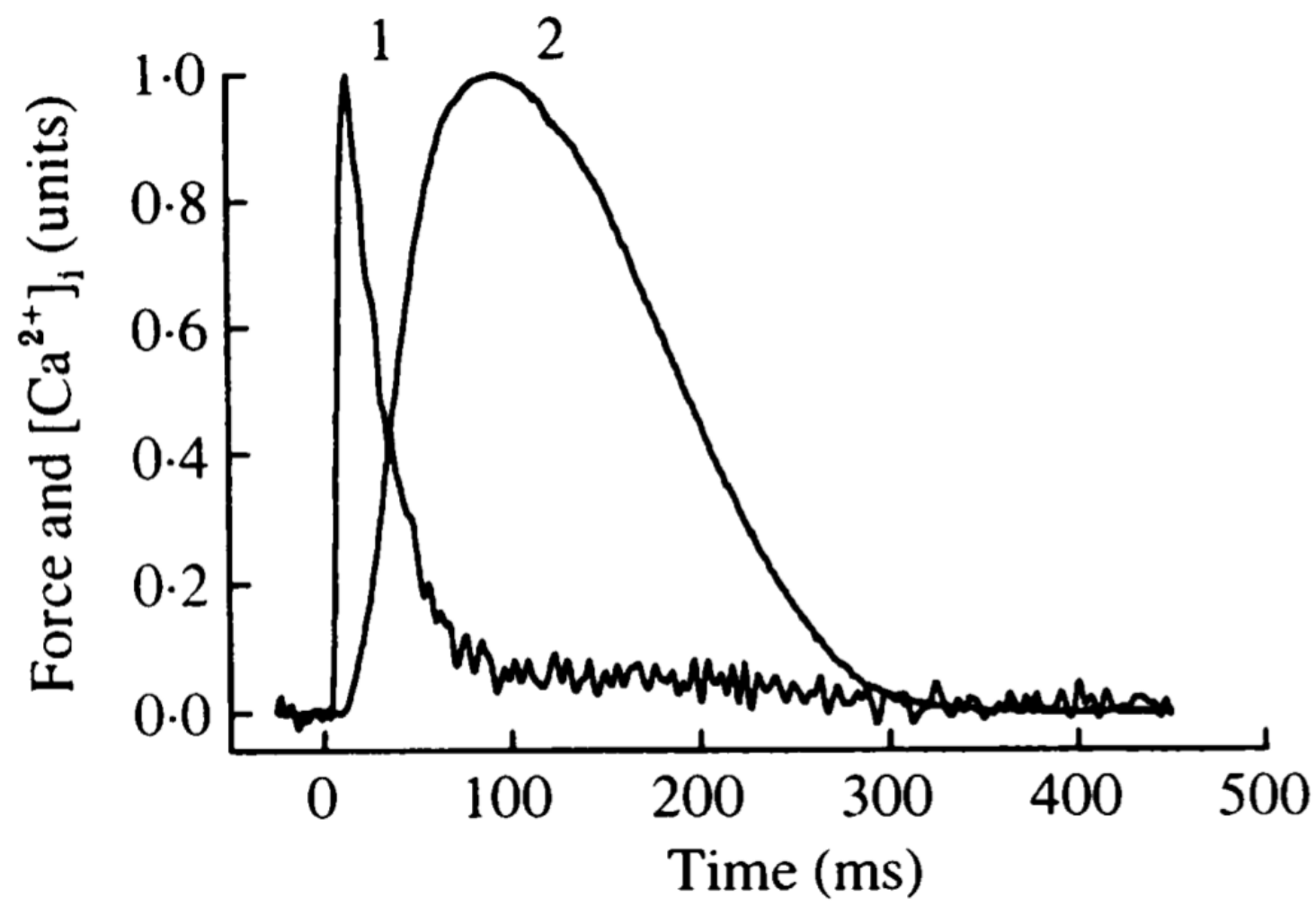

Figure 1.6 Intracellular $\mathrm{Ca}^{2+}$ transient and force during a twitch in frog muscle.

Taken with permission from Sun, Lou, \& Edman (1996). Curves are normalized relative to their peak amplitudes. The $\mathrm{Ca}^{2+}$ transient (curve 1) rises quickly after the stimulation, and begins to decline soon after the force (curve 2) begins to rise.

\subsection{Summary}

Muscles produce force in response to an activation of motor nerves and neuromuscular transmission; action potentials on muscle membranes lead to the release of calcium from the SR, which then binds to troponin, exposing the myosin binding sites on the actin filament via the movement of tropomyosin. This allows cross-bridges to form and force to be produced. When the excitation ceases, calcium is re-sequestered back into the SR causing calcium to then dissociate from troponin, covering back up the myosin binding sites on the actin molecule, and subsequently leading to a decrease in force as cross-bridges disengage. Described above are three key factors relating to muscle force production, namely the level of activation, the velocity of 
shortening, and the relative muscle length. All of these phenomena can be readily explained by the sliding filament theory and the cross-bridge theory of muscular contraction, based on the fact that force is proportional to the number of cross-bridges that can participate in force generation and factors that change the force per cross-bridge. What is to follow is a brief overview of how two of these characteristics - the level of activation and the length of the muscle - interact with one another in the production of muscle force, as well as an introduction to a separate determinant of muscle force that cannot be explained by the above theories: shortening-induced force depression. 


\section{The Determinants of the Optimum Length of Muscle}

\subsection{Measuring the Optimum length: Absolute length, Relative Length, and the Potential for Variances in the Force-Length Relationship}

In absolute terms, the force-length relationship chiefly depends on the length of the thick and thin filaments within a sarcomere, and these lengths happen to be dependent on the species of animal being looked at (Burkholder \& Lieber, 2001). Figure 1.4 shows a constructed forcelength relationship using frog muscle, with the corresponding sarcomere lengths (in $\mu \mathrm{m}$ ) along the bottom. These sarcomere lengths would be slightly different than what we might see with rats or humans, for example, due to the variance in length of the myofilaments. Specifically, both humans and frogs are known to have thick filament lengths of $1.60 \mu \mathrm{m}$, however frog thin filaments are only $0.98 \mu \mathrm{m}$ in length, while the thin filaments of humans are $1.27 \mu \mathrm{m}$. Based on the structure of a sarcomere, zero filament overlap will occur at a sarcomere length corresponding to sum of the thick filament length, plus two thin filament lengths, plus the width of the Z-disk separating adjacent sarcomeres $(\sim 0.1 \mu \mathrm{m})$. For a frog, that is approximately equal to $1.60 \mu \mathrm{m}+0.98 \mu \mathrm{m}+0.98 \mu \mathrm{m}+0.1 \mu \mathrm{m}=3.64 \mu \mathrm{m}$, very close to the value of $3.65 \mu \mathrm{m}$ reported on Figure 1.5. Humans, on the other hand, will have zero myofilament overlap at a sarcomere length of approximately $4.24 \mu \mathrm{m}(1.60 \mu \mathrm{m}+1.27 \mu \mathrm{m}+1.27 \mu \mathrm{m}+0.1 \mu \mathrm{m})$. As seen in Figure 1.4 , there is a "bare zone" on the thick filament that does not contain any myosin heads capable of forming cross-bridges, which is $\sim 0.20 \mu \mathrm{m}$ in width. Thus, the plateau of the force-length curve will correspond to a length ranging from approximately the length of two thin filaments on the short end, to that plus the width of this bare zone and the Z-line on the long end (see sarcomere illustrations 2 and 3 on Figure 1.4). From the given numbers above, this will be $\sim 2.05-2.25 \mu \mathrm{m}$ in frogs, but closer to $2.64 \mu \mathrm{m}-2.84 \mu \mathrm{m}$ in humans (Burkholder \& Lieber, 2001; Vaz, De La 
Rocha Freitas, Leonard, \& Herzog, 2012). Due to these discrepancies in absolute thin filament length amongst different species, it is often more convenient to make comparisons expressing the relative length of the muscle rather than the absolute length. In this case, the optimum length is assigned the value of 1 , or $100 \%$, and all lengths shorter and longer than the optimum will be expressed relative to this value. For example, if we assume the frog muscle from Figure 1.4 had an optimum length of $\sim 2.10 \mu \mathrm{m}$, then no filament overlap, and thus no force production, should occur at $\sim 3.6 \mu \mathrm{m}$, which is approximately 1.7 times, or $170 \%$ of the optimum length. This method of expressing length changes relative to the optimum length will be used from here on. It is assumed that a proportional change in muscle or fascicle length corresponds to a similar change in sarcomere length.

In vivo, the force-length relationship is often measured as a torque-angle relationship, as muscles attach to bones and produce rotation about joints. This relationship describes the amount of torque that can be produced as a function of joint angle, which in this circumstance is dependent both on the degree of filament overlap in the muscle, as well as the moment arm of the muscle (which may vary over a joint's range of motion). It is interesting to note that the in vivo shape of the torque-angle relationship may change with training, potentially due to the insertion or deletion of sarcomeres in series. In this regard, it has been shown that cyclists and runners display distinctly different moment-length relationships of the rectus femoris muscle, whereby runners could produce more torque at longer muscle lengths, while cyclists could produce more torque at shorter muscle lengths. While it is difficult to ascertain the precise reason for this observation and whether it was due to training or genetic factors, it may be due to adaptations caused by the hip positions they assume for training (Walter Herzog, Guimaraes, Anton, \& Carter-Erdman, 1991). In vivo, the muscle-tendon-unit (MTU) length is essentially 
fixed by the position of the bones and muscle attachments. Assuming a muscle operates near its optimum length, then at a given MTU length (or joint angle), the insertion of additional sarcomeres in-series would function to make the length of each sarcomere relatively shorter, compressing them towards the ascending limb of the force-length relationship. Now, the optimum joint angle would be pushed to slightly larger angles, corresponding to more optimal degree of myofilament overlap within the sarcomeres. The deletion of sarcomeres in series would do just the opposite; the sarcomeres would be stretched towards the descending limb, and thus the optimal joint angle would be pushed to smaller angles where myofilament overlap would be greater.

While a straightforward concept, we can see that some considerations need to be made when discussing the force-length relationship to ensure that there is no unnecessary ambiguity. Inter-species differences in myofilament lengths, different means of expressing muscle lengths (relative or absolute), and different experimental conditions in which the force-length relationship is being studied all have the potential to cause some confusion. Regarding different experimental conditions, it may be appropriate here to note some of the common inconsistencies in experimental protocols that may influence the reported findings. First is the discrepancy between an isometric contraction and a fixed-end contraction. An isometric muscle contraction, as one might discern from its name, is one in which the length of the muscle remains constant. Herein lies a very important distinction, however; a contraction in which the total MTU length remains constant may not be truly isometric at the level of the sarcomere. Any connective tissue (e.g. tendon) or other material used to connect the end of a muscle to the force measuring device may be compliant, allowing the actual muscle length to shorten upon activation, even though the MTU length may not change. The definitions used here differentiate true isometric contractions, 
in which the actual muscle fibres do not change length, from fixed-end contractions, in which the MTU length remains constant.

Related to this distinction of contraction type is the distinction in the method of measuring muscle length per se. Depending on the experimental setup used, muscle length may be measured by the sarcomere length, by the muscle fibre length, by the muscle fascicle length, or even by the MTU length. Again, this is an important distinction, because if the muscle length is taken to be the MTU length during a fixed-end contraction, or if muscle shortening during a contraction is not taken into account, then the actual muscle or sarcomere length inferred may be inaccurate. Indeed, this has been found to be an issue in previous reports. For example, Rack and Westbury (1969), in their landmark paper to be discussed in more detail shortly, found that the optimum sarcomere length of the cat soleus was $\sim 2.8-3.0 \mu \mathrm{m}$. Based on the known actin and myosin filament lengths in cats, this seemed to be an overestimation by about $0.5 \mu \mathrm{m}$. To tackle this discrepancy, Vaz, De La Rocha Freitas, Leonard, \& Herzog (2012) obtained the force-length relationship of the cat soleus using post mortem laser diffraction to measure sarcomere length, and found the optimum length to be $\sim 2.0-2.4 \mu \mathrm{m}$, closer to what would be expected based on the lengths of the myofilaments. The discrepancy here arose because Rack and Westbury did not in fact measure sarcomere length during their contractions, but only estimated it from passive relationships between sarcomere length and joint angles. Vaz et al (2012) went on to state that "Rack and Westbury measured the force-length properties of the cat soleus and measured sarcomere lengths following animal sacrifice. They assumed that the fibre length, and thus the average sarcomere length, was directly proportional to muscle length, thereby ignoring the possible shortening of fibres during force production in fixed end contractions." 
This illustrates the importance of defining what exactly is being measured, and accounting for any changes in what is being measured that may occur during a muscle contraction. This will be discussed again later, but first we will introduce another phenomenon relating to the force-length relationship, the length-dependence of activation.

\subsection{A Brief History of the Length Dependence of Activation}

As we have been discussing, the force-length relationship is a fundamental property of skeletal muscle, and has been known for over a century. First described by Blix (1891), it was then further explored by Evans and Hill (1914), Doi (1920, 1921), and Ramsey and Street (1941), among others. Suffice to say, this property has received much attention since the first experiments in the $19^{\text {th }}$ century, although it wasn't until Gordon, Huxley and Julian (1966) used the sliding filament theory proposed by A.F. Huxley \& Niedergerke (1954) and H. Huxley \& Hanson, (1954) and the cross-bridge theory (A.F. Huxley 1957) that the mechanism of the forcelength relationship was detailed (see Figure 1.4). Prior to this, there was very little discussion of exactly why this phenomenon existed, possibly because we knew little about how muscles contracted.

The force-length relationship describes the amount of isometric force a muscle can produce as a function of its length, and is typically studied using maximal, artificially stimulated contractions. Essentially, this relationship is due to the degree of overlap between the myosin and actin filaments; optimal overlap will allow the muscle to produce the most force, and lengths that are shorter or longer than this optimal length will produce less force. Rassier, MacIntosh and Herzog (1999) clarified the "force-length relationship" as that in which maximal isometric contractions were used, and differentiated this from the "length dependence of force," in which the contractions across the length range were submaximal. 
Rack and Westbury (1969) were some of the first to formally study this length dependence of force, by not only investigating the effect of length on the resultant force output, but the stimulus rate as well. They did this by artificially stimulating cat soleus at different muscle lengths and different frequencies of stimulation, and showed that at lower stimulation frequencies, the muscle length that produced the most active force was longer than that which produced the most active force at higher stimulus rates. They attributed this change to the notion that, "at short lengths the process of activation may function less effectively, so that each action potential gives a smaller, and perhaps briefer activation of the contractile machinery" (Rack \& Westbury, 1969). This phenomenon is referred to as the length dependence of activation and describes how a muscle's optimum length will be longer at submaximal activation levels than it is when maximally activated. The mechanism for length dependent activation has been further explored, overturning the initial explanation offered by Rack \& Westbury.

\subsection{Mechanisms of LDA in skeletal muscle}

\subsubsection{The Effects of $\mathrm{Ca}^{2+}$ Sensitivity on Activation-Dependent Shifts in Optimum Length: The Case for Proximity of Myofilaments}

The most accepted physiological explanation for the shift in optimum length with submaximal activation is that there is a length-dependent increase in $\mathrm{Ca}^{2+}$ sensitivity, due to the myofilament lattice space decreasing as the muscle is stretched. There is strong evidence for this assertion. To begin exploring the evidence, we must go back far beyond Rack and Westbury's pioneering study, to an experiment done by Doi in 1920. Doi tested the force output for twitch contractions of a frog muscle as a function of its initial length at two different temperatures. $\mathrm{He}$ found that at $15^{\circ} \mathrm{C}$, the muscle not only produced less peak force, but its optimal length was shifted to longer lengths compared to $5^{\circ} \mathrm{C}$ (see Figure 2.1 below). 


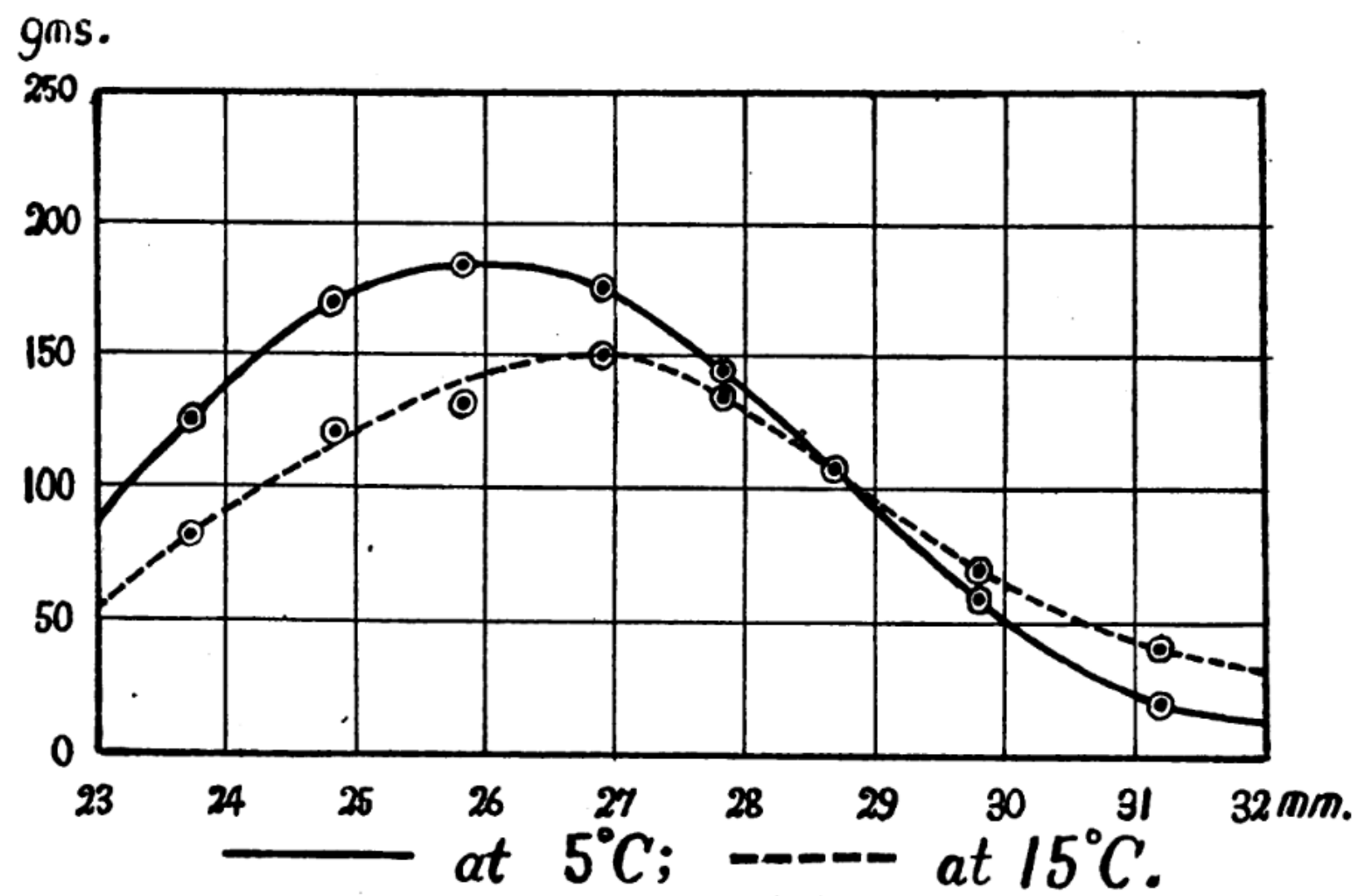

Figure 2.1 The influence of temperature on the force-length relationship. Taken with permission from Doi (1920). Shown are force-length curves of frog gastrocnemius muscle at $15^{\circ} \mathrm{C}$ (dashed line) and at $5^{\circ} \mathrm{C}$ (solid line). Note the decreased peak force and longer optimum length at $15^{\circ} \mathrm{C}$ than at $5^{\circ} \mathrm{C}$, likely explained by the effects of temperature and length on the $\left[\mathrm{Ca}^{2+}\right]$ and $\mathrm{Ca}^{2+}$ sensitivity.

The fact that Doi saw an increase in peak force at lower temperatures is interesting, since it is known that force per cross bridge increases with temperature. This discrepancy probably relates somewhat to the fact that the twitch:tetanus ratio is higher at cold temperature, and also to some contractile characteristics that are unique to certain species of frogs. Specifically, Kössler, Lange, \& Küchler, (1987) reported an increase in twitch tension and decrease in tetanic tension with decreasing temperature in frog muscle, suggesting that there was some sort of cold-induced twitch potentiation going on that both increased $\mathrm{Ca}^{2+}$ release and slowed $\mathrm{Ca}^{2+}$ reuptake of twitches at cooler temperatures, a notion that was supported by Caputo, Gerday, Lopez, Taylor, \& Bolaños (1998). However, in Doi's experiment, the fact that the difference in force was 
greater at shorter lengths compared to longer lengths gives us some indication that the effect may also have been due to the effects that temperature and sarcomere length have on $\mathrm{Ca}^{2+}$ sensitivity. To explain the shift in optimum length that Doi reported, we must first look at the impact of temperature on $\mathrm{Ca}^{2+}$ sensitivity. Stephenson and Williams (1985) showed that an increase in temperature resulted in a decrease in $\mathrm{Ca}^{2+}$ sensitivity in skinned fibers of the rat and toad (see Figure 2.2 below).

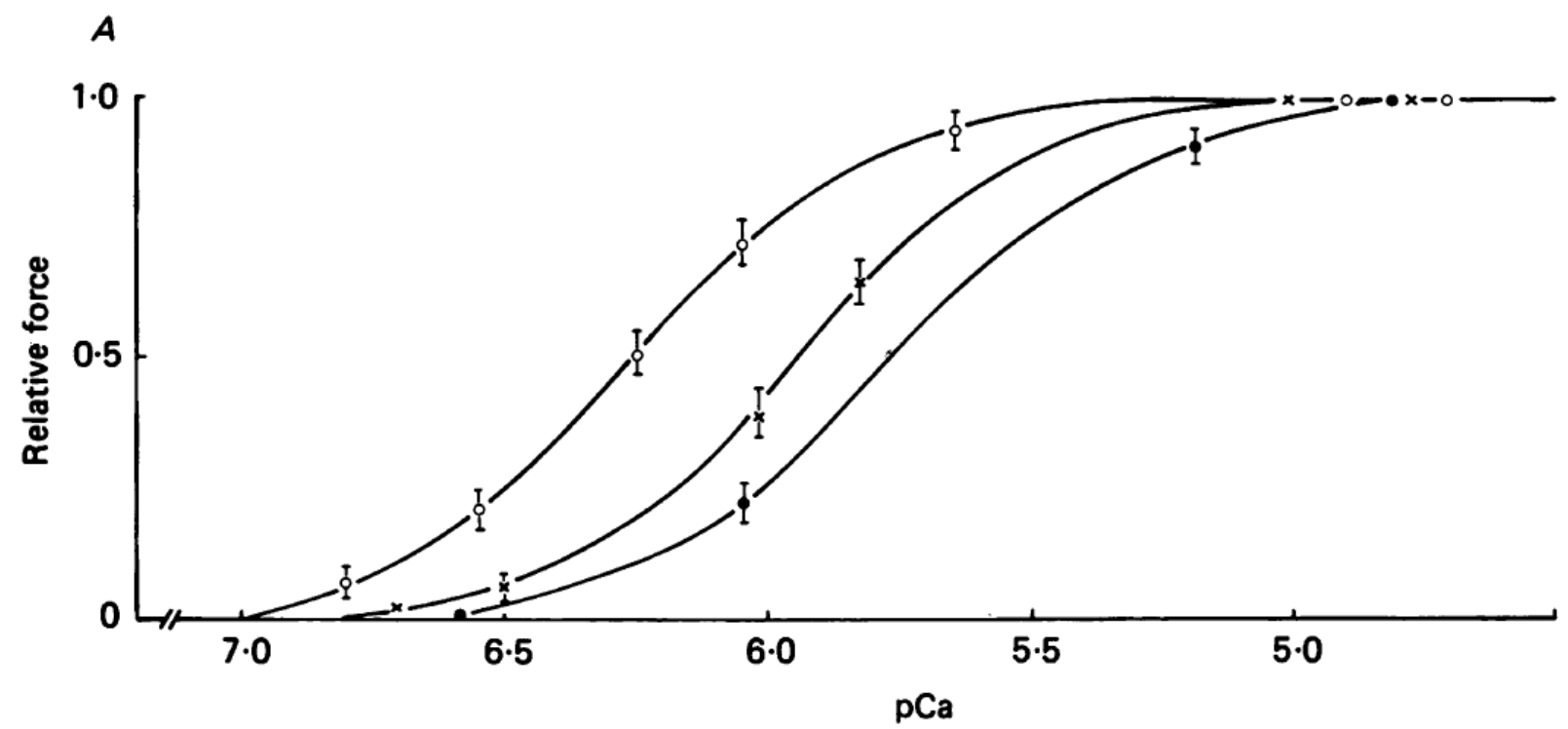

\section{Figure 2.2 The effect of temperature on $\mathrm{Ca}^{2+}$ sensitivity.}

Taken with permission from Stephenson and Williams (1985). Rat soleus skinned fibre forcepCa relationships at temperatures of $35^{\circ} \mathrm{C}$ (closed circles), $22^{\circ} \mathrm{C}(\mathrm{x})$, and $5^{\circ} \mathrm{C}$ (open circles). Note that the curves are shifted leftwards $\left(\mathrm{Ca}^{2+}\right.$ sensitivity increases) as temperature decreases. Calcium concentration is expressed as a negative $\log$ where 6.0 is $10^{-6}$ moles per litre.

The reason for this change in $\mathrm{Ca}^{2+}$ sensitivity can be explained by $\mathrm{Xu}$, Offer, $\mathrm{Gu}$, White, and $\mathrm{Yu}$ (2003), who used X-ray diffraction to look at the conformation of the myosin heads at low or high temperature. They showed that at high temperatures, the myosin heads in the relaxed muscle are in a very "ordered" state while at low temperatures, the myosin head are much more "disordered." The interpretation of this apparent disorder is that it suggests a larger proportion of the myosin heads are extending out from the filament backbone, compared to the ordered state in 
which the majority of the myosin heads are situated close to the backbone. Presumably, this would create a situation where at low temperatures, the distance between actin and the myosin head is small, which would have a positive effect on the probability of filament interaction and cross-bridge formation. At high temperatures, the myosin heads are positioned farther from the thin filaments, and thus there is a smaller probability of interaction at submaximal $\left[\mathrm{Ca}^{2+}\right]$.

In regard to the impact of sarcomere length, Endo (1972) found that in skinned muscle fibres, "at lower calcium concentrations, greater tension was developed at longer lengths, in spite of decreased magnitude of overlap between two kinds of filaments" (Endo, 1972). Similarly, Stephenson and Williams (1982) showed that the force-pCa curve of skinned fibres of the rat is shifted to the left when sarcomeres are stretched to longer lengths (see Figure 2.3 below). Endo (1972) postulated that this length dependent $\mathrm{Ca}^{2+}$ sensitivity may be due to "the smaller distance between thick and thin filaments at longer lengths [which] might facilitate the interaction between actin and myosin." A closer proximity between actin and myosin would tend to increase the probability of filament interaction, and thus provide an environment in which the likelihood of cross-bridge engagement was increased for a given submaximal $\left[\mathrm{Ca}^{2+}\right]$. See Figure 2.4 below for an illustration of this suggestion. 


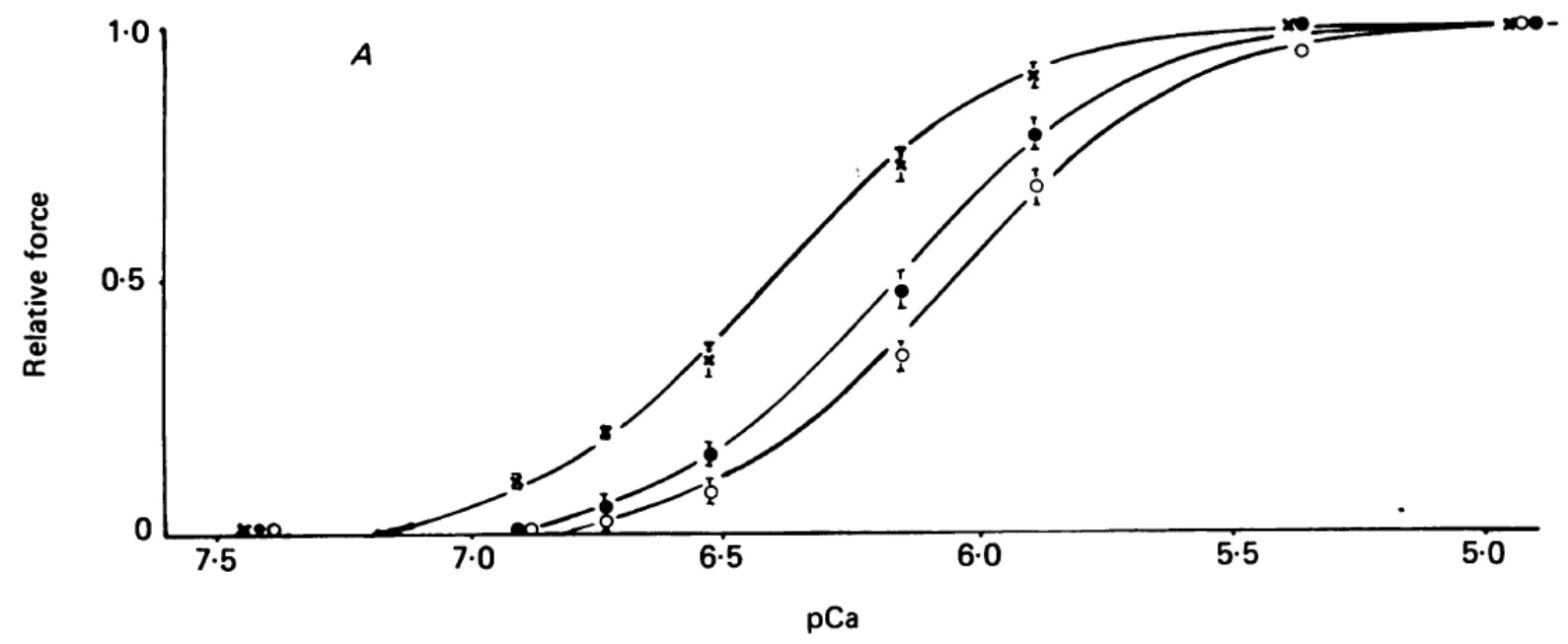

Figure 2.3 The effect of sarcomere length on $\mathrm{Ca}^{2+}$ sensitivity.

Taken with permission from Stephenson and Williams (1982). Rat soleus skinned fibre forcepCa relationships at sarcomere lengths of $2.76 \mu \mathrm{m}$ (open circles), $3.13 \mu \mathrm{m}$ (closed circles), and $3.60 \mu \mathrm{m}(\mathrm{x})$. Note that as sarcomeres are stretched to longer lengths, the force-pCa curve is shifted leftwards, meaning that more force is produced for a given concentration of $\mathrm{Ca}^{2+}$. Also note that at the highest $\left[\mathrm{Ca}^{2+}\right]$, there is no further change in force output, showing that this increase in $\mathrm{Ca}^{2+}$ sensitivity is only apparent at submaximal $\mathrm{Ca}^{2+}$ concentrations. Note that these forces have been normalized for the maximal force at a given length. Absolute active force will be less at long sarcomere lengths.

Taking both of these effects into account, Doi's results for twitch contractions can be explained; at a lower temperature, there is a higher $\mathrm{Ca}^{2+}$ sensitivity than at high temperatures, contributing to the larger peak force. At high temperatures, since the myosin head is situated farther away from the actin filaments, which reduces the likelihood of cross-bridge formation. Importantly, however, this greater distance between filaments is partly compensated for by the reduced distance when the muscle is stretched to longer lengths. At these longer lengths, the myosin heads are sufficiently close to the actin filaments such that there is very little difference in force production between low or high temperatures, as the probability of interaction must be equal. These two simultaneous effects, both influencing $\mathrm{Ca}^{2+}$ sensitivity by their impact on the proximity of the myosin head and actin, end up shaping the relationship between these two forcelength curves seen in Figure 2.1. 
For further evidence that changes in $\mathrm{Ca}^{2+}$ sensitivity are due to changes in myofilament spacing, we can look to the work of Stienen, Blangé, \& Treijtel (1985), who investigated the effects of both sarcomere length as well as dextran on $\mathrm{Ca}^{2+}$ sensitivity in skinned muscle fibres of the frog. Dextran exerts an osmotic pressure on the skinned muscle fibre, causing the myofilaments to move closer together as a result of fluid moving out of the myofibril. They found that, similar to Stephenson and Williams (1982), $\mathrm{Ca}^{2+}$ sensitivity increased at longer sarcomere lengths. Additionally, they found that reducing the lattice spacing with dextran also increased $\mathrm{Ca}^{2+}$ sensitivity, but that this effect was only apparent at shorter sarcomere lengths. It is important to note that in skinned fibre experiments, temperatures are kept below physiological temperatures (in this particular case $5^{\circ} \mathrm{C}$ ). As we have seen, decreased temperature will increase $\mathrm{Ca}^{2+}$ sensitivity on its own via changes in myosin head positioning, and this fact may impact the relative effects reported. Nonetheless, this interaction basically mirrors the results of Doi (1920), in the sense that both dextran, as well as reduced temperatures, will increase $\mathrm{Ca}^{2+}$ sensitivity by bringing the myofilaments close together, but this effect is negated at longer lengths since at these lengths the lattice spacing is already reduced.

To this point, the main take-home message is that shifts in optimum length between different stimulation conditions can be largely attributed to changes in $\mathrm{Ca}^{2+}$ sensitivity, and this change in $\mathrm{Ca}^{2+}$ sensitivity can largely be attributed to the proximity of myosin heads with actin filaments, affecting the probability of cross-bridge attachment at a given $\left[\mathrm{Ca}^{2+}\right]$. So far, we have explored the effects of temperature and sarcomere length on $\mathrm{Ca}^{2+}$ sensitivity. To explain the results seen by Rack and Westbury (1969) we must touch on the effects of stimulation frequency and free myoplasmic $\left[\mathrm{Ca}^{2+}\right]$. At lower stimulation frequencies, less $\mathrm{Ca}^{2+}$ is released by the sarcoplasmic reticulum, and thus there are fewer binding sites for myosin to interact with 
compared to maximally activated muscle. As stated above, for a given submaximal $\left[\mathrm{Ca}^{2+}\right]$, this probability of attachment increases as sarcomere length increases, effectively "outweighing" the reduced amount of filament overlap, leading to a shift in optimum length to longer lengths when $\left[\mathrm{Ca}^{2+}\right]$ is low. When $\left[\mathrm{Ca}^{2+}\right]$ is maximal, the probability of interaction between actin and myosin is already high since $\mathrm{Ca}^{2+}$ on its own can promote the movement of the myosin head away from the filament backbone (Podlubnaya, Kạkol, Moczarska, Stẹpkowski, \& Udaltsov, 1999), such that any small changes in $\mathrm{Ca}^{2+}$ sensitivity due to sarcomere length changes have little effect, and the optimum length will correspond to optimal filament overlap.

With these distinct, but related examples, we can glean some insight into the likely mechanisms behind this shift in optimum length in skeletal muscle. Doi (1921) reported a rightward shift in optimum length with increased temperature. This was likely due to the combined effects of an increased $\mathrm{Ca}^{2+}$ release at lower temperatures, an increased $\mathrm{Ca}^{2+}$ sensitivity at lower temperatures, as well as an increased $\mathrm{Ca}^{2+}$ sensitivity at longer muscle lengths. Rack and Westbury (1969) reported a rightward shift in optimum length at a given temperature with decreasing activation level. In both cases, $\mathrm{Ca}^{2+}$ release was submaximal, and in both cases, the relative proximity of actin and myosin filaments over the range of muscle lengths tested can help explain the relative shifts reported. To make the case that these shifts are due to alterations in $\mathrm{Ca}^{2+}$ sensitivity, and not some effect that changes in length have on $\mathrm{Ca}^{2+}$ release, it would be prudent to recognize that Balnave and Allen (1996) showed that changes in muscle length did not affect $\left[\mathrm{Ca}^{2+}\right]$ for a given frequency of stimulation in mouse fibres. Nevertheless, there may be other factors at play, which we will expound upon next. 

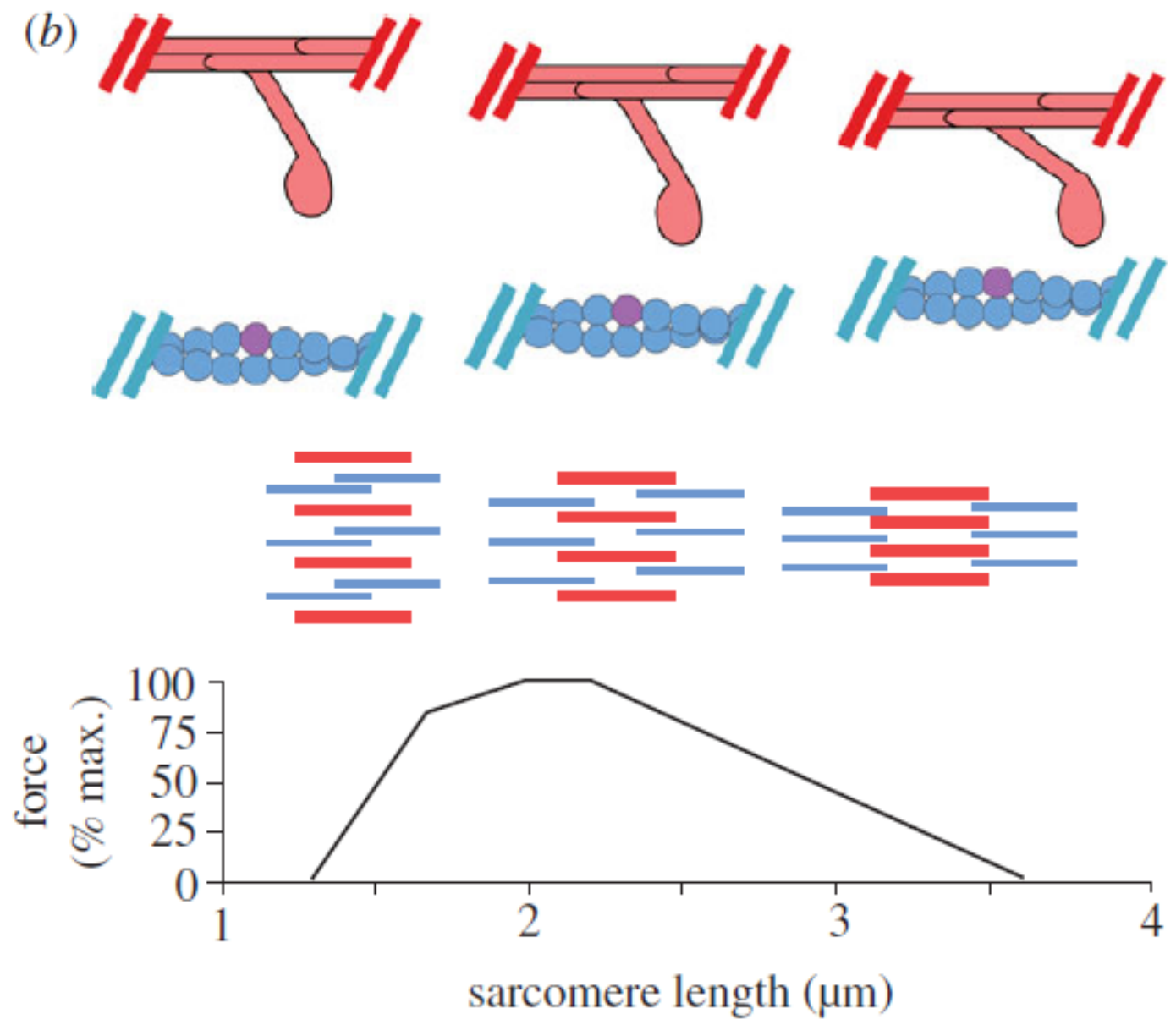

Figure 2.4 Schematic illustration of changes in filament proximity based on changes in sarcomere length, and the corresponding force-length relationship. Taken with permission from Williams, Salcedo, Irving, Regnier, \& Daniel (2013). Shown are illustrations of filament overlap and myosin head proximity on the ascending limb (left), plateau region (middle), and descending limb (right). As the muscle is stretched to longer lengths, the degree of filament overlap decreases, but the filament proximity increases.

\subsection{2 $\mathbf{C a}^{2+}$ Independent Effects on the Activation-Dependent Shift in Optimum Length}

While the length dependent increase in $\mathrm{Ca}^{2+}$ sensitivity is the most well-accepted theory of the length dependence of activation, it is not the only theory. To test the theory that $\mathrm{Ca}^{2+}$ sensitivity is the dominant factor, Holt and Azizi (2014) stimulated bullfrog plantaris muscle with single pulses as well as at a range of voltages at $100 \mathrm{~Hz}$, with the aim of determining if it was in fact increased $\mathrm{Ca}^{2+}$ sensitivity in the muscle, or the effect of absolute force, which was 
responsible for shifting the optimum length to longer lengths at submaximal activation levels. In a way, they were searching for a mechanical effect on LDA, separate from the physiological effect of $\mathrm{Ca}^{2+}$ sensitivity. The stimulation conditions they used were designed to create conditions in the muscle which had a high force output with a high $\left[\mathrm{Ca}^{2+}\right]$, a low force output with a low $\left[\mathrm{Ca}^{2+}\right]$, and a low force output with a high $\left[\mathrm{Ca}^{2+}\right]$. These combinations consisted of stimulating the nerve innervating the muscle with a high-frequency train of supramaximal pulses (high force/high $\left[\mathrm{Ca}^{2+}\right]$ ), a twitch contraction in which a single supramaximal pulse was applied (low force/low $\left[\mathrm{Ca}^{2+}\right]$ ), and by stimulating the nerve with a high frequency train but lower voltage than the first condition (low force/high $\left[\mathrm{Ca}^{2+}\right]$.) This last condition would maximally activate a small number of the motor units, based on the nerve axon's proximity to the stimulating electrode, and not activate deeper axons in the bundle. If LDA was in fact based on increased $\mathrm{Ca}^{2+}$ sensitivity, then one would predict that the optimum length would be the same for both conditions in which $\left[\mathrm{Ca}^{2+}\right]$ was high, as the key variable, $\left[\mathrm{Ca}^{2+}\right]$, was assumed to be similar. Remarkably, Holt and Azizi reported that optimum length increased in this low force condition, even when $\left[\mathrm{Ca}^{2+}\right]$ was assumed to be high, and concluded that, rather than solely a length dependent increase in $\mathrm{Ca}^{2+}$ sensitivity, perhaps it was just less-than-maximal force that impacted the shift in optimum length that they saw. They reported the following:

The force output of a muscle depends on both the intrinsic force production capacity of the contractile apparatus and the effectiveness of the force transmission system within muscle. The force transmission system of muscle is structurally complex, consisting of longitudinal pathways along myofilaments as well as lateral pathways across connective tissue elements. Some amount of internal work is likely needed to stretch and reorient the passive elements to allow for effective force transmission. This internal work will be 
relatively constant across activation levels. However, fewer cross-bridges are active at lower activation levels, so the constant requirement for internal work is likely to have a relatively greater effect. This internal work is, however, likely to vary with muscle length. Stretching the muscle to longer lengths may lower intramuscular compliance and decrease the internal work needed for effective force transmission. Hence, at low activation levels, where internal work may be more important, increasing muscle length beyond optimal actin-myosin overlap may ultimately result in greater force output owing to the reduction in internal work requirements.

The assertion that the optimum length is shifted to longer and longer lengths as the force output is decreased per se must be questioned, because in fact, the opposite findings have also been reported. MacNaughton, Campbell and MacIntosh (2007) investigated force-length profiles using twitch, double-pulse, and $50 \mathrm{~Hz}$ stimulation contractions, and found that the optimum length of the $50 \mathrm{~Hz}$ contraction occurred at a longer length than for double pulse, despite a higher force for the $50 \mathrm{~Hz}$ contractions. Considering that Holt and Azizi's argument revolves around the notion that low force outputs are the cause of the shift in optimum length, this finding suggests that their explanation is incomplete.

Additionally, a key concern with this report is the suggestion that the optimum length of contractions with $\sim 10 \%$ of maximal force were shifted to close to $70 \%$ longer than the maximal tetanic optimum length. A 70\% increase in sarcomere length should bring the muscle to a length where no filament overlap would be present (see section 2.1 for details on this), and thus no force production would be expected. Thus, it raises concern that their method of calculation or 
some other error may have affected their results, analogous to Vaz et al (2012) noting the incompatibility of Rack and Westbury's reports on sarcomere length.

Holt and Williams (2018) expanded on the 2014 paper, and attempted to explain what was seen by the phenomenon of shortening-induced force depression. They re-analyzed the data from 2014, and, knowing that the bullfrog plantaris tendon is quite compliant, they compared the relative shift in optimum length in this "compliant" condition to a separate set of experiments in which they used a bundle of fibres with no tendon attached to negate any muscle shortening upon activation. Interestingly, with less muscle shortening, they reported a substantially smaller shift in optimum length compared to the more compliant condition. For some reason, when the muscle was allowed to shorten during the contraction, the twitch optimum length was shifted significantly further to the right relative to the tetanic optimum length compared to an apparent isometric contraction. Curiously, they interpreted this result not as a greater rightward shift of the twitch optimum length, but a leftward shift of the tetanic optimum length when it was allowed to shorten during the contraction, causing the relative shift to be significantly greater in the high-compliance condition. They suggested that perhaps shortening-induced force depression was a factor in this greater relative shift.

\subsection{Shortening-Induced Force Depression and its Potential Role in Determining Skeletal Muscle Optimum Length}

\subsubsection{History and Mechanisms of Shortening-Induced Force Depression}

Shortening-induced force depression has been described since at least 1942, when Buchtall stimulated isolated fibres of the frog semitendinosus at varying lengths, and found that if the fibres were stimulated at a longer length and then shortened, they produced less force at the final length than if the fibres were stimulated isometrically at that same final length. This 
observation proved to be a curious one. As mentioned previously, the force-length relationship of muscle had been described since at least 1891 by Blix, and was quite well studied. As expounded upon in Section 1.4, it wasn't until Gordon, Huxley and Julian (1966) related this force-length relationship to the sliding filament theory of muscle contraction, however, that a working mechanism could describe the experimental results that people were seeing. Shortening-induced force depression, on the other hand, remains somewhat of a mystery. Since Buchtall's well detailed paper, there have been many more to look at this phenomenon of force depression, yet the mechanisms theorized have yet to be fully accepted as they were with the basic force-length relationship. The potential mechanisms to explain this phenomenon have included theories such as an accumulation of fatigue products, sarcomere non-uniformity, and a stress-induced inhibition of cross-bridges (Rassier and Herzog 2004). Because ATP consumption increases with more shortening, there is the potential for increased inorganic phosphate and hydrogen ion accumulation during shortening contractions, so this was suggested as a potential factor in this shortening-induced force depression (Granzier \& Pollack, 1989). However, the fact that force depression ceases once the muscle is quickly deactivated suggests that this hypothesis cannot explain the phenomenon, as these biproducts would not be expected to disappear immediately upon deactivation. Gross sarcomere length non-uniformity has been another attractive explanation for the depression of force after active shortening. The rationale here is that, upon active shortening, some proportion of the sarcomeres will shorten to a greater extent than average, to a point on the ascending limb of the force length curve where they are unable to produce as much force. Some other proportion of the sarcomeres will shorten to a lesser extent than expected, or even lengthen some, putting them further from the optimum length than would be expected based on the muscle length and estimated average sarcomere length. The net result is 
that, rather than all the sarcomeres being at an intermediate length near the optimum, some are shorter, and some are longer, decreasing the potential for active force production. One of the suppositions of this non-uniformity theory is that large sarcomere length non-uniformities theoretically should not be observed on the ascending limb of the force-length relationship. The idea behind this is that any length non-uniformity in this region would be expected to decrease upon activation, since the longer sarcomeres will be stronger, pulling the weaker shorter sarcomeres to longer lengths and essentially evening out the length distribution. With this premise in mind, one would not expect that force depression would be evident at these short lengths, yet it has been observed (Granzier \& Pollack, 1989), raising skepticism about the nonuniformity theory of force depression. With these points in mind, to date, it appears that the best explanation for shortening induced force depression is an actin strain-dependent inhibition of cross-bridge binding. In this case, the purported mechanism involves the actin filaments being actively strained upon activation, and distorting the regular position of myosin binding sites. This explanation fits with the finding that the amount of force depression seems to be related to the work done by the muscle. Work is the product of force and distance shortened; increased force has the potential to elongate the actin filaments to a greater extent, potentially distorting more myosin binding sites and reducing the probability of cross-bridge formation. An increased distance of shortening means that there will be a larger region of new filament overlap postshortening, whereby a larger proportion of the total filament overlap will have been "distorted" by the strain of the actin filaments, thus decreasing force to a greater extent compared to smaller shortening distances. As an example based on some of the work from Herzog, Leonard, \& Wu (2000), if two muscles produce an identical amount of force, but one muscle is shortened twice the distance of the other, it might have twice the amount of force depression due to the larger 
region of new filament overlap. Likewise, if two muscles are shortened the same distance, but one shortens with twice the force of the other, it might similarly have twice the amount of force depression due to the greater strain on the actin filaments. Related to what we learned earlier on the effects of shortening velocity on force, it is interesting to consider then that force depression is decreased at higher velocities (Meijer, Grootenboer, Koopman, \& Huijing, 1997), presumably due to the reduction in muscle force (and therefore actin strain). Taken together, active shortening when there are greater forces involved during the shortening, as well as greater shortening distances, will result in increased force depression compared to shortening smaller distances with smaller forces.

\subsubsection{Can Shortening-Induced Force Depression Impact Optimum Length?}

As mentioned earlier, Holt and Williams (2018) attempted to explain their results by stating that shortening-induced force depression may be apparent with more fibre shortening upon activation, and this force depression may impact the muscle length at which peak force occurs. While unproven, the purported mechanism here relates to the idea that, at longer starting lengths, and therefore less filament overlap, there will be less potential cross-bridges formed immediately upon activation, and so when it shortens, there will be a large region of "new" filament overlap that will be inhibited by strain-dependent force depression. On the other hand, when the muscle begins at shorter lengths where there is greater filament overlap to begin with, more cross-bridges can form immediately upon activation, and upon shortening, the "new" region of filament overlap is smaller, lending itself to relatively less force depression. Roughly in line with this, Granzier and Pollack (1989) noted the largest reductions in force were when the muscle stopped shortening at the optimum length, and when it shortened to lengths shorter than this, the relative degree of force depression was smaller. Relatively greater force depression at 
longer lengths conceivably could lead to an apparent leftward shift in the optimum length, in a force-dependent manner (e.g. higher force contractions would be depressed more than lower force contractions due to greater shortening, and an increased strain of the actin filaments). In agreement with this thought, Meijer, Grootenboer, Koopman, \& Huijing (1996) reported a length dependency of the ratio of the relative reduction in force output to the change in length in rat gastrocnemius muscle (see Figure 2.5 below).

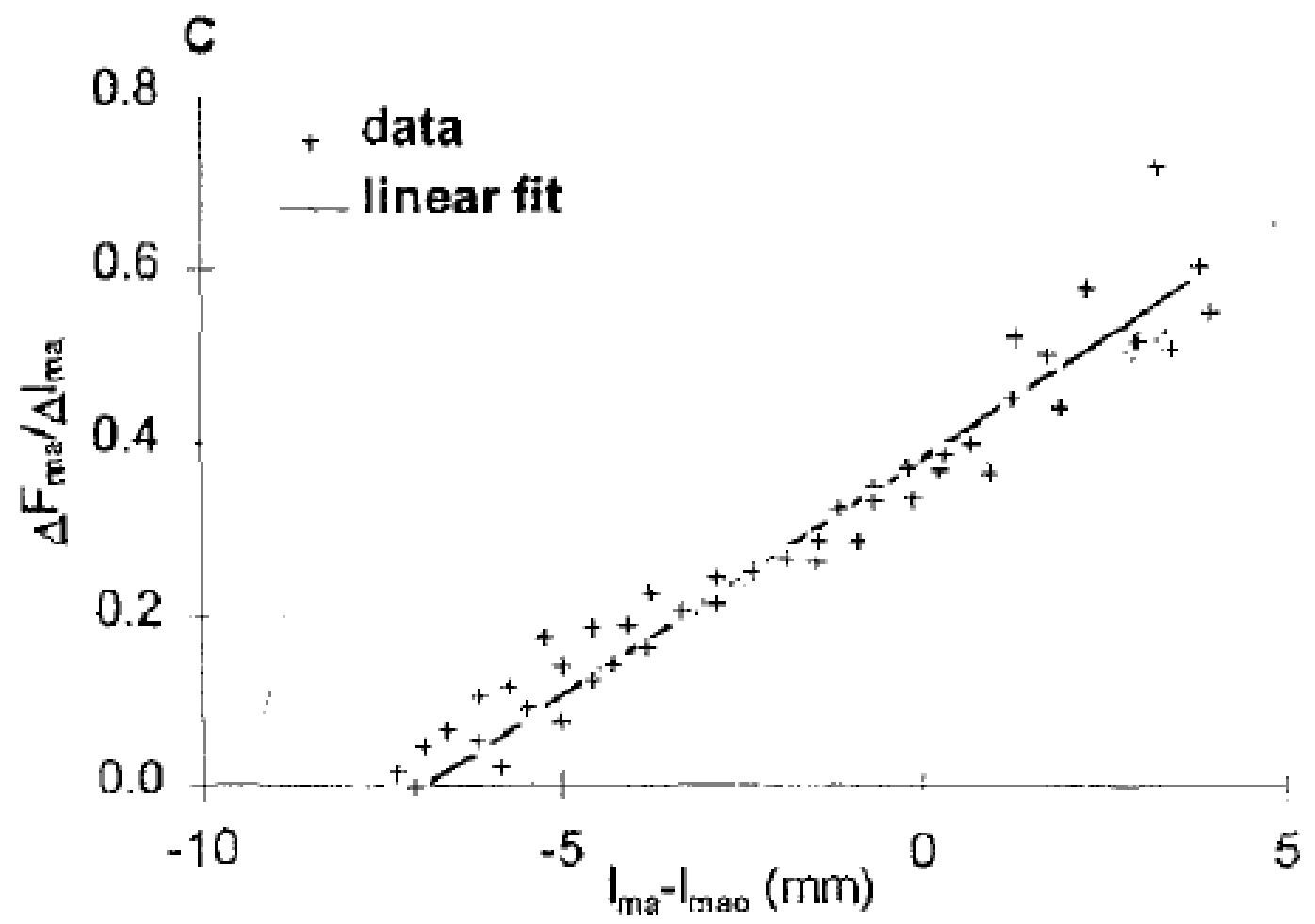

Figure 2.5 The length-dependence of force depression.

Taken with permission from Meijer et al (1996). They reported that the ratio of the reduction in force to the change in length was greater at longer muscle starting lengths. That is to say that, for a given amount of shortening, the relative reduction in force is greater when the muscle has been stretched to longer lengths. This may have the potential to effectively decrease the apparent optimum length. () 1996 IEEE.

In other words, for a given change in muscle length, the amount of force depression is greater at longer muscle lengths than shorter muscle lengths. Again, this may have the potential 
to cause an apparent leftward shift in the optimum length if force output after shortening is depressed to a greater extent at longer muscle lengths. The impact that force depression may have on the optimum length has not been extensively studied. However Meijer, Grootenboer, Koopman, \& Huijing (1997) did subsequently look at the effect of various shortening histories on the force-length relationship in rat medial gastrocnemius. They obtained force-length relationships in a fully isometric condition as well as with a variety of shortening lengths and velocities to determine any effect that shortening-induced force depression had on the postshortening force-length relationship (see Figure 2.6 below). They found that the most forcedepressed contractions had an optimum length shifted very slightly to the left compared to the contractions that were relatively less depressed. In this setup, however, the recorded muscle length was not the muscle fascicle or muscle fibre length, but the whole MTU length. This is significant, because with the tendon still attached, there would potentially be some series compliance and therefore some fibre shortening upon activation, however small. Lower force contractions would stretch the tendon to a lesser extent, and so for a given MTU length, a lower force contraction would have a longer muscle fibre length. Thus, the fact that they saw a slight leftward shift in optimum $M T U$ length only for the lowest force conditions may be explained by the fact that, since the actual muscle fibres will be longer at a given MTU length due to less tendon stretching, a shorter MTU length may still correspond to an equivalent muscle fibre length. Nevertheless, the experimental setup that Meijer et al used here would minimize the amount of muscle shortening, so whether this apparent shift in optimum length due to shortening-induced force depression is a real phenomenon or if it is an artifact of the method of measurement, it clearly needs further study. 

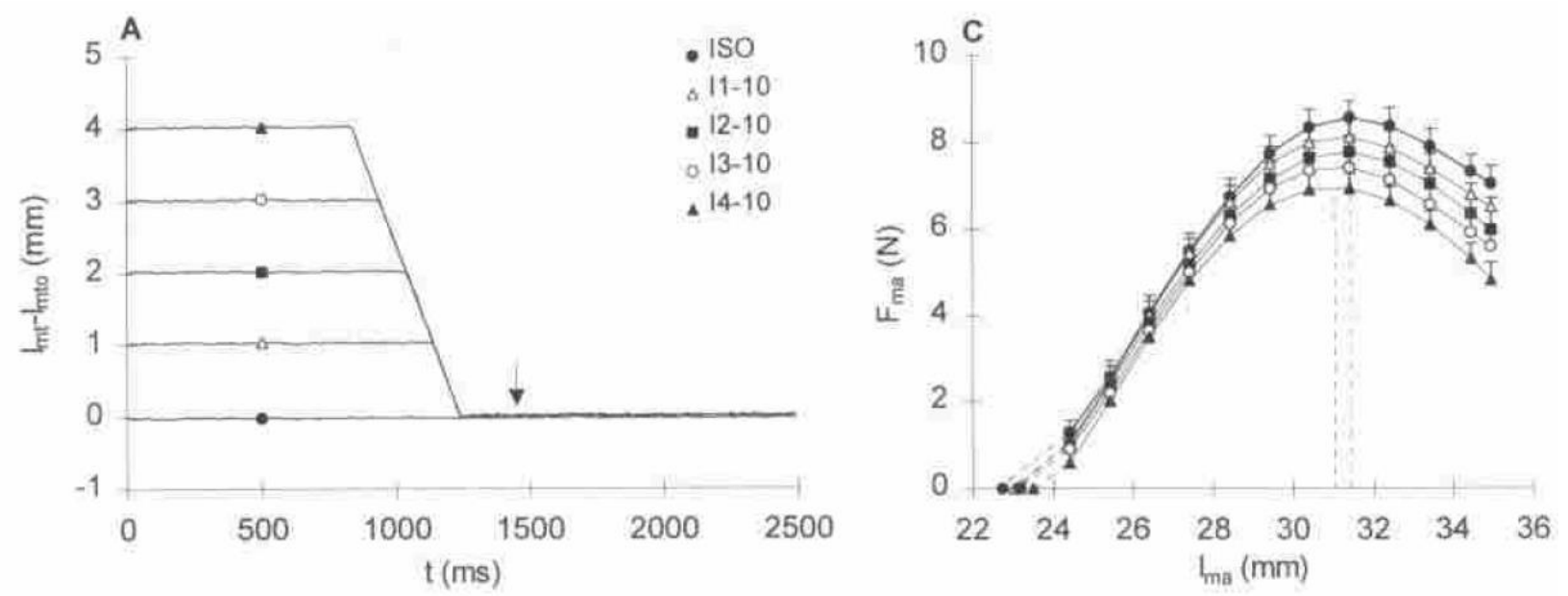

Figure 2.6 The effect of shortening-induced force depression on optimum MTU length.

Taken with permission from Meijer et al (1997). Force-length profiles of rat medial gastrocnemius obtained during isometric contractions (top curve) and varying degrees of shortening distance. The optimum length of the most force-depressed contractions was slightly shifted to shorter muscle lengths, in line with Holt and Williams' assertion. Importantly, however, these force-length profiles were of the entire MTU length, not the muscle fascicle length, which may affect the results (and explain why the measured length is so long).

\subsubsection{Does Increased Compliance Cause Shortening-Induced Force Depression?}

While the first issue at hand is whether shortening-induced force depression might impact the optimum length, a second issue is whether or not increases in in-series compliance even causes shortening-induced force depression as it is described in the literature. Typically, shortening-induced force depression is measured by stimulating a muscle isometrically, and then shortening the total MTU length to a shorter length while still activated. Conceivably, increased muscle shortening via in-series compliance elongation, while keeping MTU length constant, would still impart the same strain-dependent effects on the actin filaments, reducing the probability of cross-bridge formation and thus reducing the force potential at a given length. There is limited research in this area, however Mayfield, Launikonis, Cresswell, \& Lichtwark, (2016) reported substantially reduced force output and reduced force summation in Cane toad peroneus fibre bundles in vitro with an added compliant artificial tendon. In this setup, however, 
force outputs were reflected relative to the initial fibre length, and so much of the force reduction at a given initial length can be attributed to the fibres shortening onto the ascending limb. Mayfield et al attributed the losses in force and force summation primarily to reduced forcegenerating potential via shortening to the ascending limb, as well as via an increased shortening velocity, although they did mention the possibility that "history-dependent contractile properties, specifically those concerned with actin-myosin interaction subsequent to active shortening" may have also contributed. Whether the force depression is from one of these mechanisms or a combination of all three, it does seem reasonable that active shortening against the series elastic component (SEC) compliance would have the potential to decrease force by the same mechanism that it does in the more controlled experiments, and therefore is a key question that needs to be explored.

\subsection{The Potential Role of the Method of Calculation of Active Force on Activation-Dependent Shifts in Optimum Length}

Muscle force depends both on passive and active force. Passive force is exerted by tissues in the MTU, both in parallel with the muscle fibres (the so-called parallel elastic component PEC), and in series with the muscle fibres (the SEC). Passive force increases as muscle is stretched to longer muscle lengths, analogous to stretching a rubber band. Active force is exerted by the muscle cells by way of cross-bridge cycling that relies on energy from the hydrolysis of ATP to produce force, and, as we have been discussing, is dependent upon the muscle's length, as well as its history of contraction (among other things). In Section 2.1, we discussed the difference between truly isometric contractions and fixed-end contractions. Briefly, in a purely isometric contraction, the muscle fibre length does not change throughout the contraction, and therefore the length of the sarcomeres should be the same at the peak of 
contraction as they were prior to the contraction. However, in many experiments (including those performed here), the muscle is attached to the force transducer via its tendon, which, depending on the muscle used, can be compliant. In these fixed-end contractions, when the muscle contracts, the tendon stretches, and therefore, the muscle/sarcomere length decreases. These fixed-end contractions are thus not actually isometric in nature, and so the muscle/sarcomere length at the peak of contraction is shorter than its length prior to the contraction. Because the muscle/sarcomeres shorten, the passive force from the PEC would decrease during the contraction. Active force is calculated by subtracting the passive force from the total force during a contraction, and since the passive force decreases when the muscle shortens, it is inappropriate to subtract the passive force exerted prior to the contraction during fixed-end contractions, as is often done. In keeping with the terminology of MacIntosh (2017), this will be considered the "traditional method" of calculating active force. MacIntosh \& MacNaughton (2005) showed that this traditional method of calculating active force has the potential to underestimate the optimum length (see Figure 2.7 below). The more appropriate calculation is to subtract the passive force that is estimated to persist at the fascicle length at which peak force occurred during the contraction (MacIntosh \& MacNaughton, 2005). As above, this will be considered the "alternative method" of calculating active force. Regarding the potential for the method of calculation to affect any apparent activation-dependent shifts in optimum length, potentially, the underestimation resulting from the traditional method may be relative to the amount of force produced (associated with a greater magnitude of fascicle shortening). Therefore, the underestimation of optimum length may be relatively greater with maximal, high force contractions, than submaximal or twitch contractions. Additional compliance may exaggerate this problem (since shortening magnitude would increase further), as there would be a greater 
excursion on the passive force curve, magnifying the error in calculation. If this is the case, any activation dependent shifts seen may also depend on the method of calculation of active force used, with the traditional method of calculation potentially overestimating any shifts in optimum length.

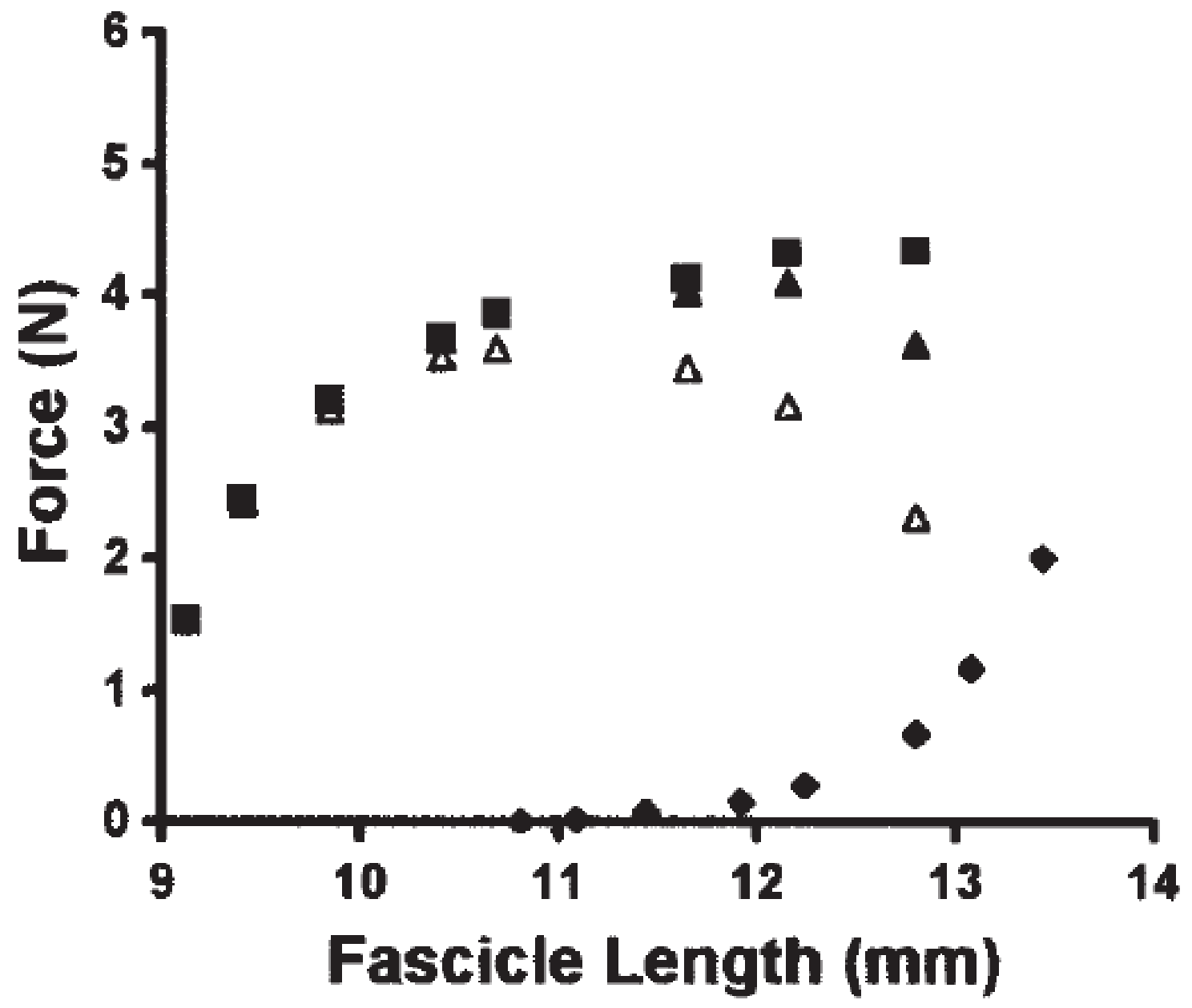

Figure 2.7 The effect of the method of active force calculation on the apparent optimum length.

Taken with permission from MacIntosh \& MacNaughton (2005), where fascicle length was measured with sonomicrometry. Total force (squares), passive force (diamonds), active force calculated the "traditional" way (open triangles), and active force calculated the "alternative way" (closed triangles). It is evident that accounting for decreases in passive force during a contraction can impact the apparent optimum length, whereby the traditional method will tend to underestimate the true optimum length by subtracting more passive force than appropriate at longer lengths. If this error is not consistent between different levels of activation, then perhaps this has the potential to influence the relative shift in optimum length that is seen at submaximal activation levels. 


\section{Addressing the Problem: Can Shortening-Induced Force Depression Explain Activation-Dependent Shifts in Optimum Length?}

\subsection{Introduction and Purpose}

The results of Holt and Azizi (2014) and Holt and Williams (2018) are quite curious, one reason being the large shifts in optimum length that they reported that don't seem to be compatible with our knowledge of isometric force-length relationships (see Figure 1.4). Notably, Holt and Williams did not study the relative shift in high- or low-compliant conditions in the same muscle preparation - they used two separate experimental setups to study the relative shift in each condition. Therefore, the assertion that the optimum length shifted leftward in the compliant condition was only inferred, but not directly compared. Based on the work of Meijer et al (1996) and Meijer et al (1997), this leftward shift seemed plausible, and if the degree of leftward shift was dependent on the activation, perhaps this would result in a relatively larger difference in optimum length when comparing the high and low activation conditions, explaining Holt and Williams' results. A hypothetical illustration of this notion is shown in Figure 3.1 below. 


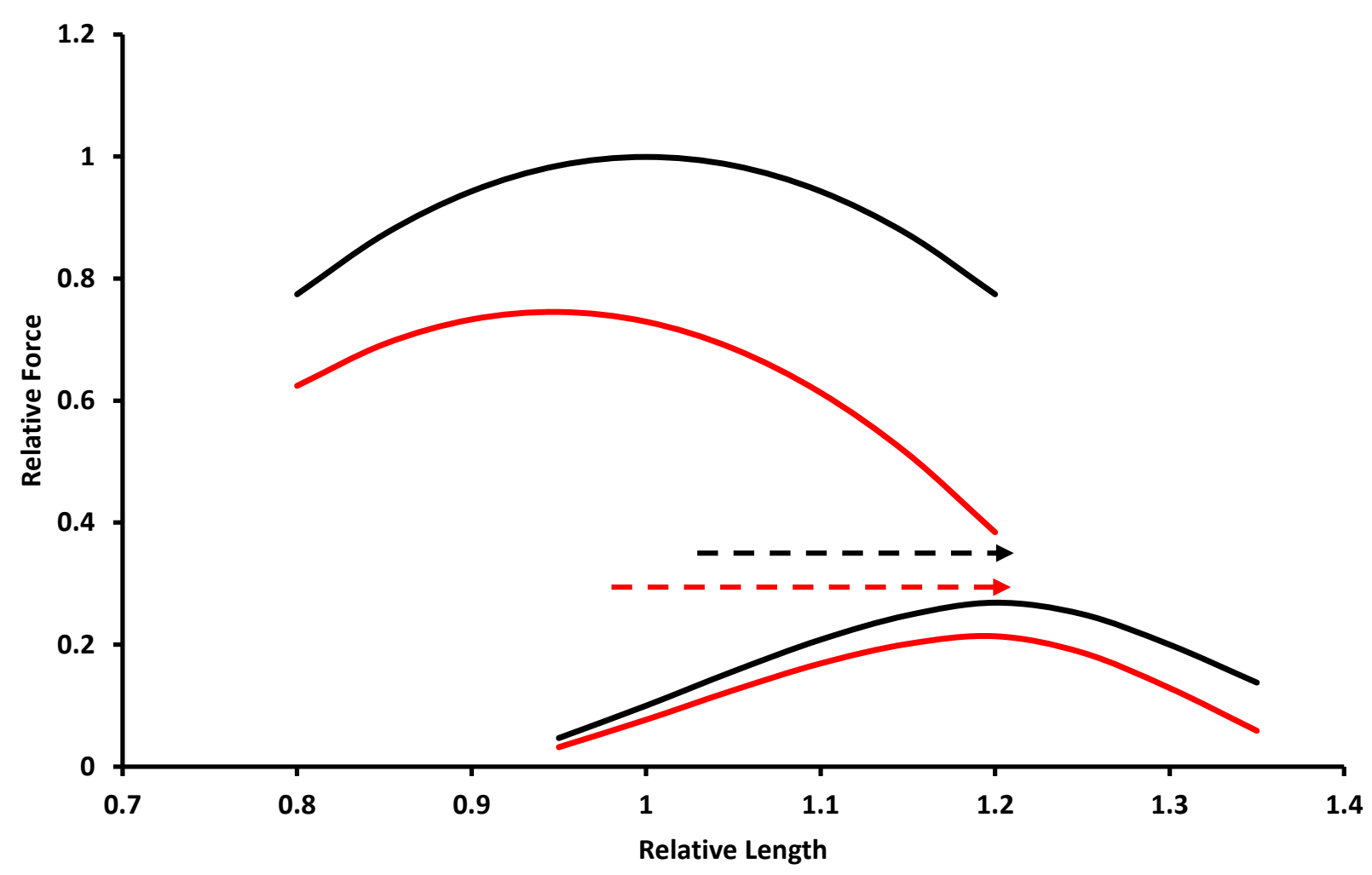

Figure 3.1 Theoretical illustration demonstrating how a length dependent increase in force depression may serve to increase the relative shift in optimum length.

Theoretical isometric high activation and low activation F-L curves are shown in black, with the activation dependent shift shown as the dotted black arrow. Theoretical force-depressed F-L curves are shown in red, with the larger activation-dependent shift shown as the red dotted arrow. Based on the findings of Meijer et al (1996) showing a length dependent increase in force depression and Meijer et al (1997) showing a decrease in optimum length with higher degrees of force depression. Taken together, this may have the potential to increase the activationdependent shift in optimum length, as the lower activation conditions would be less affected than higher activation conditions, leading to a larger relative shift in optimum length.

\subsection{Hypotheses and Formulation of Experimental Design}

As illustrated in Figure 3.1 above, the presence of shortening-induced force depression seems, on the surface, a plausible mechanism for a relatively greater activation-dependent shift in optimum length due to the length dependency of force depression. We wanted to look at this more closely, and so we needed to develop an experimental setup where we could determine the 
optimum length in high- and low-activation conditions, as well as high- and low-compliance conditions in the same muscle. We wanted to determine, firstly, if increased compliance led to a greater shift in optimum length with decreasing activation level, and secondly, if this was due to a rightward shift of the low-activation optimum length, or a leftward shift of the high-activation optimum length.

It was hypothesized that increased in-series compliance would lead to a reduction in force due to greater degrees of shortening-induced force depression. Additionally, it was hypothesized that the relative degree of force depression observed would be dependent on the level of activation, whereby higher activation conditions would be depressed to a larger extent than lower activation conditions due to the presumably increased distances shortened and higher forces produced. Due to this activation-dependence of force depression, it was hypothesized that the condition with higher series compliance might have a larger relative shift in optimum length compared to that of the condition with the lower series compliance.

It should be noted here that, while the mechanism described above seemed conceivable, the magnitude of difference seen between high and low compliance conditions by Holt and Williams (2018) seemed peculiarly large. Meijer et al (1998) reported a shift of only $\sim 1 \%$ of the MTU optimum length with the most force depressed contractions. Holt and Williams' reported a difference in relative shift (twitch optimum length divided by tetanic optimum length) of $\sim 30 \%$ of the tetanic optimum length between high and low compliance conditions. Thus, there was some skepticism regarding the difference we were expecting to see and because of this, we entertained some other ideas we thought may have the potential to influence the magnitude of this activation dependent shift observed. One of these factors was the method of calculation of active force, as detailed in Section 2.5. It was hypothesized that when calculating the active force 
the traditional way, any activation dependent shifts in optimum length would be magnified, due to an assumed greater relative error for contractions with greater shortening magnitudes.

One other such factor we surmised may have an effect on the shift observed was the presence of potentiation for the submaximal activation contractions. Activity-dependent potentiation refers to an enhanced contractile response (i.e. increased force output) as a result of prior contractile activity (MacIntosh \& Rassier, 2002). This potentiation is caused by phosphorylation of the myosin regulatory light chain (RLC), which, like the effects of decreased temperature discussed earlier on, has the effect of moving the myosin heads further away from the filament backbone, and closer to the actin molecule (Grange, Vandenboom, \& Houston, 1993). As potentiation increases $\mathrm{Ca}^{2+}$ sensitivity on its own by increasing the proximity of the myosin filament to actin, we hypothesized that perhaps the increase in $\mathrm{Ca}^{2+}$ sensitivity at longer lengths would be superfluous, and the relative shift in optimum length that occurs at submaximal $\left[\mathrm{Ca}^{2+}\right]$ would be less apparent, or even not apparent at all.

In order to explore these notions, our initial plan was to observe the relative shift in optimum length at high $\left[\mathrm{Ca}^{2+}\right]$, an "intermediate" $\left[\mathrm{Ca}^{2+}\right]$, a low $\left[\mathrm{Ca}^{2+}\right]$, and a high $\left[\mathrm{Ca}^{2+}\right]$ but with reduced motor unit recruitment, both with and without additional in-series compliance. We were interested in "teasing out" the effects of both $\left[\mathrm{Ca}^{2+}\right]$-dependent and $\left[\mathrm{Ca}^{2+}\right]$-independent effects on shifts in optimum length.

\subsection{Trials and Tribulations}

\subsubsection{General Overview}

This problem to be solved is a simple one on the surface, but proved to include several frustrating issues that forced us to vary the experimental protocols that we used. While the in situ rat medial gastrocnemius setup had been extensively used in our lab and would have been a 
convenient first option, we initially had wanted to use a less pennate muscle such that any potential confounding effects of changes in pennation angle during shortening were minimized. Attempts were made at using either the rat soleus, or the extensor digitorum longus (EDL). Unfortunately, both of these muscle preparations proved to be too delicate for the insertion of the sonomicrometry crystals that were to be used, and we had to go back to the medial gastrocnemius. Some additional dilemmas that we faced included: the issue of finding the "sweet spot" for the additional in-series compliance, the issue of force losses due to fatigue or other factors, and the issue of maintaining a quality connection between the nerve and the electrode cuff. These will all be addressed initially to give the reader a sense of the quandaries that were faced, and then touched upon in more detail as the methods of the preliminary experiments are presented.

\subsubsection{The "Sweet Spot" of Additional Compliance}

In our experimental setup, the in-series compliance was manipulated by attaching a short piece of silicon tubing in series with the tendon and force transducer. This tubing comes in a wide range of inherent compliances, and the compliance of a given piece can be increased or decreased by varying the length of it. The issue here is that, in order to compare optimum lengths between conditions, an entire force-length curve must be obtained, with data points past the plateau and at least slightly on the descending limb. If the additional compliance allows the muscle to shorten too much, at the longer starting lengths it may still shorten past the plateau and onto the ascending limb, such that a true optimum length could not be estimated. As the SEC compliance of the individual rats will naturally vary, finding a piece of silicon tubing that consistently allowed complete force-length curves to be obtained, but was still significantly more compliant than the silk cord used in the low-compliance condition, took some trial and error. 
Figure 3.2 shows the force-length tracings and average excursion paths (drawn as a straight line) from resting to maximal activation for a low-compliance condition and a condition in which the added in-series compliance was far too high. In this case, the added compliance allowed the muscle to shorten all the way to the ascending limb (as described by Mayfield et al (2016) earlier), which prevented us from identifying a plateau.

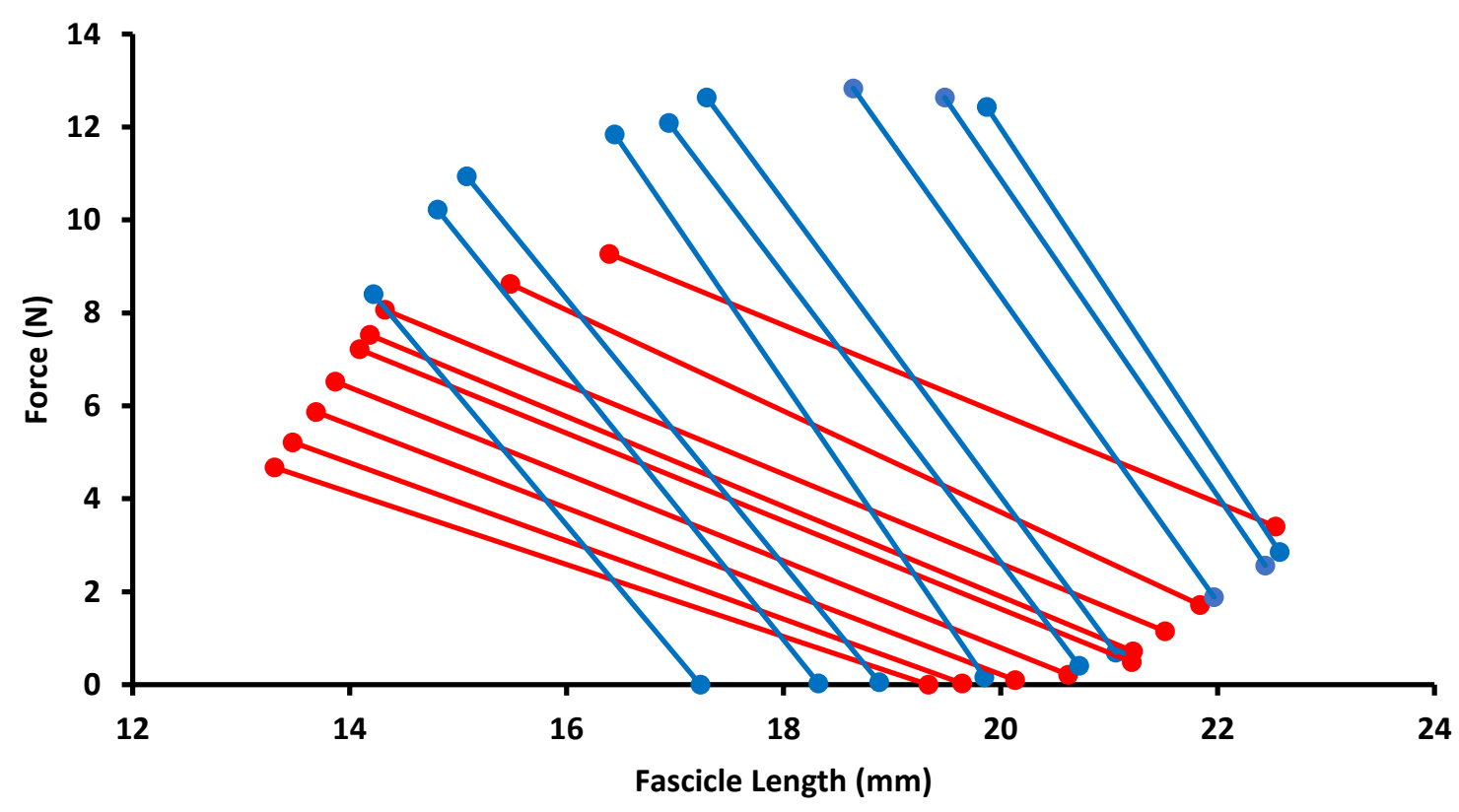

\section{Figure 3.2 The effect of extreme series compliance on the magnitude of muscle shortening and resultant force-length curve.}

Example force-length tracings (passive force curves and total force curves) of $100 \mathrm{~Hz}$ contractions in a low-compliant condition (blue) and a high-compliant condition (red), in which the additional in-series compliance was too large to allow a complete force-length curve to be obtained. Individual lines show the change in force over that change in length. Note: the starting length excursions shown are slightly different between conditions due to the inconsistencies in step length changes from the motor at this time. Also, during this time we were attempting to use larger rats for experiments in order to experiment with soleus or EDL, which is likely why the force outputs and fascicle lengths are larger than those presented later on. 


\subsubsection{Fatigue and/or Unexplained Losses in Force}

Muscular fatigue refers to a contractile response that is less than anticipated for a given stimulation (MacIntosh \& Rassier, 2002). The end-result of fatigue is a loss of force, but there are many potential "upstream" reasons for this loss in force. Impairment in any step in the process leading to muscular force production (detailed in Chapter 1) has the capacity to decrease the resultant force output. Reductions in motor neuron firing rate (Gandevia, 2001), accumulation of biproducts such as $\mathrm{H}^{+}$or inorganic phosphate (Sundberg \& Fitts, 2019), and failure at the neuromuscular junction (Sieck \& Prakash, 1995) have all been implicated in muscular fatigue, potentially leading to decreased $\mathrm{Ca}^{2+}$ release, decreased $\mathrm{Ca}^{2+}$ sensitivity, or a reduced force per cross-bridge (MacIntosh \& Rassier, 2002). In regard to experimental conditions that may lead to fatigue, repeated contractions, incomplete recovery periods, the frequency of stimulation or altered blood flow all may play a role. According to Sieck and Prakash (1995), "susceptibility to [neuromuscular transmission failure] increases with stimulation frequency and is most prevalent in fatigable fast-twitch motor units." Rat medial gastrocnemius is composed predominantly of fast-twitch fibres (Cornachione, Benedini-Elias, Polizello, Carvalho, \& Mattiello-Sverzut, 2011), and thus would be more likely to suffer from this type of fatigue than a more slow-twitch muscle like the soleus. Another potential experimental factor that may lead to fatigue is ischemia, which may reduce oxygenation of the muscle, or reduce biproduct removal, both of which may accelerate fatigue (Hepple, 2002; Murthy, Hargens, Lehman, \& Rempel, 2001). Altered blood flow or ischemia may result from compression of the blood vessels leading to the muscle; if the rat was positioned in a way that overstretched the thigh or calf muscles and occluded blood flow to the muscle being tested, this may result in undue fatigue despite an otherwise "non-fatiguing" stimulation protocol. Finally, while not technically classified as "fatigue," axonal degeneration from damage to the 
motor nerve after surgery may mimic muscular fatigue by a progressive loss of force due to additional motor neuron damage. According to Wang, Medress, \& Barres (2012), "as soon as a few minutes after axotomy, the axonal segments immediately proximal and distal to the injury site rapidly degenerate by several hundred micrometers in either direction in a process that lasts between 5 and 60 min (Kerschensteiner, Schwab, Lichtman, \& Misgeld, 2005; Wang et al., 2012). In our setup, the sciatic nerve was severed, and then the electrode nerve cuff was placed on the nerve stump. Due to this insult, and perhaps the ongoing irritation stemming from small shifts of the cuff on the nerve stump, axonal death may have progressed during the experiments, leading to an irreversible loss of force regardless of the stimulation protocol being used.

At this point, we need to consider how the above factors may affect the interpretation of our results. One of the key issues we were exploring was the depression of force after active shortening. Therefore, we needed to accurately account for any losses of force between low- and high-compliance conditions. Initially we took the approach of looking at the relative shift in optimum length between $100 \mathrm{~Hz}$ tetanic contractions, $50 \mathrm{~Hz}$ tetanic contractions, and twitch contractions in both high- and low-compliance conditions. In an attempt to mitigate any potential effect of fatigue over the course of the experiment, we alternated the order of conditions, such that some experiments began with the low-compliance condition first, and some began with the high-compliance condition first. What we began to find, however, was that the relative loss in force was very dependent upon the order in which we performed the experiment. When the lowcompliance condition was completed first, there was a significant decrease in force with the high-compliance condition. Based on the increase in shortening magnitude allowed by the added compliance, I assumed the reasoning for this loss in force was due to shortening-induced force depression. However, when the order was reversed and the high-compliance condition was 
completed first, we saw substantially less decrease in force between series, and in some cases the high-compliance condition actually produced more force than the low-compliance condition.

Obviously, there were factors other than shortening induced force depression here that were affecting our results. We began to remove some of the tetanic contractions being performed in the protocol to hopefully cause less fatigue over the duration of the experiment, which improved our consistency to some degree, but the effect was still apparent. Our final protocol involved very brief triple pulse stimuli at $5 \mathrm{~ms}$ pulse intervals rather than the longer duration tetanic contractions. This modification resulted in much more consistent results. It should be noted that without knowing the true reason for the losses in force we were seeing, improved surgical skill leading to less nerve damage may well have been what precipitated the improved results rather than any effect of varying the stimulation protocol. Regardless of whether the losses in force we observed were a result of true muscular fatigue due to any of the above mentioned mechanisms, or if it was due to progressive axonal death due to the sciatic nerve damage from the surgery and nerve cuff, the issue was resolved, and in our final protocol progressive losses in force were not apparent.

\subsubsection{Nerve Cuff Connection Quality}

In our experimental setup, the sciatic nerve was stimulated by a nerve cuff electrode that wrapped around the nerve stump. Maximal recruitment of all motor units was ensured by doubling the minimum voltage that maximized twitch active force. However, one of the issues that we were interested in exploring was the potential for a "calcium independent" shift in optimum length, as suggested by Holt and Azizi (2014). To do this, rather than varying stimulation frequency, which would impact calcium release, we would need to vary stimulation voltage as Holt and Azizi (2014) did, which would, theoretically at least, maintain a given $\left[\mathrm{Ca}^{2+}\right]$ 
in the active fibres, but would decrease the number of active motor units. We attempted to look at force-length curves of maximally activated $100 \mathrm{~Hz}$ contractions, and "submaximally" activated $100 \mathrm{~Hz}$ contractions, by lowering the stimulation voltage. One of the issues we faced was that, in our setup, the nerve stump/cuff is very close to the contracting muscle, and so any movement from the muscle, whether by stimulation, or by just passive length changes, had the potential to very slightly shift the position of the nerve cuff on the nerve stump. With less than maximal current, this motion could change the connectivity and may alter the number and/or location of the stimulated motor units, thereby changing the force output on any given contraction. Thus, a consistent force-length relationship could not be obtained because different levels of recruitment were happening at each of the lengths (see Figure 3.3 below). We were able to solve this issue by obtaining a smaller nerve cuff electrode that didn't get jostled by the muscle, and that fit more snugly along the nerve stump. Once we switched to this cuff, we were able to get more reliable force-length curves at a reduced voltage, in order to see if this affected the optimum length. 


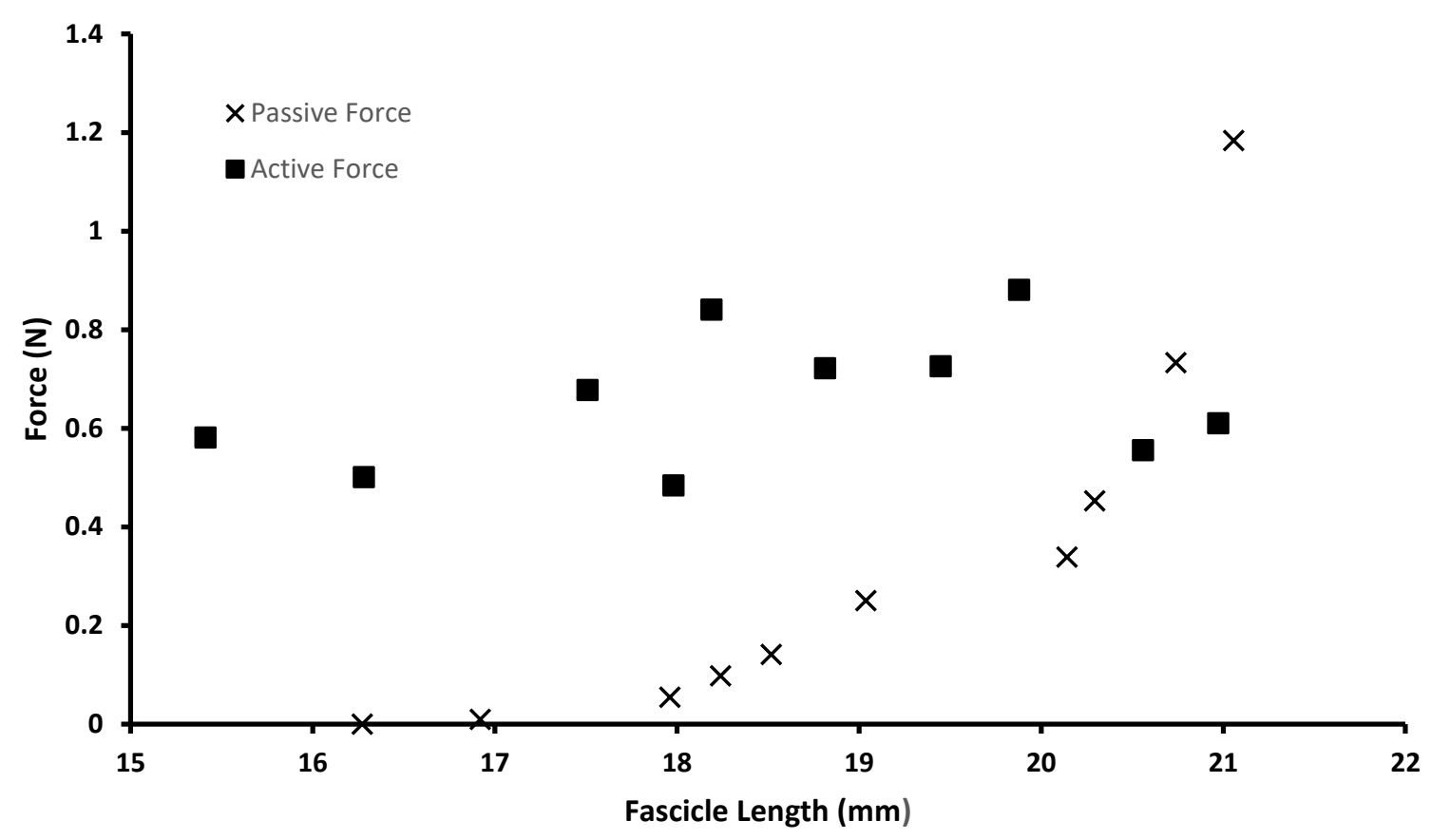

Figure 3.3 Example force-length relationship of the reduced voltage $100 \mathrm{~Hz}$ contractions.

Note the scattered active force data, likely due to the nerve cuff positioning being altered with changes in length before or during contractions, effectively changing the number of active motor units. I decided that pursuing this particular experimental test was not worthwhile until we could resolve this issue. Note that for some of these earlier experiments, a contraction at reference length $+5 \mathrm{~mm}$ was added to try to compensate for the shortening past the plateau that was occurring, which is why there are 10 data points on the figure.

\subsubsection{Sonomicrometry Signal Issues}

While the experimental issues noted above were certainly a nuisance, the most common issue that actually prevented me from including the entirety of data in my reports was poor quality sonomicrometry crystal traces that did not allow me to reliably obtain force-length traces for any of the stimulation conditions. Sonomicrometry transducers work by sending out and receiving sound energy to other nearby crystals. Based on the known speed of sound in the tissue the crystals are placed in (muscle, in this case), the distance between the crystals, and any changes in distance, can be calculated. This sonometric data, however, had the tendency to sometimes become incredibly noisy, to the point where no measurements of fascicle length could 
be discerned (see Figure 3.4 below). Problematically, due to the experimental setup, once the crystals were inserted and glued into the muscle and the animal was fixed in the frame, it became next to impossible to physically adjust these crystals in hopes of obtaining a better signal. In a few circumstances the noise seemed to spontaneously improve, but more often I was left with beautiful force traces, but with no way of reliably estimating fascicle length at any point, effectively nullifying the experiment.
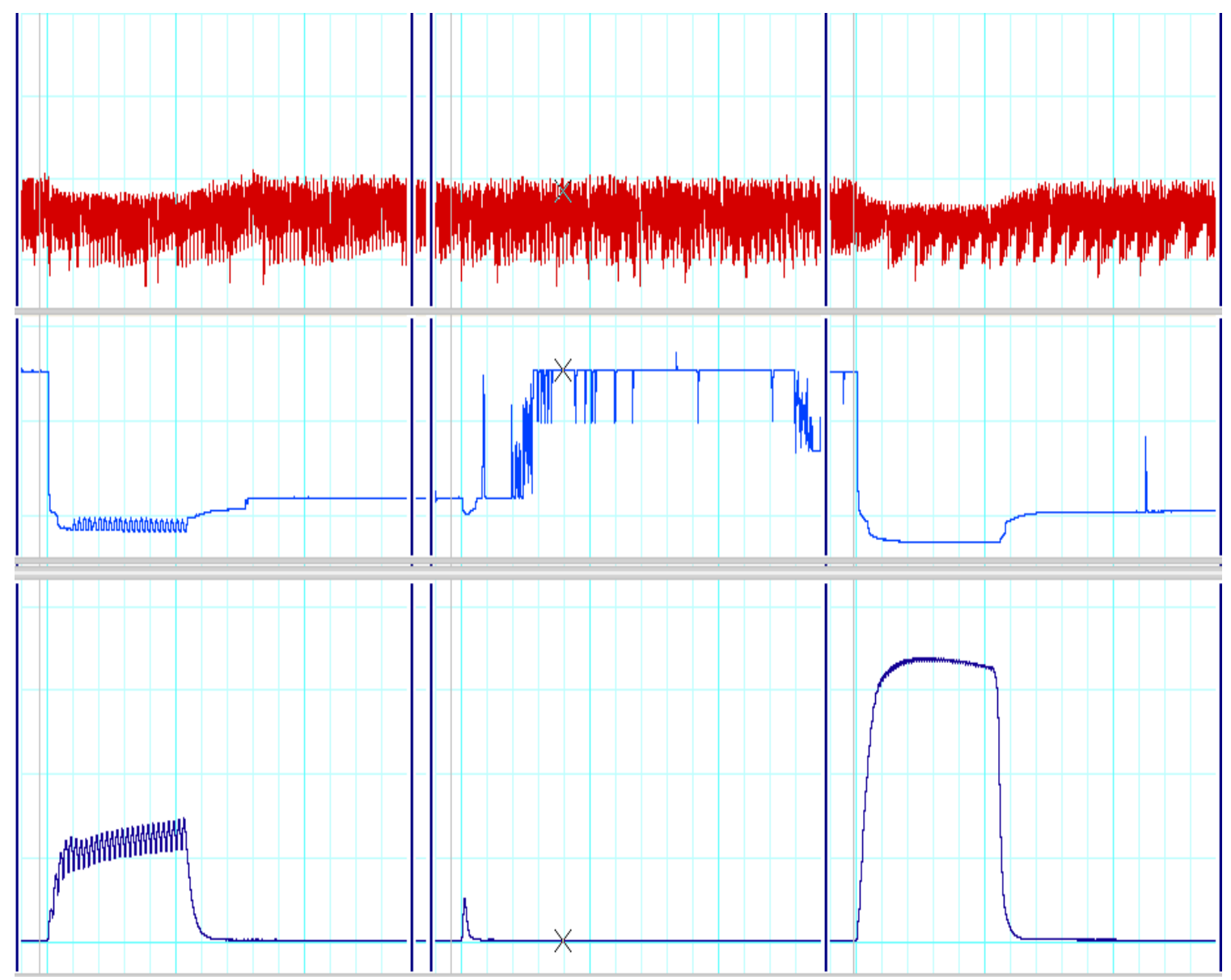

Figure 3.4 Raw data tracings of force and fascicle length.

The lower channel shows the force traces of a $50 \mathrm{~Hz}$ contraction, a twitch contraction, and a 100 $\mathrm{Hz}$ contraction. On the uppermost channel, the sonomicrometry data is too noisy for any accurate length measurements to be obtained. On the middle channel, the sonomicrometry data is showing stepwise length changes that may occur when one of the crystals does not receive the proper signal from the other. In both cases, fascicle length data would not be valid, preventing the proper force-length curves to be obtained. 


\subsection{Experimental Inclusion Criteria}

With all of the above said, it should be noted what the inclusion criteria were for including the experimental data in this and the following chapters. The most common reason for not including the results of a particular experiment in the analysis was due to the sonomicrometry data not allowing any reasonable fascicle length measurements to be derived. Additionally, on several occasions the force-length curves proved to be incomplete due to "overshortening" onto the ascending limb, precluding the calculation of peak force or optimum length since no plateau was observed. Finally, on some rare occasions, the animal expired before all of the stimulation protocols were finished, thus preventing the comparison of the data. With these points in mind, experiments were included in the analysis if they met three basic criteria: firstly, all stimulation protocols were complete before the animal expired; secondly, if the sonomicrometry traces were of sufficient quality to obtain valid length measurements, and finally, if the force-length traces obtained included a plateau region in which peak force and optimum length could be estimated. Overall, there were 23 experiments that met most or all criteria (see below for exception) and were subsequently analyzed (15 preliminary experiments and 8 final experiments). There were several experiments in which the quality of the sonometric data was too poor to utilize and thus were not analyzed, and several experiments where the animal expired before the completion of all protocols. The only exception to these criteria occurs in this chapter, in which several of the twitch force-length curves did not show a plateau, but for the sake of illustration, these experiments were kept in the analysis. In these few cases, the peak force and optimum length was estimated from the highest force output measured, and the corresponding length at which it occurred. This was not an issue in our final experimental approach detailed in Chapter 4. 


\subsection{Preliminary Experimental Methods, Results and Discussion}

\subsubsection{General Outline}

Rather than a more formal structure, the information presented here will follow a chronological organization which will encompass the methods, results and discussion, as well as rationale for refinement. This should allow a clearer elucidation of the thought processes that led to the final approach.

\subsubsection{Experimental Protocol Overview}

The experimental preparation will be explained in more detail further on. In short, the anesthetized rat's left hindlimb was shaved and its medial gastrocnemius muscle isolated and attached to a force transducer directly via a silk cord, or with an additional piece of silicon tubing attached in series with the muscle and tendon. Two sonomicrometry crystals were inserted along the length of a fascicle to measure fascicle length changes. An electrode cuff was placed around a stump of the sciatic nerve and stimulated via a Grass S88 Stimulator (Grass Instruments, Quincy, MA, USA). Maximal stimulation voltage was found by measuring the force output of twitch contractions, while decreasing the stimulation voltage from $\sim 3 \mathrm{~V}$ downwards to the lowest voltage that still resulted in maximal twitch force. This voltage was then doubled for the remainder of the experiment (other than where noted), to ensure maximal motor unit recruitment during the contractions. The reference length was found by stimulating the muscle with two pulses at $5 \mathrm{~ms}$ interval at a range of lengths, until the length at which the highest active force (total force minus passive force; traditional active force) was obtained. From here, force-length curves were obtained by stimulating at this reference length and varied at $1 \mathrm{~mm}$ intervals $( \pm$ 4mm). The specific stimulation protocol used evolved over the course of the data collection process, as we attempted to refine the protocol in order to dial in on the most important aspects 
of the experiment, and to minimize any confounding factors. Each specific protocol we used in these preliminary experiments will be briefly explained, and the rationale for varying the protocol will be given and expanded upon within this chapter.

\subsubsection{Data Analysis Overview}

For each experiment, force and length data were measured in LabChart (ADI Instruments, Colorado Springs, CO, USA) at a sampling rate of $2000 \mathrm{~Hz}$, and then exported to Microsoft Excel (Microsoft Corporation, Redmond, WA, USA) and SPSS (IBM Corporation, Armonk, NY, USA) for analysis. The low-compliance $100 \mathrm{~Hz}$ contractions were used as the "norm" for comparative purposes, so the estimated peak force and optimum length of the other conditions were expressed relative to those values of the low-compliance $100 \mathrm{~Hz}$ contractions for a given experiment. The estimated peak force and optimum length was found by a $3^{\text {rd }}$ order polynomial fit of the low-compliance $100 \mathrm{~Hz}$ contractions. The peak force and optimum length of the other conditions were calculated the same way, and then expressed relative to this lowcompliance $100 \mathrm{~Hz}$ condition. In the event that a plateau was not observed, the highest force value and the corresponding length were taken as a proxy for the optimum length. Two-way repeated measures ANOVA were done to determine any effect that additional in-series compliance had on the relative force output and optimum length of each contraction type.

\subsubsection{Stimulation Protocol Version 1}

At each length (reference length $\pm 4 \mathrm{~mm}$ ), the muscle was stimulated in the following order: 1 . Twitch, $2.50 \mathrm{~Hz}, 3.100 \mathrm{~Hz}$, with $1 / 2$ of the minimum voltage that maximized twitch active force, $4.100 \mathrm{~Hz}, 5$. Twitch. These contractions were approximately 5 seconds apart, and the muscle was given 5 minutes to recover before repeating the series of contractions at the next length. All tetanic contractions were $300 \mathrm{~ms}$ in duration (see Figure 3.5 below for an example of 
the force tracings of each of these contractions). Once the full length excursion had been tested, the in-series attachment was changed and then the entire series was repeated with the alternate compliance condition. There were a couple of considerations that led us to this particular protocol. Firstly, the motor we were using at the time was fairly inconsistent in its step length changes. I wanted to have consistent fascicle lengths to compare between the different conditions, and for this reason I opted to perform the various contraction types in rapid succession at a consistent length, rather than completing entirely separate force-length protocols for each condition. The specific order of the contractions was chosen in an "ascending" manner, in order to minimize any effects of potentiation or fatigue on the subsequent contraction, with the very last contraction after the $100 \mathrm{~Hz}$ contractions meant to be a potentiated twitch, so that we could determine any effect that potentiation may have on the shift in optimum length.

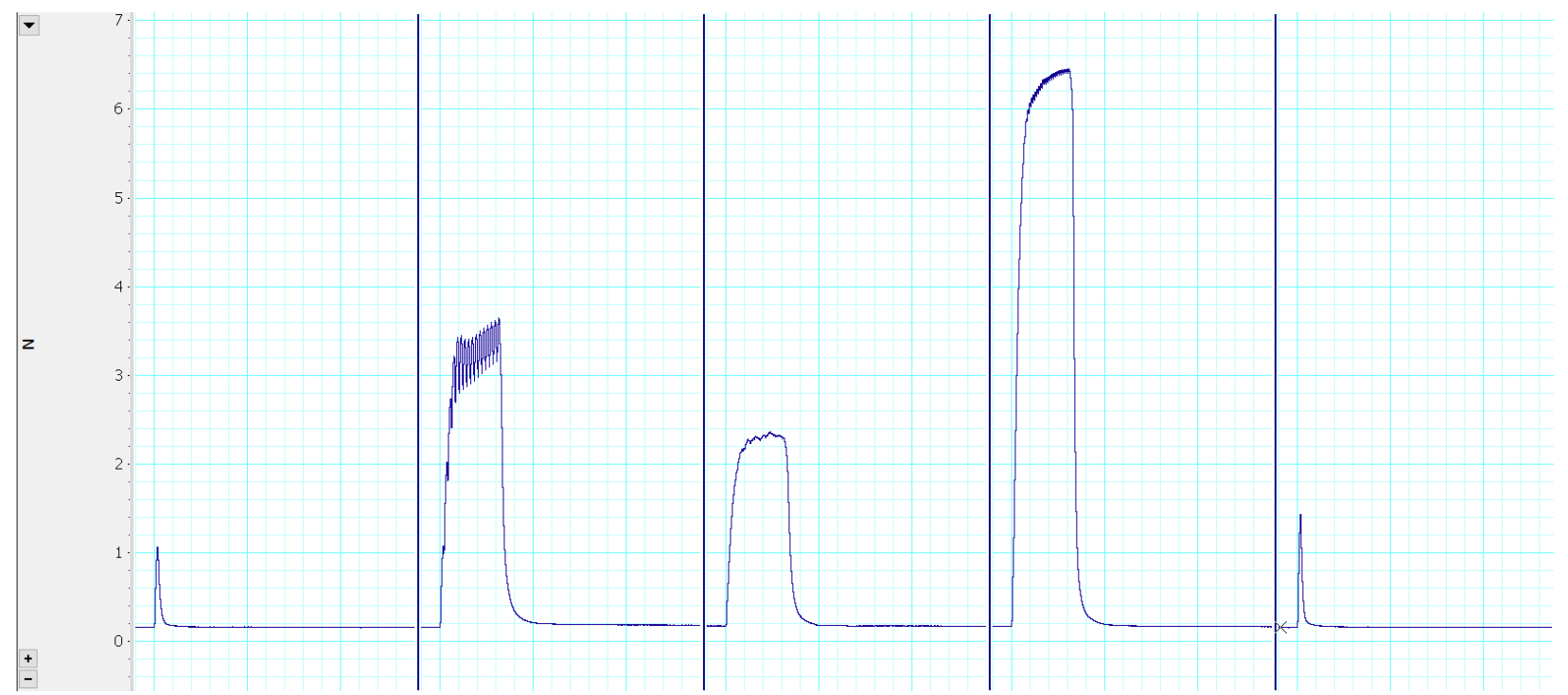

\section{Figure 3.5 Sample force tracings of twitch, $50 \mathrm{~Hz}, 100 \mathrm{~Hz}$ with reduced voltage, $100 \mathrm{~Hz}$, and potentiated twitch contractions.}

Raw data tracings showing the force tracing for each of the five contraction conditions that were tested. Contractions were recorded in succession at each length (reference length $\pm 4 \mathrm{~mm}$ ), approximately 5 seconds apart, with 5 minutes recovery between each series of contractions. Note the increased force output of the final twitch contraction due to the potentiation from the preceding contractions. 
Table 3.1 Relative peak force and relative optimum length of twitch, $50 \mathrm{~Hz}$, submaximal voltage $100 \mathrm{~Hz}, 100 \mathrm{~Hz}$, and potentiated twitch contractions with and without additional in-series compliance

\begin{tabular}{|c|c|c|c|c|c|c|}
\hline \multirow{2}{*}{ Condition } & \multicolumn{2}{|c|}{$\underline{\text { Relative Peak Force }}$} & \multicolumn{2}{|c|}{$\underline{\text { Relative Optimum Length }}$} & \multicolumn{2}{|c|}{$\begin{array}{l}\text { Compliance at Reference } \\
\text { Length }\left(\mathrm{mm} \cdot \mathrm{N}^{-1}\right)\end{array}$} \\
\hline & $\begin{array}{c}\text { Low } \\
\text { Compliance }\end{array}$ & $\begin{array}{c}\text { High } \\
\text { Compliance }\end{array}$ & $\begin{array}{c}\text { Low } \\
\text { Compliance }\end{array}$ & $\begin{array}{c}\text { High } \\
\text { Compliance }\end{array}$ & $\begin{array}{c}\text { Low } \\
\text { Compliance }\end{array}$ & $\begin{array}{c}\text { High } \\
\text { Compliance }\end{array}$ \\
\hline Twitch & $0.21 \pm 0.02$ & $0.19 \pm 0.02$ & $1.27 \pm 0.03$ & $1.30 \pm 0.04$ & $0.53 \pm 0.05$ & $0.87 \pm 0.08$ \\
\hline $\begin{array}{l}50 \mathrm{~Hz} \\
\text { Reduced Voltage }\end{array}$ & $0.71 \pm 0.07$ & $0.67 \pm 0.05$ & $1.21 \pm 0.04$ & $1.24 \pm 0.04$ & $0.38 \pm 0.04$ & $0.59 \pm 0.10$ \\
\hline $100 \mathrm{~Hz}$ & $0.62 \pm 0.11$ & $0.49 \pm 0.19$ & $1.09 \pm 0.04$ & $1.07 \pm 0.06$ & $0.48 \pm 0.05$ & $0.65 \pm 0.14$ \\
\hline $\begin{array}{l}100 \mathrm{~Hz} \\
\text { Potentiated }\end{array}$ & $1.00 \pm 0.00$ & $0.93 \pm 0.05$ & $1.00 \pm 0.00$ & $1.01 \pm 0.02$ & $0.53 \pm 0.09$ & $0.72 \pm 0.14$ \\
\hline Twitch & $0.29 \pm 0.02$ & $0.27 \pm 0.03$ & $1.17 \pm 0.02$ & $1.24 \pm 0.04$ & $0.54 \pm 0.04$ & $0.85 \pm 0.09$ \\
\hline
\end{tabular}

Peak force and optimum length expressed relative to the $100 \mathrm{~Hz}$ low-compliance condition. Values are mean $\pm \operatorname{SEM}(\mathrm{n}=4)$.

Separate two-way repeated measures ANOVA were done to determine the effect of activation condition and compliance on the relative force output and relative optimum length. There was no significant interaction between activation level and compliance condition on either relative force $(\mathrm{p}=0.328)$ or optimum length $(\mathrm{p}=0.238)$. The main effect of activation level expectedly showed a significant effect on both force output $(\mathrm{p}<0.001)$, as well as optimum length $(\mathrm{p}<0.001)$. On the other hand, there was no significant effect of additional in-series compliance on either force output ( $\mathrm{p}=0.157)$, or optimum length $(\mathrm{p}=0.324)$. Post hoc tests using a Bonferroni correction (combining compliance conditions) showed that there was a significant reduction in force output in comparison to the $100 \mathrm{~Hz}$ contractions for both the twitch and the potentiated twitch contractions, but due to the high variance and low sample size of the data, no significant difference was detected in either the $50 \mathrm{~Hz}$ or the reduced voltage $100 \mathrm{~Hz}$ contractions. There was, however, a significant increase in force output between the twitch contractions and the potentiated twitches $(\mathrm{p}=0.016)$. In looking at changes in optimum length, the twitch optimum length was significantly greater than the $100 \mathrm{~Hz}$ optimum length $(\mathrm{p}=0.031)$, however despite the 
seemingly large differences in the other values, none of the other activation conditions' optimum length was significantly different than the $100 \mathrm{~Hz}$ condition. See Table 3.1 for full results.

Due to the very small sample size, any statistical test results are not particularly meaningful. Having said that, from these four experiments it was clear from the quality of data I was getting that the reduced voltage contractions were not going to be useful to us (see Figure 3.2). Therefore, I made the decision to take those contractions out of the protocol for the time being, and continue on.

\subsubsection{Stimulation Protocol Version 2}

Due to the issue of the variability of the nerve cuff connection, I opted to remove the reduced voltage contractions from the experimental protocol. The rest of the protocol was identical to the above: at each length (reference length $\pm 4 \mathrm{~mm}$ ), the muscle was stimulated in the following order: 1 . Twitch, 2. $50 \mathrm{~Hz}, 3.100 \mathrm{~Hz}, 4$. Twitch. These contractions were approximately 5 seconds apart, and the muscle was given 5 minutes to recover before repeating the series of contractions at the next length. Once the full -length excursion had been tested, the in-series attachment was changed and then the entire series was repeated with the alternate compliance condition. 
Table 3.2 Relative peak force and relative optimum length of twitch, $50 \mathrm{~Hz}$, $100 \mathrm{~Hz}$, and potentiated twitch contractions with and without additional in-series compliance

\begin{tabular}{|c|c|c|c|c|c|c|}
\hline \multirow[t]{2}{*}{ Condition } & \multicolumn{2}{|c|}{$\underline{\text { Relative Peak Force }}$} & \multicolumn{2}{|c|}{$\underline{\text { Relative Optimum Length }}$} & \multicolumn{2}{|c|}{$\begin{array}{l}\text { Compliance at Reference } \\
\text { Length }\left(\mathrm{mm} \cdot \mathrm{N}^{-1}\right)\end{array}$} \\
\hline & $\begin{array}{c}\text { Low } \\
\text { Compliance }\end{array}$ & $\begin{array}{c}\text { High } \\
\text { Compliance }\end{array}$ & $\begin{array}{c}\text { Low } \\
\text { Compliance }\end{array}$ & $\begin{array}{c}\text { High } \\
\text { Compliance }\end{array}$ & $\begin{array}{c}\text { Low } \\
\text { Compliance }\end{array}$ & $\begin{array}{c}\text { High } \\
\text { Compliance }\end{array}$ \\
\hline Twitch & $0.22 \pm 0.03$ & $0.21 \pm 0.02$ & $1.26 \pm 0.05$ & $1.29 \pm 0.05$ & $0.52 \pm 0.09$ & $0.90 \pm 0.13$ \\
\hline $50 \mathrm{~Hz}$ & $0.69 \pm 0.03$ & $0.55 \pm 0.02$ & $1.19 \pm 0.05$ & $1.18 \pm 0.05$ & $0.36 \pm 0.05$ & $0.73 \pm 0.11$ \\
\hline $\begin{array}{l}100 \mathrm{~Hz} \\
\text { Potentiat }\end{array}$ & $1.00 \pm 0.00$ & $0.83 \pm 0.08$ & $1.00 \pm 0.00$ & $1.02 \pm 0.03$ & $0.42 \pm 0.07$ & $0.73 \pm 0.13$ \\
\hline Twitch & $0.27 \pm 0.02$ & $0.26 \pm 0.04$ & $1.21 \pm 0.05$ & $1.26 \pm 0.04$ & $0.58 \pm 0.10$ & $0.86 \pm 0.13$ \\
\hline
\end{tabular}

Peak force and optimum length expressed relative to the $100 \mathrm{~Hz}$ low-compliance condition. Values are mean \pm SEM $(n=6)$.

Separate two-way repeated measures ANOVA were done to determine the effect of activation condition and compliance on the relative force output and relative optimum length. There was a significant interaction effect between activation level and compliance on force output ( $\mathrm{p}=0.014)$, but not optimum length $(\mathrm{p}=0.093)$. There was a significant main effect of activation level on optimum length ( $\mathrm{p}<0.001)$, but no significant effect of compliance $(\mathrm{p}=0.200)$. Expectedly, the twitch and potentiated twitch optimum length was significantly greater than that of the $100 \mathrm{~Hz}$ contractions ( $\mathrm{p}=0.007, \mathrm{p}=0.021$, respectively), and while the mean optimum length of the $50 \mathrm{~Hz}$ contractions was greater than that of the $100 \mathrm{~Hz}$ contractions, this difference was not statistically significant $(\mathrm{p}=0.06)$. Since there was a significant interaction between activation level and compliance on force output, multiple one-way repeated measures ANOVA were performed to determine simple main effects. There was no significant difference in force output between the low- and high compliance twitch contractions $(\mathrm{p}=0.259)$, potentiated twitch contractions ( $\mathrm{p}=0.671)$, or $100 \mathrm{~Hz}$ contractions $(\mathrm{p}=0.094)$, but there were significant differences with the $50 \mathrm{~Hz}$ contractions ( $\mathrm{p}=0.02$ ). The lack of significant difference at $100 \mathrm{~Hz}$ was likely due to the fact that on one of the experiments in which the high-compliance protocol was performed 
first, there was a large reduction in force by the time the low-compliance $100 \mathrm{~Hz}$ contractions were performed, to the point where there was $17 \%$ greater force production in the high compliance condition. This increased variability would make it difficult to identify differences that might otherwise be significant. See Table 3.2 for full results of this protocol.

Evidently, I was still having some issues as far as variability in the data, where the order use in the protocol was affecting the results. Before continuing, I grouped data from both of the previous protocols together to improve the sample size and allow better comparisons of the data.

\subsubsection{Combined Data from Stimulation Protocol Versions 1 and 2}

While the experimental protocols were not identical, to increase the sample size, the twitch, $50 \mathrm{~Hz}, 100 \mathrm{~Hz}$ and potentiated twitch force and length data were compared, while omitting the data from the reduced voltage $100 \mathrm{~Hz}$ contractions.

Table 3.3 Relative peak force and relative optimum length of twitch, $50 \mathrm{~Hz}$, $100 \mathrm{~Hz}$, and potentiated twitch contractions with and without additional in-series compliance

\begin{tabular}{|c|c|c|c|c|c|c|}
\hline \multirow[t]{2}{*}{ Condition } & \multicolumn{2}{|c|}{$\underline{\text { Relative Peak Force }}$} & \multicolumn{2}{|c|}{ Relative Optimum Length } & \multicolumn{2}{|c|}{$\begin{array}{l}\text { Compliance at Reference } \\
\text { Length }\left(\mathrm{mm} \cdot \mathrm{N}^{-1}\right)\end{array}$} \\
\hline & $\begin{array}{c}\text { Low } \\
\text { Compliance }\end{array}$ & $\begin{array}{c}\text { High } \\
\text { Compliance }\end{array}$ & $\begin{array}{c}\text { Low } \\
\text { Compliance }\end{array}$ & $\begin{array}{c}\text { High } \\
\text { Compliance }\end{array}$ & $\begin{array}{c}\text { Low } \\
\text { Compliance }\end{array}$ & $\begin{array}{c}\text { High } \\
\text { Compliance }\end{array}$ \\
\hline Twitch & $0.22 \pm 0.02$ & $0.20 \pm 0.02$ & $1.27 \pm 0.03$ & $1.30 \pm 0.03$ & $0.52 \pm 0.05$ & $0.89 \pm 0.08$ \\
\hline $50 \mathrm{~Hz}$ & $0.70 \pm 0.03$ & $0.60 \pm 0.02$ & $1.20 \pm 0.03$ & $1.20 \pm 0.03$ & $0.37 \pm 0.03$ & $0.68 \pm 0.08$ \\
\hline $\begin{array}{l}100 \mathrm{~Hz} \\
\text { Potentiate }\end{array}$ & $1.00 \pm 0.00$ & $0.87 \pm 0.05$ & $1.00 \pm 0.00$ & $1.02 \pm 0.02$ & $0.46 \pm 0.06$ & $0.73 \pm 0.09$ \\
\hline Twitch & $0.28 \pm 0.01$ & $0.26 \pm 0.02$ & $1.20 \pm 0.03$ & $1.26 \pm 0.03$ & $0.56 \pm 0.06$ & $0.86 \pm 0.08$ \\
\hline
\end{tabular}

Peak force and optimum length expressed relative to the $100 \mathrm{~Hz}$ low-compliance condition.

Values are mean $\pm \operatorname{SEM}(n=10)$. 


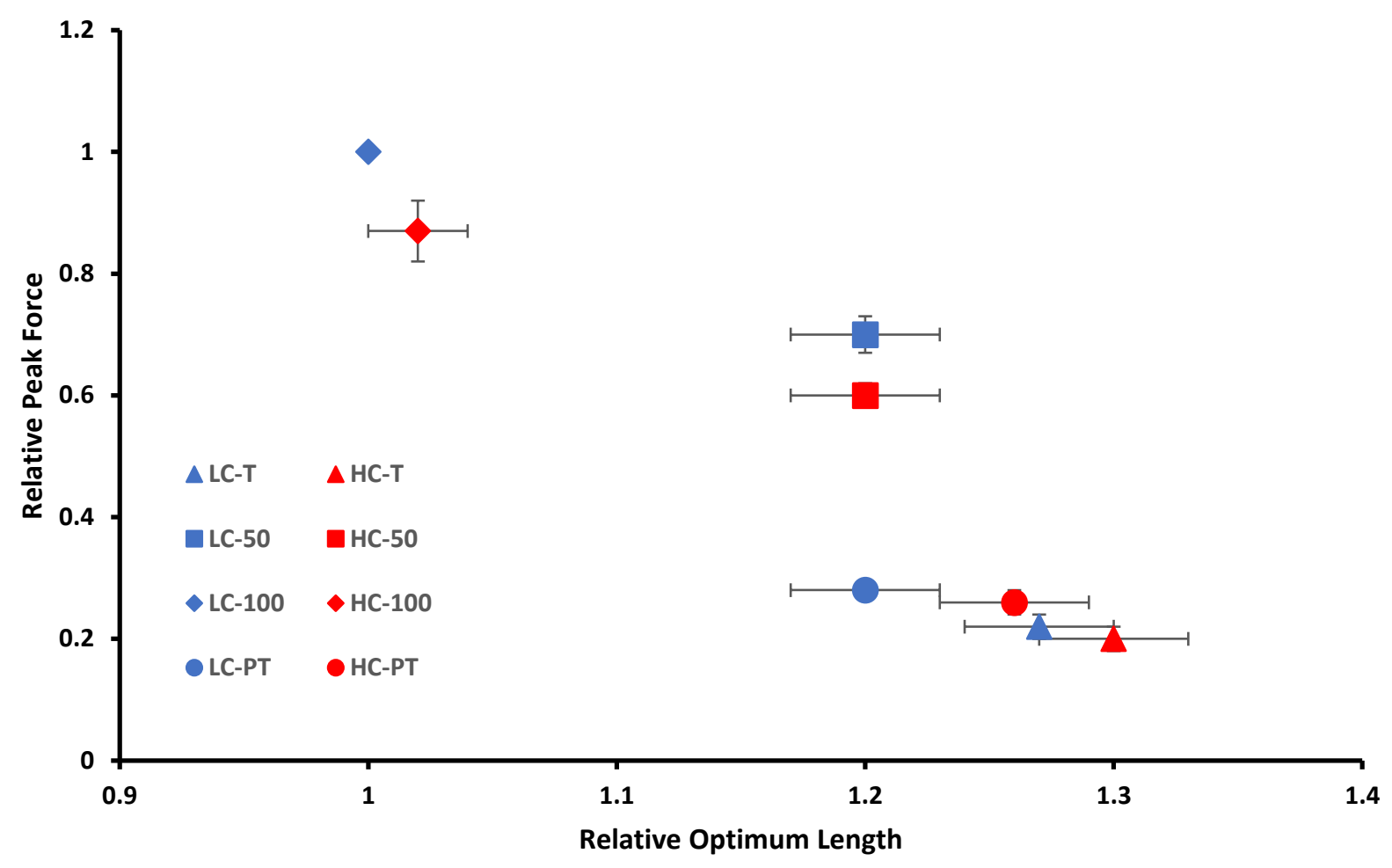

Figure 3.6 Preliminary experimental data showing relative peak force and relative optimum length for twitch, $50 \mathrm{~Hz}, 100 \mathrm{~Hz}$, and potentiated twitch in low- and high compliance conditions.

Data points are mean \pm SEM for $n=10$ experiments. Red $=$ high compliance $(H C)$, blue $=$ low compliance $(\mathrm{LC})$. Diamonds $=100 \mathrm{~Hz}$, squares $=50 \mathrm{~Hz}$, triangles $=$ twitches, circles $=$ potentiated twitches. There is a trend for reductions in force due to the additional in-series compliance, but no significant changes in optimum length at any activation condition apart from potentiated twitches.

Separate two-way repeated measures ANOVA were done to determine the effect of activation condition and compliance on the relative force output and relative optimum length.

When all data were combined, there was a significant interaction effect between activation level and compliance on both force output $(\mathrm{p}=0.008)$, and optimum length $(\mathrm{p}=0.01)$ Multiple one-way repeated measure ANOVA tests were done to determine simple main effects. In the low compliance condition, there were significant differences in optimum length between the $100 \mathrm{~Hz}$ contractions and all other activation levels (twitch, $50 \mathrm{~Hz}$, and potentiated twitch). Additionally, 
the potentiated twitch optimum length was significantly reduced compared to the non-potentiated twitch $(\mathrm{p}=0.004)$. In the high compliance condition, again there were significant differences in optimum length between the $100 \mathrm{~Hz}$ contractions and all other activation levels (twitch, $50 \mathrm{~Hz}$, and potentiated twitch), however, there was no significant difference in optimum length between potentiated and non-potentiated twitch contractions $(\mathrm{p}=0.071)$. Regarding the force output, there were significant differences between all activation conditions in both the low-compliance and high-compliance conditions. At a given level of activation, there were no significant differences in optimum length with twitch contractions, $50 \mathrm{~Hz}$, or $100 \mathrm{~Hz}$, but there was a significant difference in the potentiated twitch optimum length between the low- and high-compliance conditions. There were significant reductions in force output in the high-compliance condition compared to the low-compliance condition with twitch contractions ( $\mathrm{p}=0.032), 50 \mathrm{~Hz}$ contractions $(\mathrm{p}=0.011,100 \mathrm{~Hz}$ contractions $(\mathrm{p}=0.041)$, but not the potentiated twitch contractions $(p=0.315)$. See Table 3.3 and Figure 3.6 for full results of the combined data.

Overall, when grouped together we saw that generally, additional in-series compliance had little, if any effect on optimum length, but that it did cause reductions in force for all levels of activation except for the potentiated twitch.

Interestingly, the optimum length of the potentiated twitch was significantly less than that of the non-potentiated twitch in the low-compliant condition, showing that potentiation does have the potential to impact the magnitude of the shift in optimum length. Nevertheless, there was still a shift seen, which suggests that there was still a length-dependent increase in $\mathrm{Ca}^{2+}$ sensitivity, despite the increased proximity of the myosin heads and actin filaments due to myosin light chain (MLC) phosphorylation. This reduced shift in optimum length with potentiated twitches compared to non-potentiated twitches can be explained by the interaction 
and redundancy of the effects that RLC phosphorylation and changes in length have on $\mathrm{Ca}^{2+}$ sensitivity. As RLC phosphorylation would bring the myofilaments closer together to begin with, the muscle would not have to lengthen as much to get to a point where the length-dependent decrease in lattice spacing effectively "outweighed" the reduced filament overlap. Additionally, it has been shown that potentiation is length-dependent (Rassier \& Macintosh, 2002), whereby potentiation enhances force output at shorter lengths but not longer lengths, again due to the redundancy in effects of RLC phosphorylation and increases in length. This relative increase in force output at relatively shorter lengths may have the potential to shift the apparent optimum length to shorter lengths on its own, thereby decreasing the shift when compared to the $100 \mathrm{~Hz}$ contractions.

From these data, it may have seemed that we had a fairly good idea that the additional inseries compliance reduced force output but did not seem to cause a shift in optimum length. This would seem to go against the hypothesis set out by Holt and Williams (2018), where they supposed that additional in-series compliance would increase the amount of shortening-induced force depression, and thereby cause a decrease in optimum length. We did see a decrease in force with the increased shortening magnitude, but without a concomitant change in optimum length. The issue, however, was the discrepancy in results based on the order in which we performed the experimental protocols. I had tried to alternate the protocol order, whereby in half of the experiments the low-compliance protocol was done first, and in half of the experiments the high-compliance protocol was done first. The rationale for this was that, if there was any fatigue or losses in force over the duration of the experiment, that the alternation of the order would equalize the results. By chance, the success level of the experiments (see page 52 for inclusion criteria) to this point had been far in favor of those in which the low-compliance protocol was 
performed first, to the point where of these 10 experiments, 8 of them were performed with the low-compliance protocol first, and 2 of them performed with the high-compliance protocol done first. Of the 2 experiments performed with the high-compliance protocol first, the results were in stark contrast to the other 8 . Instead of the additional in-series compliance reducing force output, the relative force output of the high compliance $100 \mathrm{~Hz}$ condition was actually $8 \%$ and $17 \%$ higher than that of the low-compliance condition. Therefore, with this data it was impossible to say whether the reductions in force that were seen were due to shortening-induced force depression due to the additional in-series compliance, or if there was just a loss of force due to fatigue or some other factor that was causing it. Obviously, we would not be able to state with any certainty what the real cause of the effects we were seeing was, and this was a problem that we had to deal with.

We surmised that perhaps the muscle was getting fatigued over the course of the experiment, and if we decreased the total number of contractions performed, this would prove to be less of an issue. As well, in order to confidently state that it was in fact the additional in-series compliance that was causing the reductions in force, and not fatigue over the experiment, I made sure to perform the majority of the experiments with the high-compliance protocol first.

\subsubsection{Stimulation Protocol Version 3}

As mentioned above, the goal with this set of experiments was to minimize any force losses over the course of the experiment due to fatigue, and to ensure that we could confidently state the reason for the losses in force. We made the decision to only look at the highest and lowest activation conditions, $100 \mathrm{~Hz}$ contractions and twitch contractions, and to perform the high-compliance protocol first for most of the experiments. The experimental setup was identical to the previous procedures, but for the stimulation protocol, we only performed twitch 
contractions at reference length $\pm 4 \mathrm{~mm}$ with 1 minute between contractions, and then $100 \mathrm{~Hz}$ contractions at reference length $\pm 4 \mathrm{~mm}$ with 5 minutes between contractions, before changing the in-series attachment and repeating the protocol.

\section{Table 3.4 Relative peak force and optimum length for twitch and $100 \mathrm{~Hz}$ contractions}

\begin{tabular}{|c|c|c|c|c|c|c|}
\hline \multirow[t]{2}{*}{ Condition } & \multicolumn{2}{|c|}{$\underline{\text { Relative Peak Force }}$} & \multicolumn{2}{|c|}{$\underline{\text { Relative Optimum Length }}$} & \multicolumn{2}{|c|}{$\begin{array}{l}\text { Compliance at Reference } \\
\text { Length }\left(\mathrm{mm} \cdot \mathrm{N}^{-1}\right)\end{array}$} \\
\hline & $\begin{array}{c}\text { Low } \\
\text { Compliance }\end{array}$ & $\begin{array}{c}\text { High } \\
\text { Compliance }\end{array}$ & $\begin{array}{c}\text { Low } \\
\text { Compliance }\end{array}$ & $\begin{array}{c}\text { High } \\
\text { Compliance }\end{array}$ & $\begin{array}{c}\text { Low } \\
\text { Compliance }\end{array}$ & $\begin{array}{c}\text { High } \\
\text { Compliance }\end{array}$ \\
\hline Twitch & $0.23 \pm 0.02$ & $0.19 \pm 0.01$ & $1.17 \pm 0.03$ & $1.14 \pm 0.02$ & $0.53 \pm 0.11$ & $0.66 \pm 0.15$ \\
\hline $100 \mathrm{~Hz}$ & $1.00 \pm 0.00$ & $1.03 \pm 0.04$ & $1.00 \pm 0.00$ & $0.92 \pm 0.03$ & $0.35 \pm 0.04$ & $0.55 \pm 0.06$ \\
\hline
\end{tabular}

Peak force and optimum length expressed relative to the $100 \mathrm{~Hz}$ low-compliance condition. Values are mean $\pm \operatorname{SEM}(\mathrm{n}=5)$.

Separate two-way repeated measures ANOVA were done to determine the effect of activation condition and compliance on the relative force output and relative optimum length. There was no significant interaction between activation level and compliance on peak force $(\mathrm{p}=0.094)$, or optimum length $(\mathrm{p}=0.092)$. There was a significant main effect of activation level on force output $(\mathrm{p}<0.001)$, but no effect of compliance condition on force output $(\mathrm{p}=0.938)$. Likewise, there was a significant main effect of activation level on optimum length $(\mathrm{p}=0.004)$, but no effect of additional compliance $(\mathrm{p}=0.087)$. The goal with this protocol was to minimize any unexplained losses in force during the experiment, in order to clearly show the effects that in-series compliance had on force output and optimum length. Unfortunately, it didn't really show much of anything that was actually significant. Of course, the peak force of twitches was less than that of $100 \mathrm{~Hz}$ contractions, and of course the optimum length of twitches was longer than that of $100 \mathrm{~Hz}$ contractions. But these data showed no significant effect of compliance on peak force, which was not what we expected. See Table 3.4 for full results. 
For this data set, 4 experiments were done with the high-compliance protocol first, and one experiment was done with the low-compliance protocol first. Curiously, the one experiment done with the low-compliance protocol first showed that the high-compliance $100 \mathrm{~Hz}$ peak force was $8 \%$ lower than the low compliance condition, while for the other 4 experiments, force in the high compliance condition was on average $7 \%$ higher than the low-compliance condition. While the main effect of compliance was not statistically significant for either peak force or optimum length, the mean values suggest that adding in-series compliance increased force, but decreased optimum length. While Holt and Williams (2018) did suggest that additional compliance would decrease the optimum length via shortening-induced force depression, there was no clear reason for an increase in force due to this additional compliance. The only real explanation was that we were still getting reductions in force output over the course of the experiment due to fatigue or damage to the nerve. Regarding the apparently reduced optimum length with the additional compliance, it was evident in some experiments that the force of the contractions often brought the muscle to the ascending limb or plateau of the force-length curve, but no further. When these data were fitted with a polynomial equation, the result may have been an "artificially" reduced optimum length, since the end of the plateau and the descending limb were absent from the data (see Figure 3.7 below). 


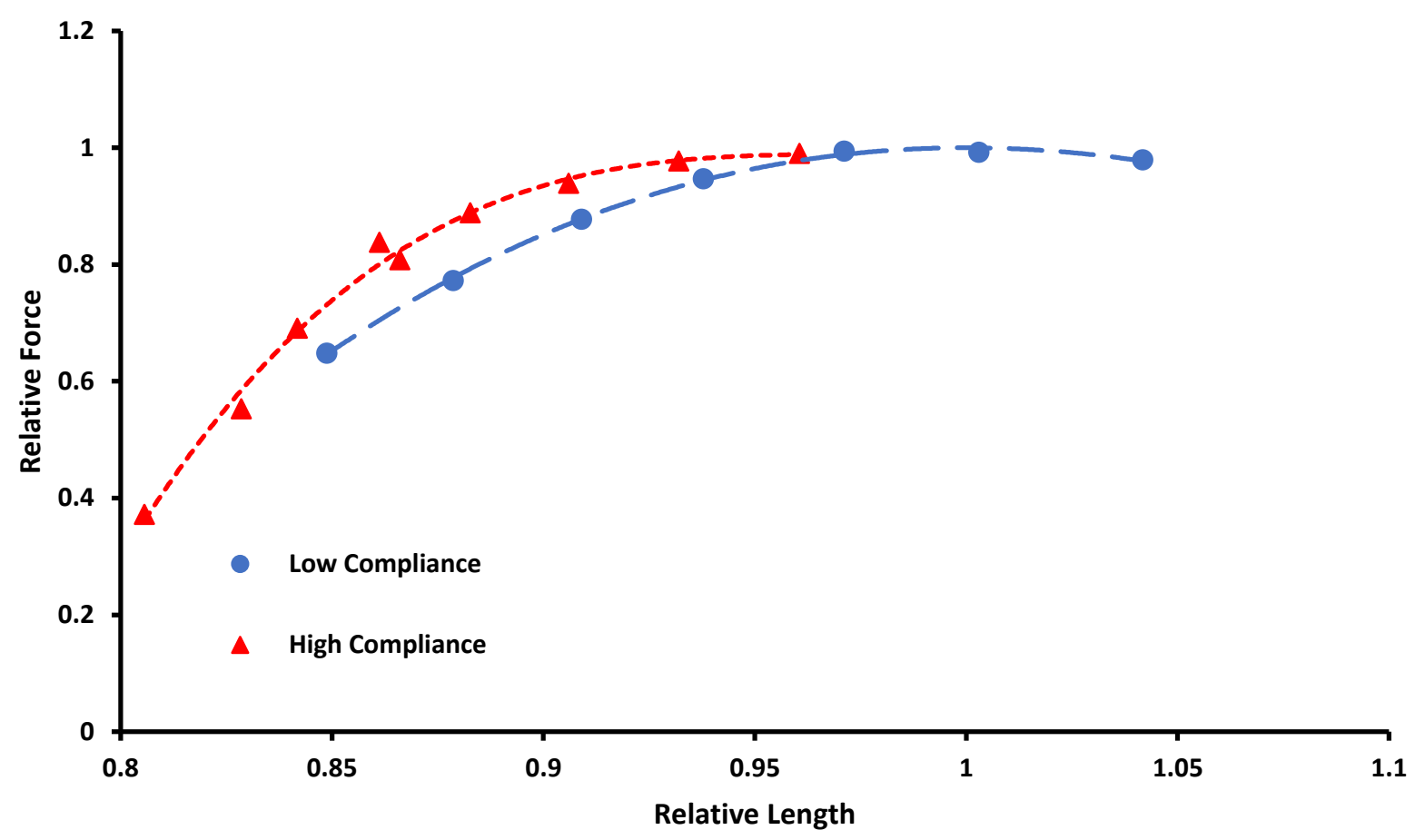

Figure 3.7 Incomplete force-length relationship due to excessive muscle shortening.

Example data from one of the above experiments showing force-length curves for $100 \mathrm{~Hz}$ contractions with high in-series compliance (triangles) and low in-series compliance (circles). Note the slightly increased force of the high-compliance condition, as well as the incomplete curve, which may have artificially skewed the optimum length to shorter lengths when fit with a polynomial equation.

While the earlier experiments showed a reduction in force with the additional in-series compliance, these five experiments seemed to show the opposite trend. The main factor that changed between the two sets of experiments was the order in which the different protocols were performed, and this still seemed like the most likely explanation for this discrepancy. Evidently, we were still having some issues with unexplained losses in force over the course of the experiment. Whether this was fatigue, or whether there was some slight nerve damage that resulted in progressive losses in force with more stimulation, the fact was that it was confounding our results to where we couldn't really say what was causing the reductions in 
force. Since force depression was one of the key things we were interested in, this still posed a problem that needed to be addressed.

\subsection{The Final Approach}

The difficulties in this previous set of experiments led us to our final, and most successful protocol which forms the bulk of the presented data for this thesis. We set out to reduce the potential for fatigue even more than we had already, by shortening the duration of our "highactivation" contractions from $300 \mathrm{~ms} 100 \mathrm{~Hz}$ contractions to $200 \mathrm{~Hz}$ triplet contractions. This would also slightly reduce the force output, hopefully preventing the muscle from "overshortening," such that we could get complete force-length curves to more accurately estimate the optimum length and the peak force that coincided with this length. 


\section{Final Approach}

\subsection{Introduction}

Through much trial and error, we were able to modify our experimental protocol to one that produced consistent results. We solved the issue of force losses over the duration of the experiment by decreasing the number and duration of contractions in the protocol (and likely by me also improving my surgical skills resulting in more consistent crystal placement and less nerve damage). Additionally, to be sure that any force losses over the duration of the experiment were not confounding our results, 6 of the 8 experiments were performed with the highcompliance protocol first, before any fatigue or losses in force would be apparent. We solved the issue of the added in-series compliance being too high by fine-tuning the stiffness and length of the silicon tubing; finally, we solved the issue of the nerve cuff connection quality not allowing us to obtain consistent force-length curves with reduced motor unit recruitment by obtaining a higher quality cuff that maintained a better connectivity with the nerve. Finally, the potential effect that differences in the velocity of shortening between conditions of high and low compliance became a point of interest subsequent to the original proposal, and consequently was included in the analysis. Our final approach will be detailed below.

\subsection{Methods}

\subsubsection{Muscle Preparation}

Female Sprague Dawley rats ( 250-400g) were fed standard rat chow and water ad libitum. All procedures were approved by the University of Calgary Animal Care Committee. The rats $(n=8)$ were anaesthetized with $2 \%$ isoflurane in oxygen and had their left hindlimb surgically prepared for in situ measurement of force of the medial gastrocnemius muscle (MacIntosh, Esau, Holash, \& Fletcher, 2011). An incision was made through the skin from the 
calcaneus to the spine, and the subcutaneous connective tissue was cleared away. The superficial hamstring muscles of the thigh were cut to expose the sciatic nerve. With the aid of a dissecting microscope and microstimulation, nerves innervating any muscle but the medial gastrocnemius were cut, to ensure that the medial gastrocnemius was the only muscle that would exert force upon stimulation. A string was tied around the sciatic nerve proximally, and the nerve was cut above this. The triceps surae muscle was further dissected, and the tendons of the plantaris, soleus, and lateral gastrocnemius were cut from the calcaneus, again to ensure that all measured force would be from the medial gastrocnemius. A silk cord was tied around the Achilles tendon, and the calcaneus was then cut from the rest of the foot, leaving a small piece of the calcaneus attached to the Achilles tendon to prevent the string from slipping off. The foot and lower leg were then amputated by cutting through the tibia about midway along the bone. A metal probe was placed in the open end of the tibia, and a rod was inserted perpendicularly into the femur to stabilize the rat in the apparatus. A single muscle fascicle in the medial gastrocnemius was identified by microstimulation, and piezoelectric crystals (Sonometrics, London ON, Canada) were inserted into each end of the fascicle by creating small pockets with forceps, and the crystal was secured with tissue glue (Vetbond, 3M, St. Paul, MN, USA). The silk cord attached to the Achilles tendon was attached to a force transducer directly, or in combination with a short piece of silicon tubing, and the metal probes inserted into the tibia and femur were secured onto a metal frame to immobilize the rat's leg during the experiment. A stepper motor (Parker Motors, Parker Hannifin Corporation, Charlotte NC) allowed the MTU length to be changed. The rat's skin around its lower limb was pulled up and attached to the metal frame by elastic bands to create a bath that was filled with warm mineral oil, and the muscle was kept at physiological temperature with aid from an infrared heat lamp and tin foil shields. Rectal and oil bath 
temperatures were kept between $36.5^{\circ}$ and $38^{\circ} \mathrm{C}$. The cut sciatic nerve still attached to the medial gastrocnemius was inserted into an electrode cuff connected to a Grass S88 Stimulator (Grass Instruments, Quincy, MA, USA). All stimulation protocols used supramaximal square wave pulses with a $50 \mu$ s pulse width.

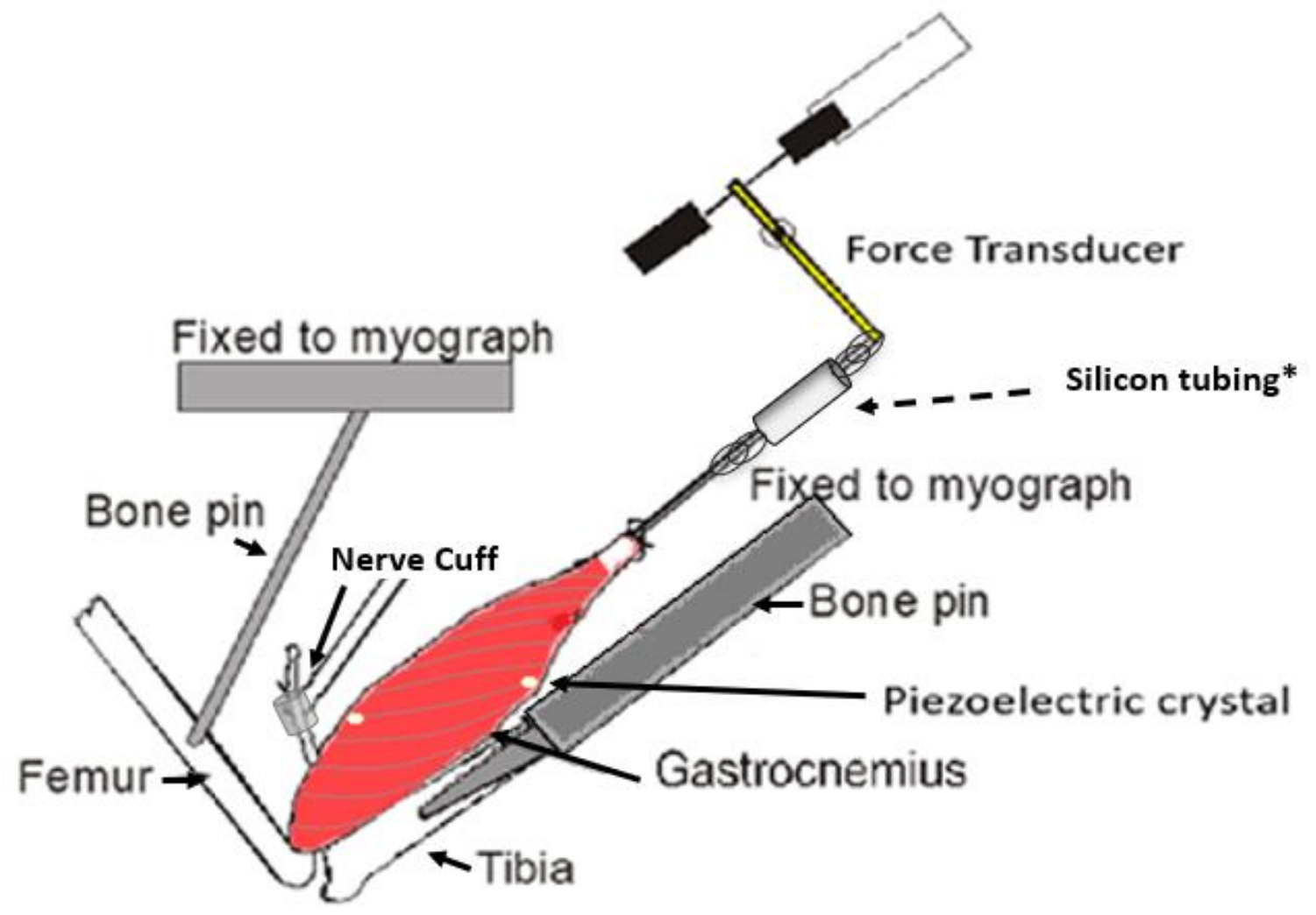

Figure 4.1 In situ muscle preparation.

Adapted from MacIntosh et al (2011). See above text for detailed description.

\subsubsection{Stimulation Protocol}

Stimulation voltage used during the experiments was found by stimulating the nerve with single pulses while decreasing the stimulation voltage. The lowest voltage that resulted in $100 \%$ of twitch force was then doubled for use during the experiment. This supramaximal voltage ensures that all responsive motor units were being recruited. Reference optimum length was then 
found by stimulating with double pulses at $200 \mathrm{~Hz}$ while varying the MTU length in $1 \mathrm{~mm}$ increments. The active force (total force minus resting force) was calculated for each length, and the length that corresponded to the highest active force was used as the "reference length" for each protocol. Twitch force-length curves were obtained first by obtaining twitch contractions at reference length and at $1 \mathrm{~mm}$ intervals $\pm 4 \mathrm{~mm}$ with 60 seconds between contractions. Following this, contractions evoked by 3 stimuli at $200 \mathrm{~Hz}$ were obtained at reference length and $1 \mathrm{~mm}$ intervals $\pm 4 \mathrm{~mm}$, with 5 minutes between contractions in order to minimize any potential for fatigue or potentiation. When this protocol was finished, the in-series attachment was changed to alter the compliance, and the entire protocol was repeated once more on the same muscle. For the final 4 experiments, a third protocol was completed in which the low-compliant attachment was used (no additional silicon tubing), but the stimulation voltage was reduced, such that the triplet force output matched that of the high-compliant condition, in order to determine the effect of reducing voltage as was done by Holt and Azizi (2014), and to compare the effect of the work done at a given force output on the optimum length. HF-LC = triplet contractions in the low compliance condition. $\mathrm{HF}-\mathrm{HC}=$ triplet contractions in the high compliance condition. $\mathrm{LF}-\mathrm{LC}=$ twitch contractions in the low compliant condition. $\mathrm{LF}-\mathrm{HC}=$ twitch contractions in the high compliant condition. $\mathrm{RF}-\mathrm{LC}=$ reduced voltage triplet contractions in the low compliant condition.

\subsubsection{Data Analysis}

All data were collected via a PowerLab data acquisition device and LabChart software (ADI Instruments, Colorado Springs, USA) at a sampling rate of $2000 \mathrm{~Hz}$. Data were then exported to Microsoft Excel (Microsoft Corporation, Redmond, USA) and IBM SPSS (IBM, Armonk, USA), for further analysis. For each contraction, force prior to the contraction (passive 
force), force at the peak of contraction (total force), fascicle length prior to the contraction (preshortening fascicle length), and fascicle length at the peak of contraction (post-shortening length) were recorded. Active force was calculated as recommended by MacIntosh and MacNaughton (2005) other than where explicitly noted. A passive force-length curve was fit with an exponential function of the form $F=a^{*} e^{l * b}$, where $F$ is the passive force, $l$ is the fascicle length at that passive force, and $a$ and $b$ are constants. The active force was calculated by subtracting the passive force estimated at the final post-shortening fascicle length from the total force. Work was calculated as the area under the muscle force-fascicle length graph (e.g. similar to Herzog, Leonard, \& Wu, 2000). Shortening velocity was estimated by finding the average slope of the fascicle length-time curves over a $5 \mathrm{~ms}$ interval at a consistent time from the onset of shortening. Compliance at reference length was calculated by dividing the change in length from peak force and estimated optimum length of the HF-LC condition was calculated by finding the point of maximum force on a $3^{\text {rd }}$ order polynomial fit of the force-length relationship. For each experiment, peak force and optimum length of the other conditions (HF-HC, LF-LC, LF-HC, and RF-LC) were calculated relative to the HF-LC condition in order to be able to compare across different experiments. Relative shift in optimum length was calculated as the twitch optimum length divided by the triplet optimum length for a given compliance condition. To determine if the method of calculation of active force impacted the relative shift in optimum length, active force was also calculated by subtracting from the total force the passive force present prior to the contraction (the traditional method of calculation), rather than the passive force estimated to be present at the peak of contraction (see Section 2.5 for details). Separate two-way repeated measures ANOVA tests were done to determine the effect that activation level and compliance had on the estimated peak force and optimum length, and a three-way repeated measures 
ANOVA was performed to determine the effect that the method of calculation had on optimum length. If significant interaction was seen, a one-way repeated measures ANOVA was done to determine simple main effects. Other measures were compared between conditions of compliance by paired t-tests. Significance level was set at 0.05 . 


\subsection{Results}

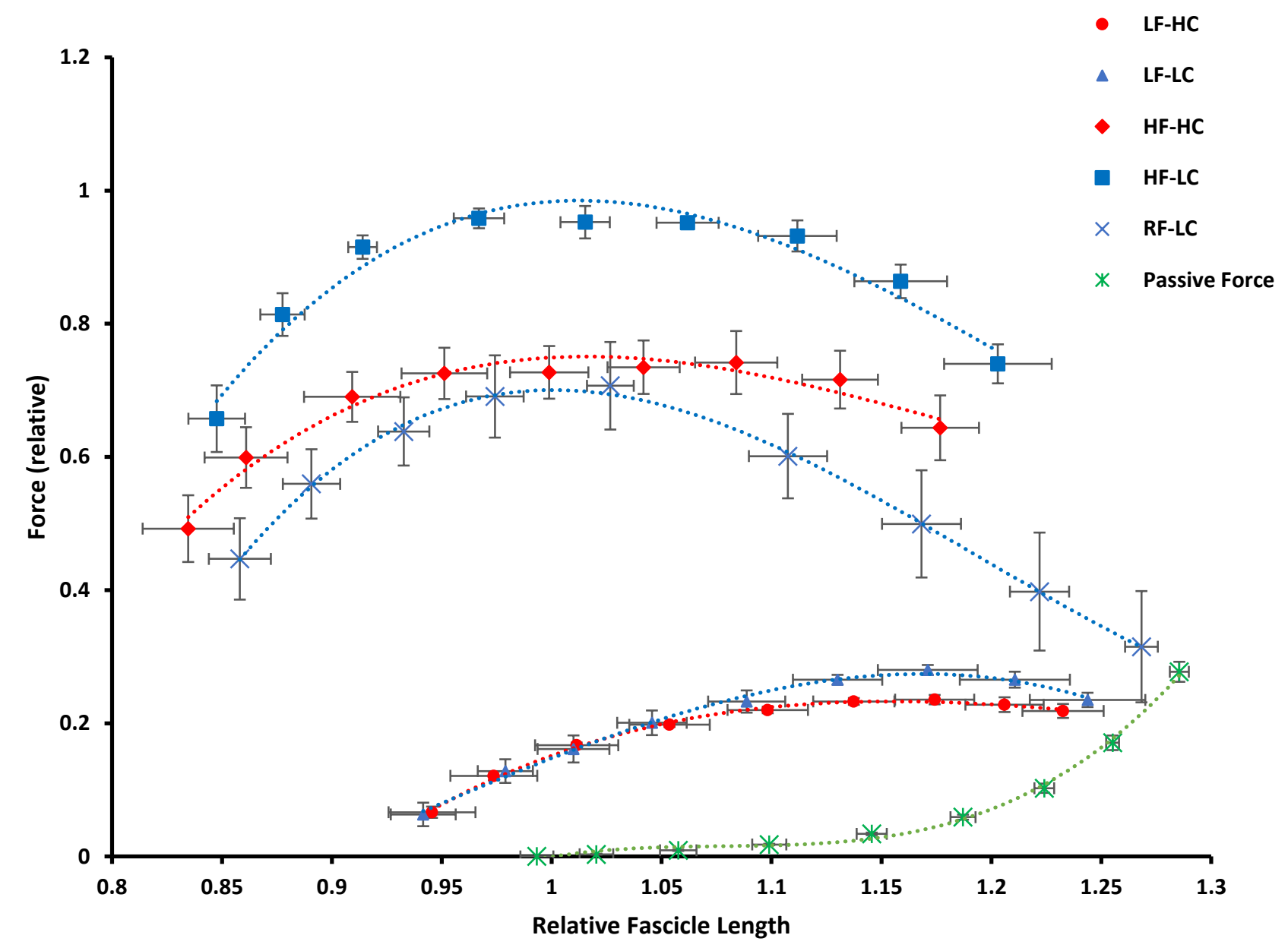

Figure 4.2 The effect of additional in-series compliance on the force-length relationships of triplet and twitch contractions.

Constructed force-length relationships using the mean relative force and relative post-shortening length values for contractions beginning at double pulse reference length and $\pm 4 \mathrm{~mm}$. Data points are mean $\pm \mathrm{SE}(\mathrm{n}=8)$ for HF-LC (blue squares), HF-HC (red diamonds), LF-LC (blue triangles), and LF-HC (red circles) and n=4 for RF-LC (blue x's). F-L relationships are fitted with a $3^{\text {rd }}$ order polynomial function. Although the passive force slightly differed between conditions, the plotted values are the mean initial fascicle length and mean passive force at RL \pm $4 \mathrm{~mm}$ for all conditions grouped together from the last 4 experiments. The mean absolute peak force for the HF-LC condition at RL was $5.32 \pm 1.64 \mathrm{~N}$ (mean $\pm \mathrm{SD}$ ). There was a significant reduction in force between HF-LC and HF-HC conditions, as well as LF-LC and LF-HC conditions. While the $\mathrm{L}_{0}$ of the $\mathrm{LF}$ conditions was significantly increased from the HF conditions, there was no difference in $\mathrm{L}_{0}$ between conditions of compliance at a given level of activation. 
There was no significant interaction between activation level and compliance on the estimated optimum length ( $\mathrm{p}=0.312$ ). Expectedly, the main effect of activation level had a significant effect on the estimated optimum length $(\mathrm{p}<0.001)$. However, the main effect of compliance did not $(\mathrm{p}=0.354)$. There was also no significant difference between the HF-HC optimum length and the force-matched RF-LC optimum length from a paired t-test $(\mathrm{p}=0.550)$. Thus, we did not see any effect of additional in-series compliance on the optimum length. There was a significant interaction between activation level and compliance on the peak force $(\mathrm{p}=0.001)$. Naturally, the peak force was significantly reduced between the triplet and twitch contractions for both compliance conditions. As elucidated in Figure 4.2, however, is that the peak force of the HF-HC condition was significantly smaller than the HF-LC condition $(p=0.001)$. Likewise, the peak force of the LF-HC condition was significantly reduced from that of the LF-LC condition ( $\mathrm{p}=0.001$ ). There was no significant difference in peak force between the HF-HC condition and the force-matched RF-LC condition from a paired t-test $(p=0.765)$. See Table 4.1 for full results. 


\section{Table 4.1 Relative optimum length, peak force, and compliance for different activation and compliance conditions}

\begin{tabular}{|c|c|c|c|}
\hline Condition & $\begin{array}{l}\text { Optimum Length } \\
\text { (relative to HF-LC } \\
\text { condition) }\end{array}$ & $\begin{array}{l}\text { Peak Force (relative } \\
\text { to HF-LC condition) }\end{array}$ & $\begin{array}{l}\text { Compliance at Reference } \\
\text { Length }\left(\mathrm{mm} \cdot \mathrm{N}^{-1}\right)\end{array}$ \\
\hline HF-LC & $1.00 \pm 0.00$ & $1.00 \pm 0.00 *$ & $0.27 \pm 0.03 * \$$ \\
\hline $\mathrm{HF}-\mathrm{HC}$ & $1.02 \pm 0.02$ & $0.75 \pm 0.04 * t$ & $0.49 \pm 0.04 * \$$ \\
\hline RF-LC & $1.02 \pm 0.01$ & $0.71 \pm 0.06^{\ddagger}$ & $0.35 \pm 0.04$ \\
\hline LF-LC & $1.16 \pm 0.02 \%$ & $0.28 \pm 0.01 * t$ & $0.42 \pm 0.04 * t$ \\
\hline LF-HC & $1.16 \pm 0.02$ & $0.24 \pm 0.01 * \hbar$ & $0.64 \pm 0.04 * *$ \\
\hline
\end{tabular}

Values are mean \pm SEM for $n=8$ experiments ( $n=4$ for RF-LC condition). HF-LC $=$ High-force, low-compliance condition, HF-HC = High-force, high compliance condition, RF-LC = Reduced voltage, low-compliance condition, LF-LC = Low-force, low-compliance condition, LF-HC = Low-force, high-compliance condition. The fact that compliance is dependent upon the level of activation (i.e. at a given level of compliance, it varies between high and low-activation) can be explained by a non-linear increase in stiffness as the MTU is stretched to longer lengths, and can be visualized in Figure 4.5. *Denotes significant difference between compliance (LC and HC) conditions. + Denotes significant difference between activation (HF and LF) conditions.

As seen in Table 4.1, there was a significant increase in compliance between LC and HC conditions at both high and low activation. The fact that the compliance decreased at higher activation suggests a non-linear relationship between force and displacement; at higher force outputs, the SEC stretched to a greater length, where the compliance was less, leading to smaller average compliance measurements for those contractions. Assessing the compliance of the additional silicon tubing can be done by assuming that the compliance of a system of in-series arrangements is equal to the sum of the compliances of each individual part, e.g:

$$
C_{\text {system }}=c_{\text {tendon }}+c_{\text {silk }}+c_{\text {silicon }} \ldots
$$

The compliance of the total system without the additional silicon tubing (i.e. Achilles tendon + silk cord) varied between $\sim 0.42$ and $\sim 0.27 \mathrm{~mm} \cdot \mathrm{N}^{-1}$. The additional silicon tubing increased the total system compliance (now Achilles tendon + silk cord + silicon tubing) to $\sim 0.64$ to $\sim 0.49 \mathrm{~mm} \cdot \mathrm{N}^{-1}$. Thus, subtracting the total system compliance with the silicon tubing from the 
total system compliance without the silicon tubing will give the compliance of the silicon tubing.

From the above numbers, it can be calculated that the silicon tubing increased the compliance of the system by $\sim 0.22 \mathrm{~mm} \cdot \mathrm{N}^{-1}$.

Table 4.2 Mean estimated optimum length for different activation and compliance conditions calculated both with and without taking fascicle shortening into account

\begin{tabular}{lccc}
\hline Condition & $\begin{array}{c}\text { Optimum Length } \\
\text { Traditional Method) } \\
(\mathrm{mm})\end{array}$ & $\begin{array}{c}\text { Optimum Length } \\
\text { (Alternative Method) } \\
(\mathrm{mm})\end{array}$ & $\begin{array}{c}\text { Error } \\
\text { (Alternative Optimum Length - } \\
\text { Traditional Optimum Length) } \\
(\mathrm{mm})\end{array}$ \\
\hline HF-LC & $13.81 \pm 0.52$ & $14.06 \pm 0.58$ & $0.25 \pm 0.10$ \\
HF-HC & $13.52 \pm 0.58$ & $14.34 \pm 0.57$ & $0.82 \pm 0.31$ \\
LF-LC & $15.76 \pm 0.45$ & $16.20 \pm 0.47$ & $0.44 \pm 0.06$ \\
LF-HC & $15.51 \pm 0.45$ & $16.17 \pm 0.40$ & $0.66 \pm 0.17$ \\
\hline
\end{tabular}

Values are mean \pm SEM for $n=8$ experiments. No significant interaction was detected between any of the independent variables (method of calculation of active force, activation level or compliance) on the resultant optimum length. There was a significant main effect of the method of calculation $(\mathrm{p}=0.002)$, and activation level $(\mathrm{p}<0.001)$, on the resultant optimum length, however there was no significant main effect of compliance $(\mathrm{p}=0.446)$.

In determining the effect of the method of calculation of active force on optimum length, there was no significant interaction between the method of calculation of active force and either activation level or compliance on the resultant optimum length. However, there was a significant main effect of the method of calculation on the resultant optimum length $(p=0.002)$, whereby the traditional method of calculation consistently underestimated the optimum length compared to the alternative method. There was a trend for the additional compliance to increase the error in calculation at both high- and low activation (means of $0.82 \mathrm{~mm}$ vs $0.25 \mathrm{~mm}$ and $0.66 \mathrm{~mm}$ vs 0.44 
$\mathrm{mm}$, respectively), however the main effect of compliance was not significant. The mean relative shift in optimum length (twitch optimum length divided by triplet optimum length) was not significantly different between low- and high-compliance conditions $(\mathrm{p}=0.308)$. When active force was calculated the traditional way, by subtracting the passive force measured prior to the contraction, there was still no significant difference in mean relative shift between low- and high-compliance conditions ( $\mathrm{p}=0.473$ ). Figure 4.3 shows the data calculated the traditional way, without taking fascicle shortening into account, and the general relationship between conditions is very similar to that seen in Figure 4.2 above. Thus, while the traditional method of calculation did underestimate the optimum length compared to the alternative method, and the additional compliance slightly increased this error, this was not enough to impact the relative shift in optimum length between compliance conditions. 


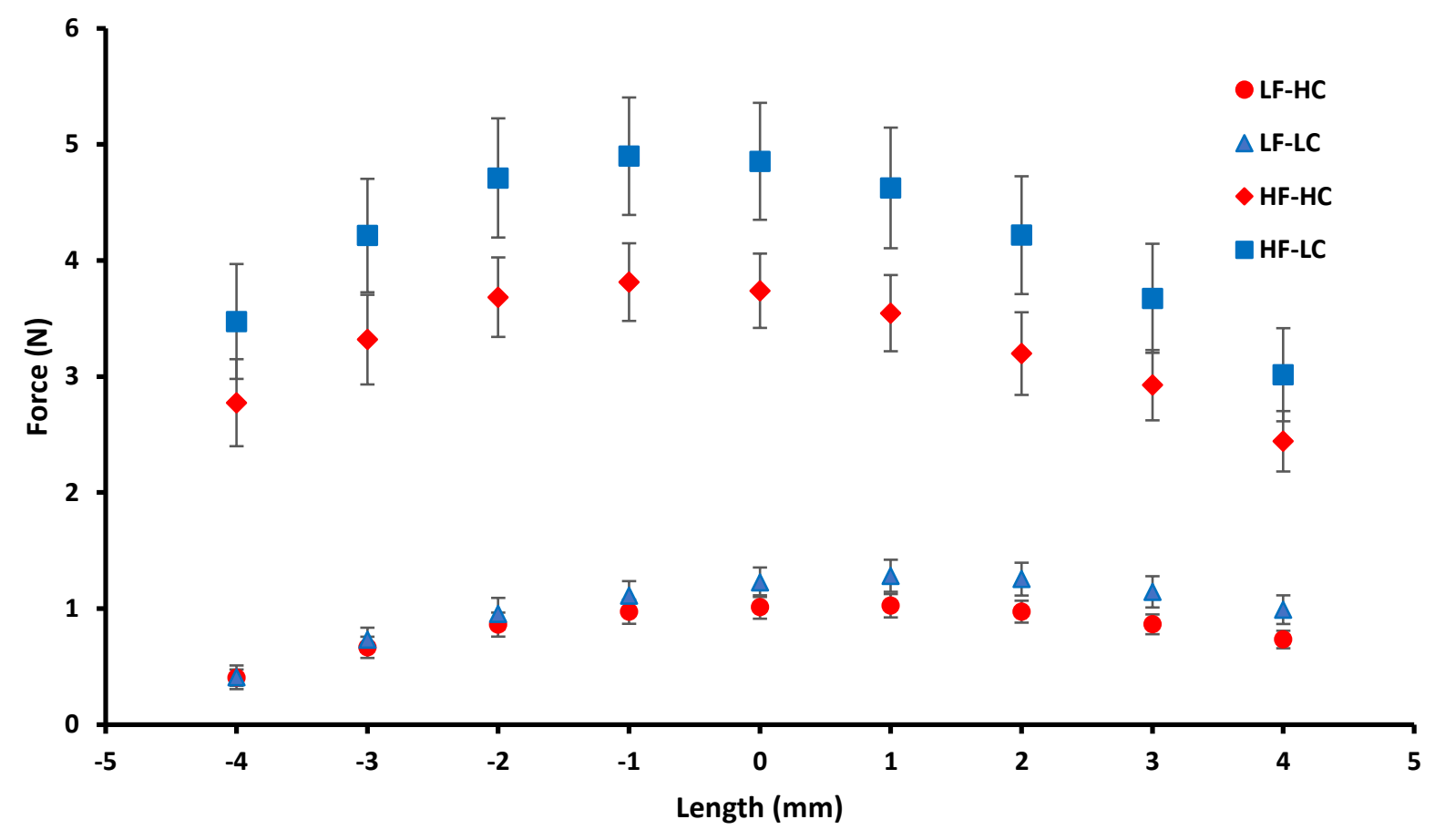

Figure 4.3 Force-length relationship of twitch and triplet contractions calculated the traditional way.

Force-length relationships using the mean force output calculated the traditional way as a function of initial length at reference length $\pm 4 \mathrm{~mm}$ for HF-LC condition (squares), HF-HC (diamonds), LF-LC (triangles), and LF-HC (circles). Data points are mean \pm SE $(n=8)$. Despite not taking into account fascicle shortening or decreases in passive force during a contraction, the general relationships between the conditions appear similar to those seen in Figure 4.2.

In regards to the work done and whether this had any effect on the optimum length - the contractions with additional in-series compliance allowed the muscle to shorten more, and generally increased the amount of work compared to the low-compliance condition (note the difference in work, as well as the difference in final post-shortening length in Figure 4.4 below). However, since the HF-LC contractions also produced more force, the work done was not significantly different between HF-LC and HF-HC conditions (unadjusted $\mathrm{p}=0.094$ ). In the final 4 experiments, when we matched for force output to the HF-HC condition by lowering the stimulation voltage, there was a significant difference in work done at the optimum length 
between the HF-HC condition $($ mean $=4.46 \mathrm{~mJ})$ and the RF-LC condition $($ mean $=2.43 \mathrm{~mJ})$

(unadjusted $\mathrm{p}=0.007$ ). Despite this significant increase in work, there was no difference in optimum length between these two conditions (see Table 4.3 below).

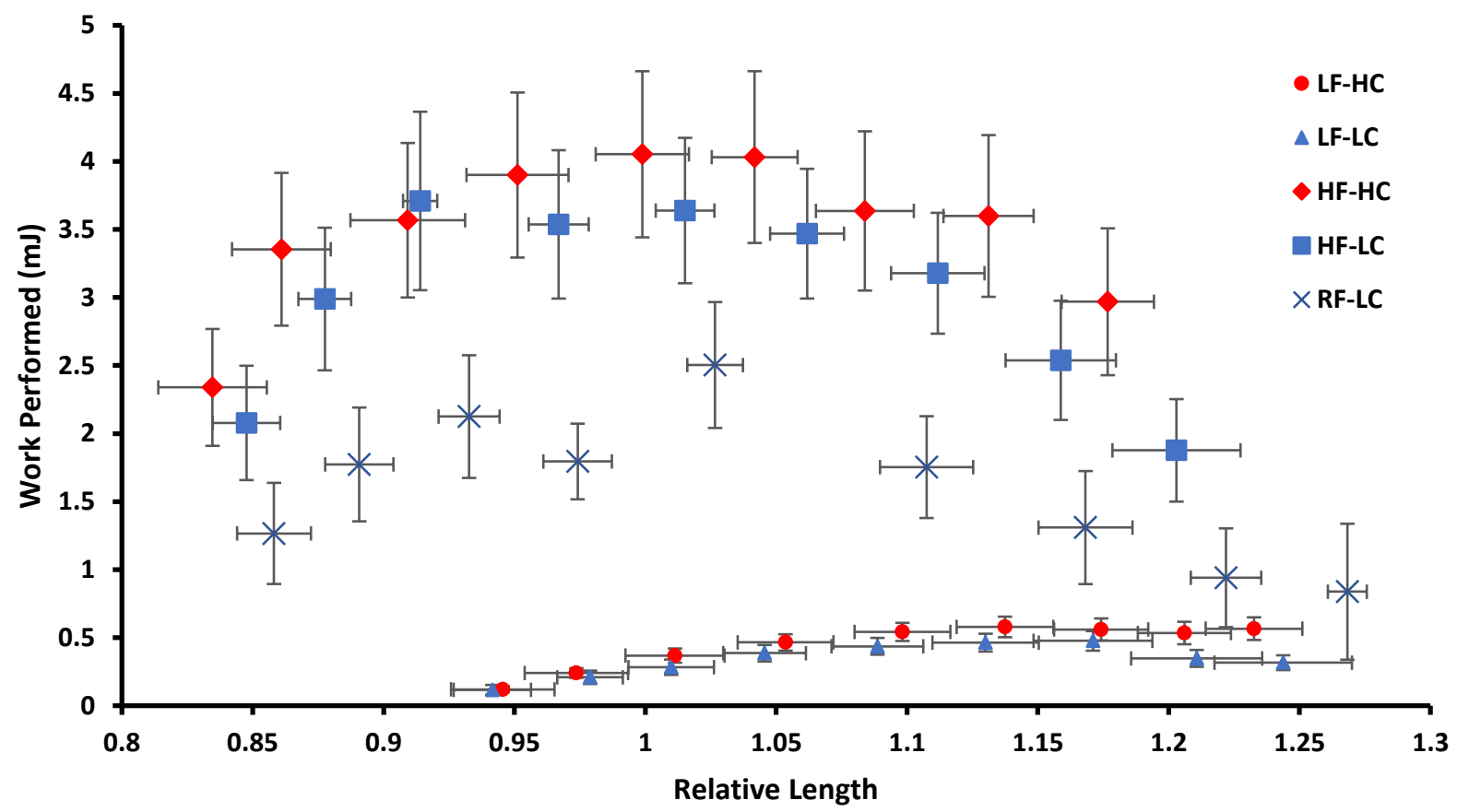

Figure 4.4 Work performed as a function of muscle length.

Constructed relationships using the mean work performed and relative post-shortening length values for contractions beginning at double pulse reference length $\pm 4 \mathrm{~mm}$ for HF-LC condition (blue squares), HF-HC (red diamonds), LF-LC (blue triangles), and LF-HC (red circles) and RFLC (blue x's). Data points are mean \pm SE ( $n=8$ for HF-LC, HF-HC, LF-LC, LF-HC, and $n=4$ for RF-LC). While the additional compliance did allow more work to be performed (compare red data points vs blue data points), the differences in work done at the optimum length were not significant for either HF or LF conditions ( $\mathrm{p}=0.094$ and $\mathrm{p}=0.101$, respectively). When the $\mathrm{LC}$ condition was force-matched to the HF-HC condition (RF-LC), there was significantly less work performed due to the decreased magnitude of shortening. 
Table 4.3 Mean work done at optimum length, mean relative optimum length, and mean relative peak force between $\mathrm{HF}-\mathrm{LC}, \mathrm{HF}-\mathrm{HC}$ and $\mathrm{RF}-\mathrm{LC}$ conditions Work Done at Optimum Relative Peak Force Relative Optimum

\begin{tabular}{lccc} 
Condition & Length $(\mathrm{mJ})$ & at Optimum Length & Length \\
\hline HF-LC & $4.21 \pm 0.33$ & $1.00 \pm 0.00$ & $1.00 \pm 0.00$ \\
HF-HC & $4.46 \pm 0.35$ & $0.76 \pm .07^{*}$ & $1.00 \pm 0.01$ \\
RF-LC & $2.43 \pm 0.51^{* \ddagger}$ & $0.71 \pm 0.06^{*}$ & $1.02 \pm 0.01$
\end{tabular}

Values are mean \pm SEM for $\mathrm{n}=4$ experiments. HF-LC $=$ high force, low compliance, HF-HC $=$ high force, high compliance condition, RF-LC = reduced voltage, low compliance condition. Paired t-tests were done to determine the differences in work performed at optimum length. * Denotes significant difference from the HF-LC condition. $\$$ Denotes significant difference from HF-HC condition.

The difference in force output and magnitude of shortening (related to the amount of work done) can be further visualized in Figure 4.5 and Figure 4.6. Figure 4.5 shows raw tracings of the force and length data from a HF-LC contraction and a HF-HC contraction at reference length. Note the depression of force between the two conditions, as well as the increased magnitude of shortening that occurs with the additional in-series compliance. Figure 4.6 shows example force-fascicle length tracings for HF-LC, HF-HC, LF-LC, and LF-HC contractions at varying lengths. Once again, it can be seen that the low-compliance condition allowed more force, while the high-compliance condition allowed more shortening. What is also evident from Figure 4.6 is the difference in compliance between the conditions, as well as the difference in compliance between fascicle lengths, as noted from the slope of the force-fascicle length tracing. 


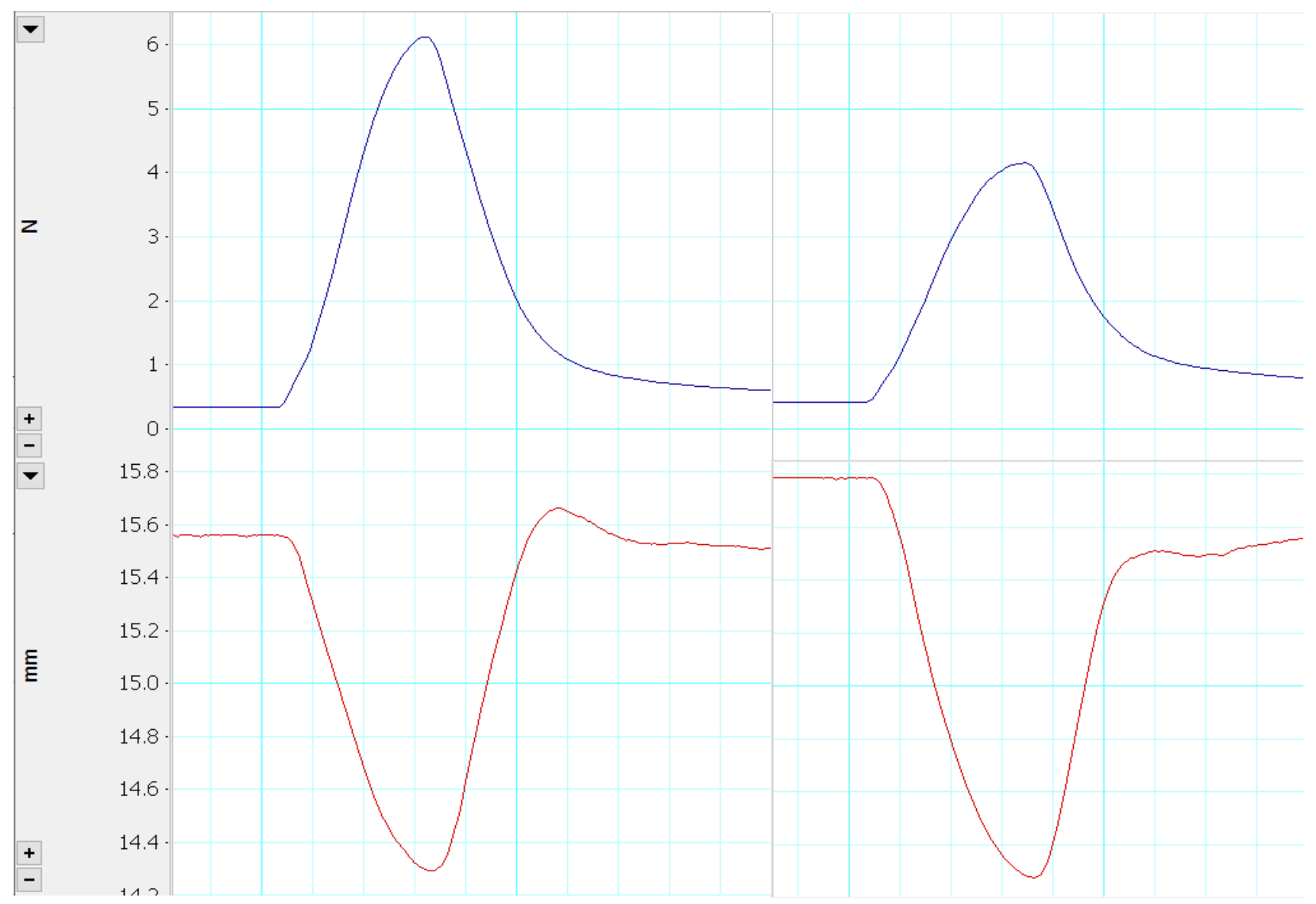

Figure 4.5 Raw data tracings showing the effect of increased compliance on the force output and magnitude of shortening.

Force tracings (blue, top), and fascicle length tracings (red, bottom) for $200 \mathrm{~Hz}$ triple pulse contractions at reference length in the low-compliance condition (left), and the high-compliance condition (right). Note the reduced force output as well as increased length excursion in the highcompliance condition. Shortening velocity was measured as the average slope over $5 \mathrm{~ms}$ of the fascicle length-time curve, taken at a consistent duration from the onset of contraction that corresponded to a straight portion of the fascicle length-time slope. Work was calculated using the force-time and fascicle length-time integrals from the onset of the rise in force until the peak force was reached. 

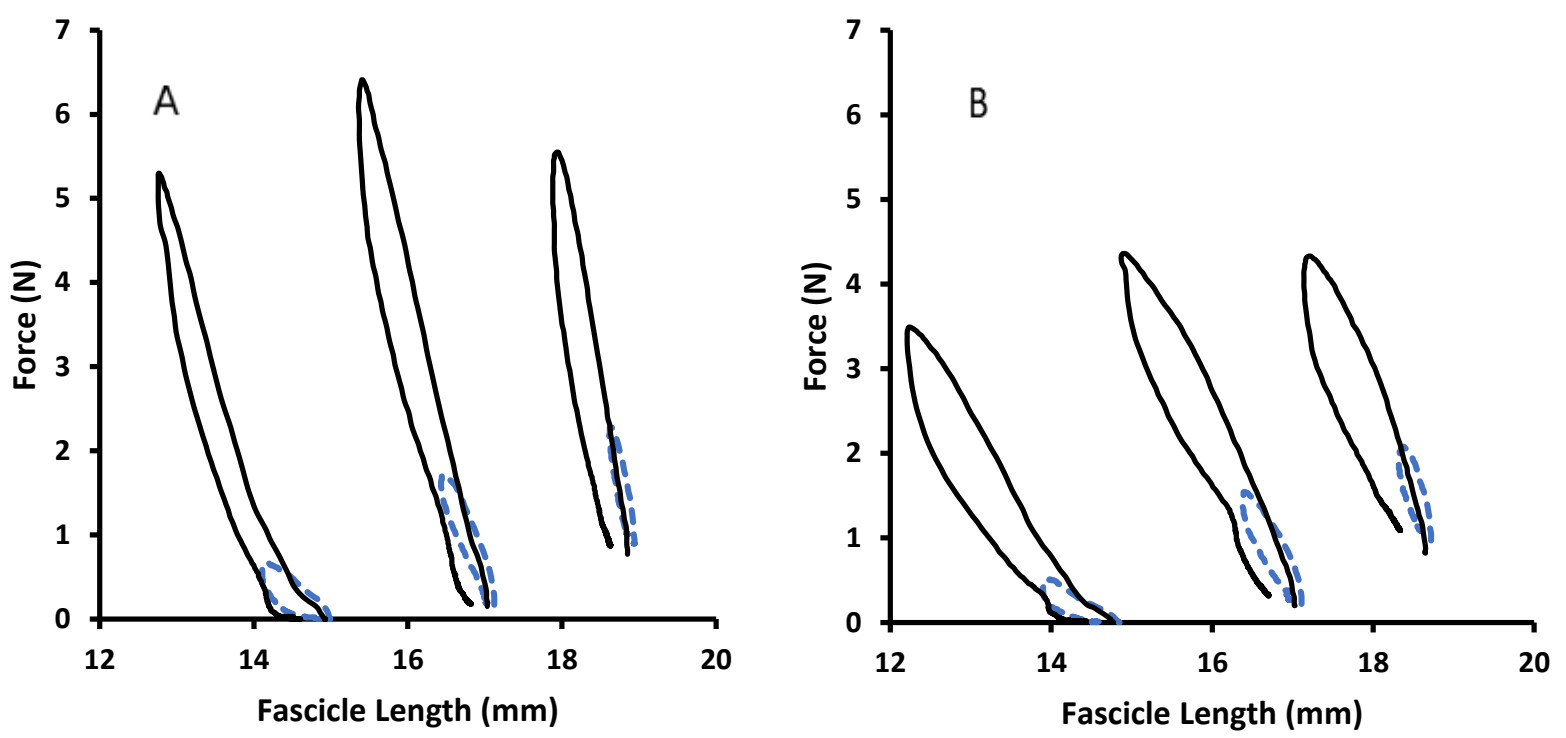

Figure 4.6 Sample force vs fascicle length tracings for triplet and twitch contractions at varying initial fascicle lengths.

Figure 4.5A shows contractions in the low-compliance condition, while Figure 4.5B shows contractions in the high-compliance condition (solid black traces $=$ triplet contractions, dashed blue traces $=$ twitch contractions). From the slope of these tracings, it can be observed that there is a trend for the compliance to increase at shorter lengths (shallower slope), and for the compliance to be greater for twitch contractions compared to triplet contractions at the longer lengths. This is due to the SEC exhibiting a non-linear compliance, whereby compliance will decrease as the SEC is stretched to progressively longer lengths. This effect is particularly evident when the compliance of the connection is high.

As others have done, we tried to correlate the amount of additional work performed with the degree of force depression. As shown in Figure 4.7, while the increased work performed generally corresponded with a decreased force output, there was not a clear relationship between the two factors, suggesting that in our model, the reduction in force output did not appear to be related to the additional work performed per se. 


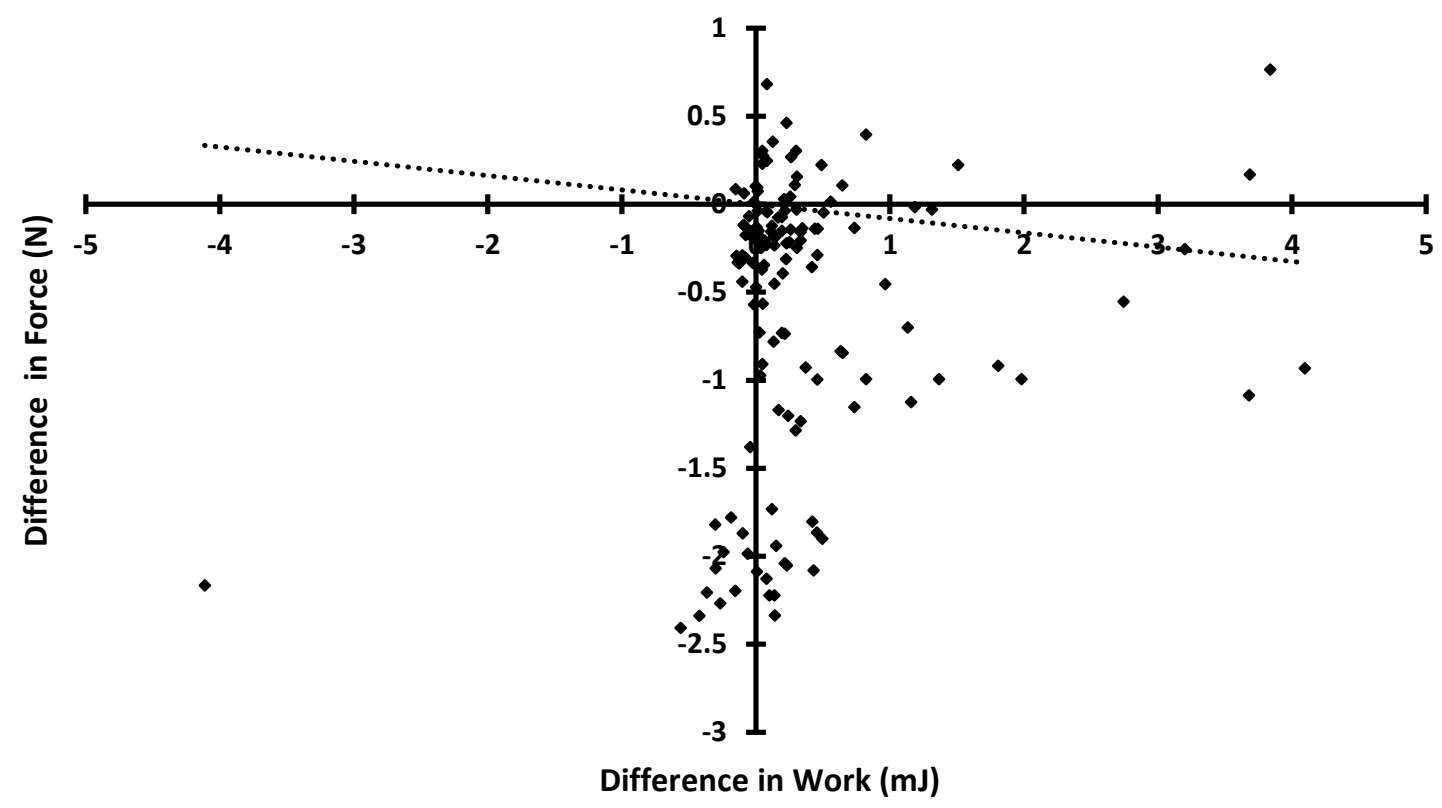

Figure 4.7 Relationship between the difference in force output as a function of the difference in work performed across levels of compliance.

For each experiment $(n=8)$, the difference in work performed and the difference in force produced was calculated between LF-HC and LF-HC contractions, and between HF-HC contractions and HF-LC contractions, at a given starting fascicle length (reference length \pm 4 $\mathrm{mm}$ ). The best fit line was forced through the origin based on the assumption that force depression is due to an increase in work performed, such that no force depression would occur if no additional work was done. The majority of the data points are in the lower right quadrant of the figure, suggesting that the contractions in the high compliance condition performed more work, and produced less force than those in the low-compliance condition, fitting with the notion that additional work performed may have played a role in the force depression seen. However, there is a very large spread in the data, and far from a nearly perfect linear relationship as described by some (e.g. W. Herzog, Leonard, \& Wu, 2000), suggesting that it cannot explain our results on its own.

Related to the amount of work performed, Figure 4.8 shows the magnitude of shortening at $\mathrm{RL} \pm 4 \mathrm{~mm}$ for the HF-LC and HF-HC conditions. The shortening magnitude decreases from short to long MTU lengths, and there is a trend for the additional compliance to allow a greater degree of fascicle shortening at each length. 


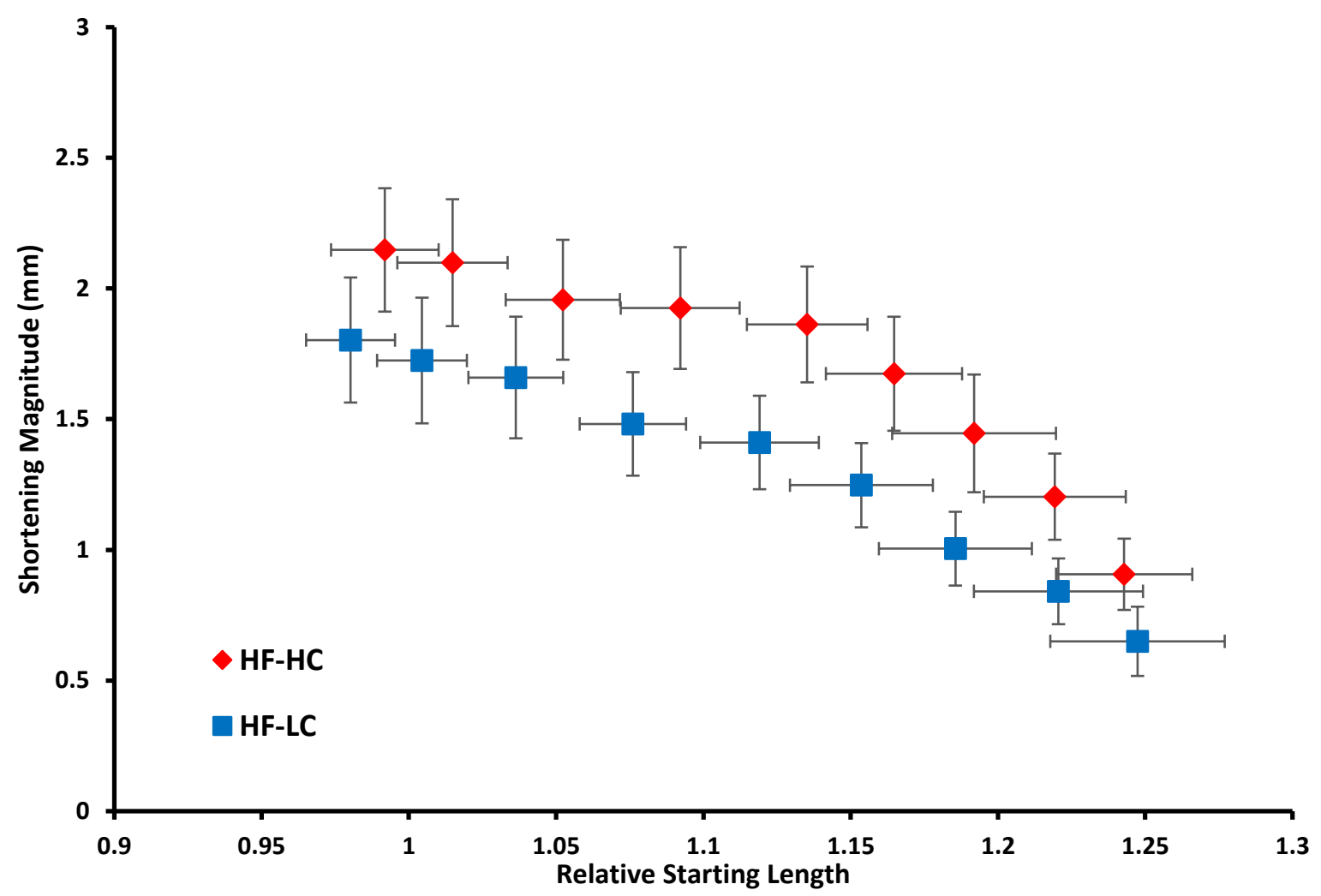

Figure 4.8 The effect of additional in-series compliance on the magnitude of shortening at varying muscle lengths.

Shown is the shortening magnitude as a function of relative starting length for the high-force, high compliance condition (red diamonds), and the high-force, low compliance condition (blue squares). The increased compliance allowed a greater magnitude of shortening, but for both conditions the shortening distance decreased as the MTU length was increased.

The difference in force as a function of the difference in shortening magnitude between conditions of compliance is shown in Figure 4.9 below. Like Figure 4.7, the majority of the data corresponds to an increased shortening magnitude and decreased force output for the $\mathrm{HC}$ condition, however there is still a high degree of variability in the data. 


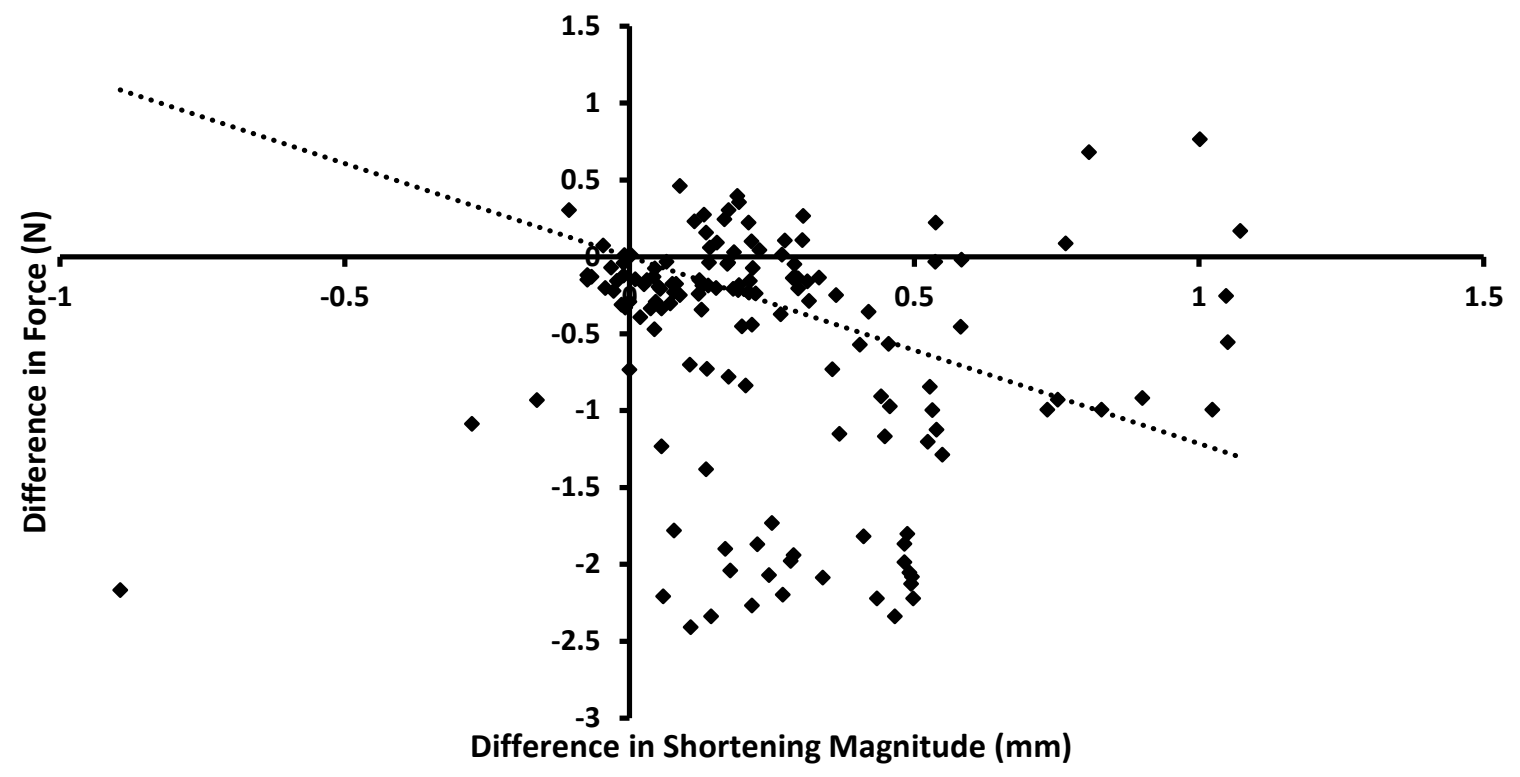

Figure 4.9 Relationship between the difference in force output as a function of the difference in shortening magnitude across levels of compliance.

For each experiment $(n=8)$, the difference in shortening magnitude and the difference in force produced was calculated between LF-HC and LF-HC contractions, and between HF-HC contractions and HF-LC contractions, at a given starting fascicle length (reference length \pm 4 $\mathrm{mm}$ ). As was done with Figure 4.7, the line of best fit was force through the origin.

Shortening velocity was examined in order to determine if this may have been a factor in the reduced force output of the high-compliance condition at a given level of activation. To make this comparison, the shortening velocity corresponding to the highest force contraction (i.e. at optimum length) for each condition was calculated and compared to that of the other compliance condition for that level of activation. While the added in-series compliance did increase the mean shortening velocity at optimum length, this difference was only significant for the twitch contractions $(\mathrm{p}=0.008)$. There was no significant difference in mean shortening velocity at optimum length for the triplet contractions between low- and high compliance $(\mathrm{p}=0.225)$ (see Table 4.4). What was evident, however, was that shortening velocity was always greatest at the shortest lengths (reference length $-4 \mathrm{~mm}$ ) and steadily declines as the muscle was stretched (see 
Figure 4.10 below). As seen in Figure 4.11, this length-dependent decrease in shortening velocity appeared to be linearly related to the decrease in the compliance of MTU-connection system as the MTU was stretched to longer lengths.

Table 4.4 Mean shortening velocity at optimum length for different activation and compliance conditions

\begin{tabular}{ll} 
Condition & Velocity at Optimum Length $\left(\mathrm{mm} \cdot \mathrm{s}^{-1}\right)$ \\
\hline HF-LC & $68.3 \pm 9.6$ \\
HF-HC & $76.4 \pm 8.4$ \\
LF-LC & $27.5 \pm 4.9^{*}$ \\
LF-HC & $37.2 \pm 3.3^{*}$
\end{tabular}

Values are mean \pm SEM for $\mathrm{n}=8$ experiments. HF-LC $=$ High-force, low-compliance condition, HF-HC = High-force, high compliance condition, LF-LC = Low-force, low-compliance condition, LF-HC = Low-force, high-compliance condition. Paired t-tests were done to determine the differences in shortening velocity at optimum length at a given level of activation. * Denotes a significant difference between low- and high-compliance conditions at a given level of activation. 


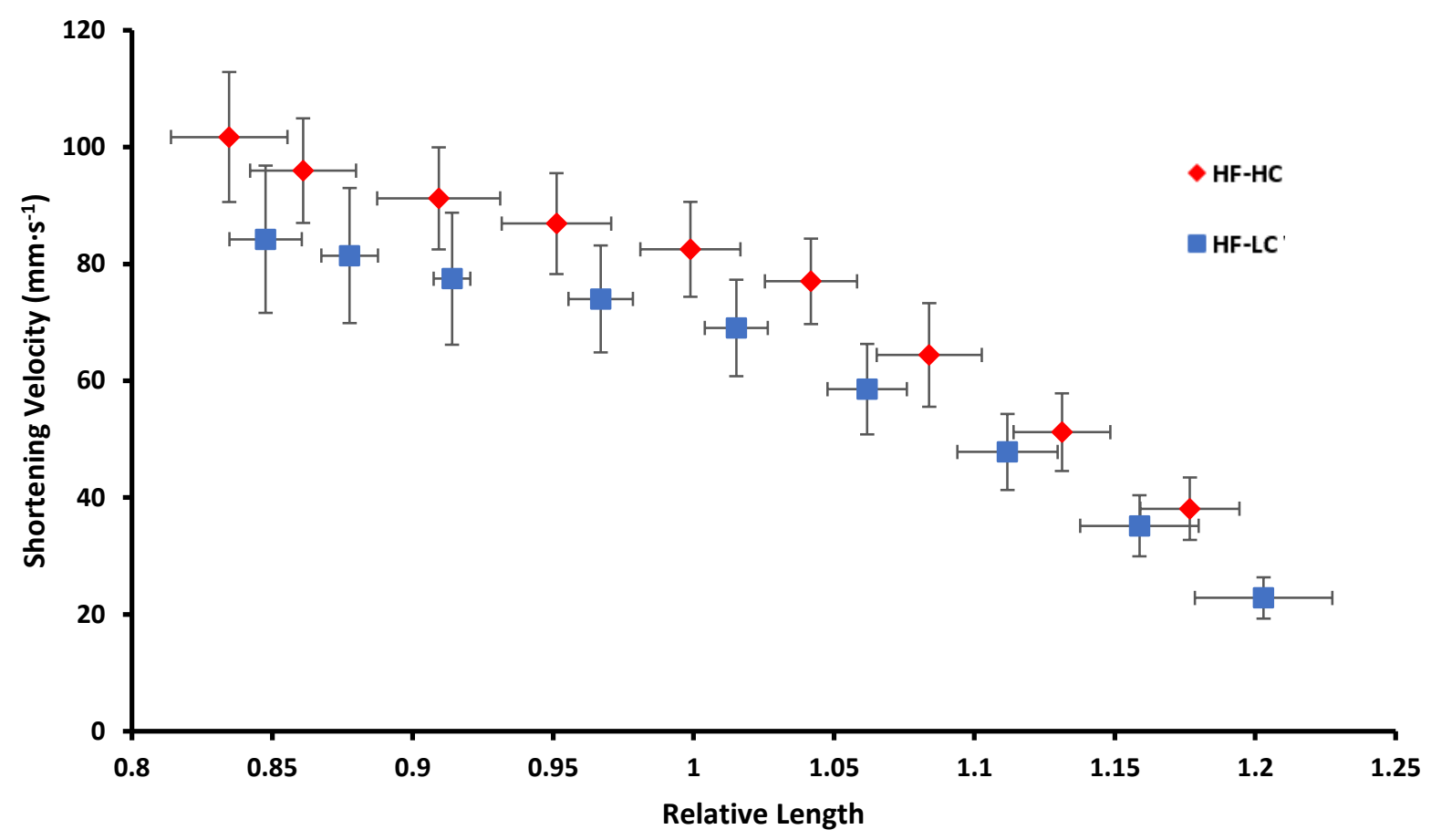

\section{Figure 4.10 The effect of additional in-series compliance on the velocity of shortening at varying muscle lengths.}

Constructed velocity-length relationships using the mean shortening velocity and mean relative post-shortening length values for contractions at double pulse reference length $\pm 4 \mathrm{~mm}$ for HFLC condition (squares), HF-HC (diamonds). Data points are mean $\pm \mathrm{SE}(\mathrm{n}=8)$. The shortening velocity decreases as the length increases, likely due in part to the reciprocal change in compliance over the same length range (see Figure 4.11). While the high-compliance condition tended to have slightly higher shortening velocities at all lengths, the difference at optimum length was not significant for the triplet contractions. 


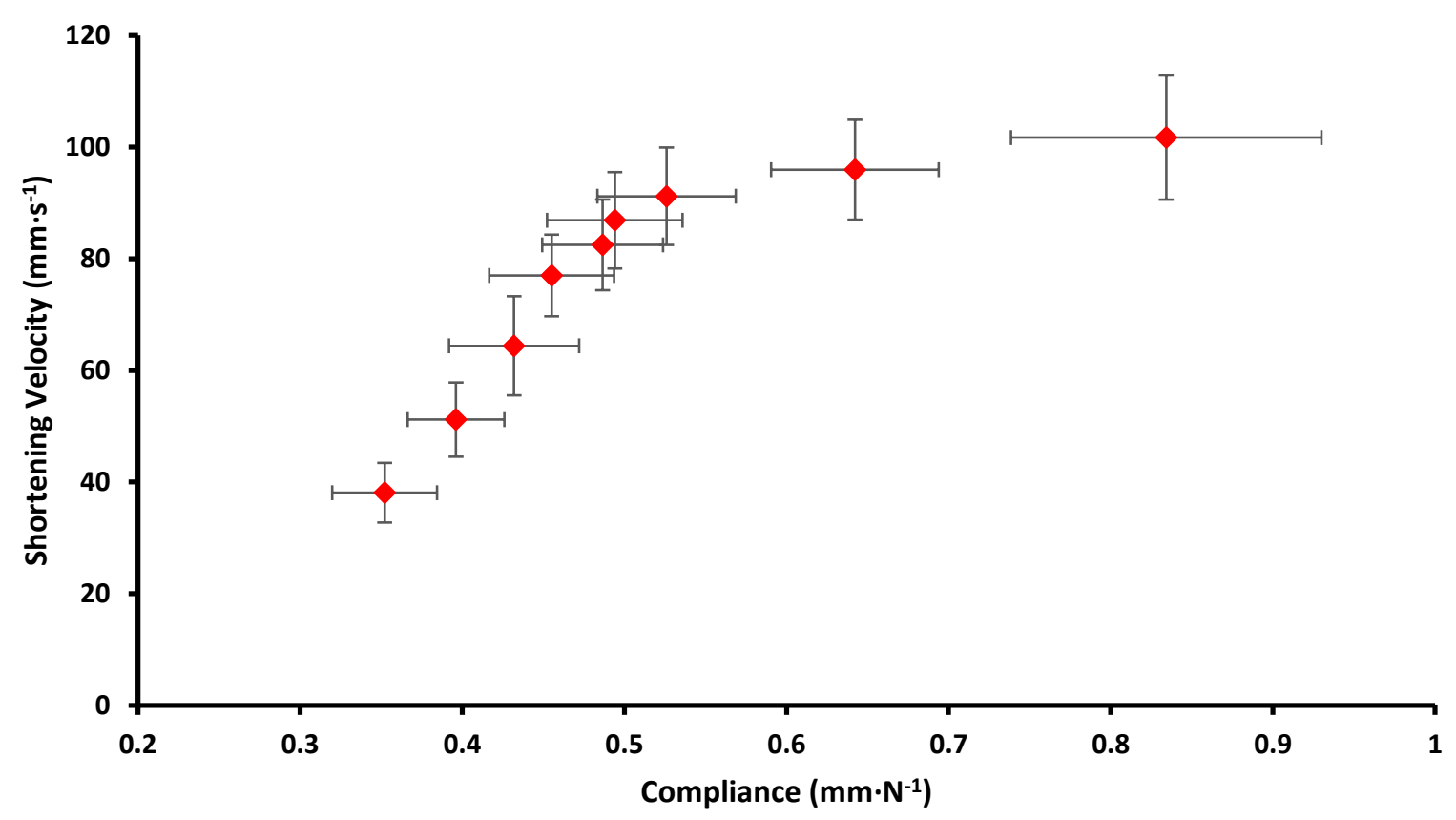

\section{Figure 4.11 The relationship between shortening velocity and compliance at} varying muscle lengths.

Data points are mean $\pm \mathrm{SE}(\mathrm{n}=8)$ at double pulse reference length $\pm 4 \mathrm{~mm}$ for the HF-HC contractions. For most of the curve there is a linear relationship between compliance and shortening velocity. At very high compliances (corresponding to the shortest lengths), the shortening velocity levels out, likely because the muscle is at slack length and so the resistance to shortening stays fairly constant at these short lengths.

As with the effect of additional work performed, we tried to correlate the increased velocity of shortening to the magnitude of force depression to more closely examine the effect that shortening velocity had on the resultant force output. Similar to the effect of additional work, there was a rough correspondence between the increased shortening velocity and the decreased force output, indicating that it may have played a role in the reduction of force observed (see Figure 4.11 below). 


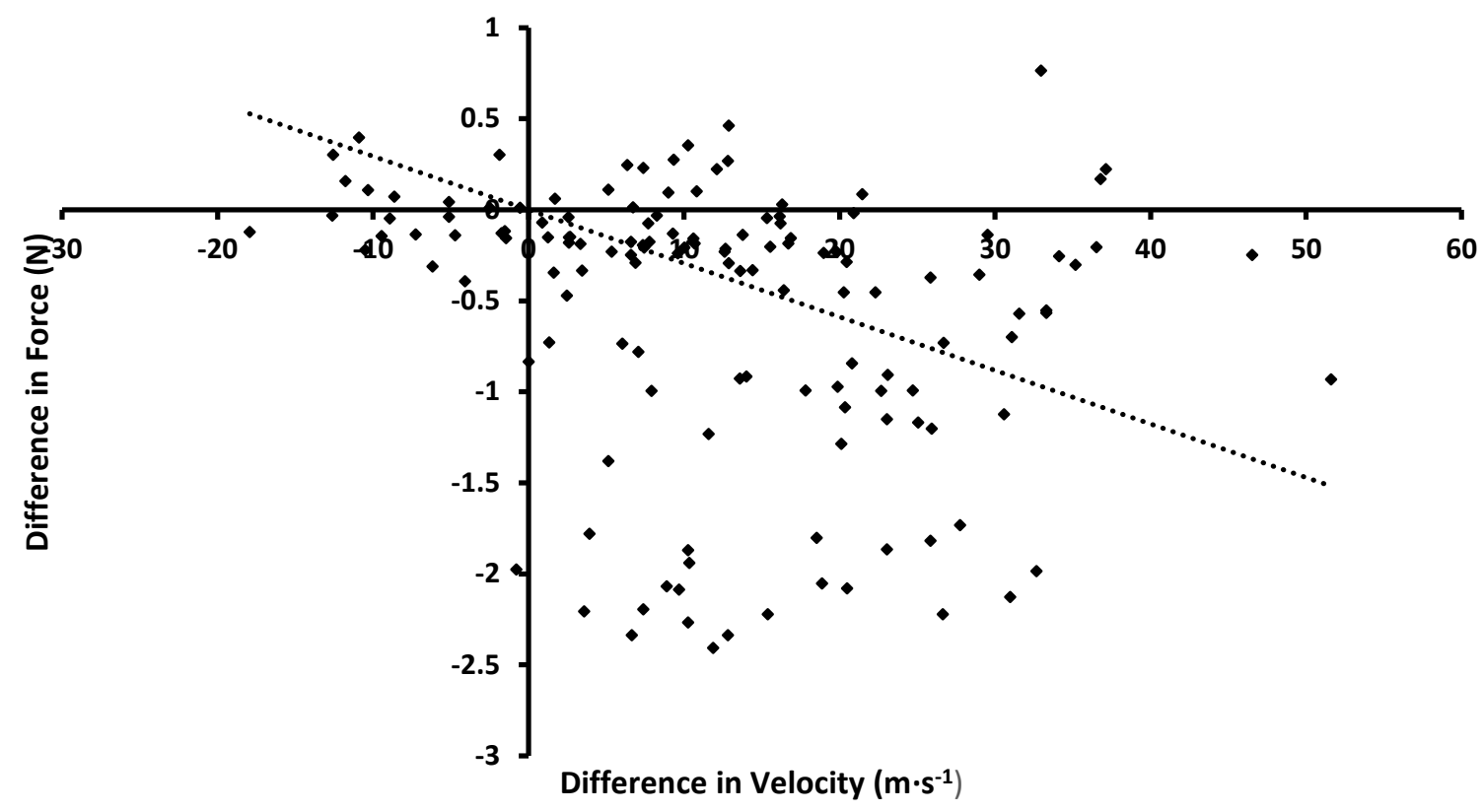

Figure 4.12 Relationship between the difference in force output as a function of the difference in shortening velocity across levels of compliance.

For each experiment $(\mathrm{n}=8)$, the difference in shortening velocity and the difference in force produced was calculated between LF-HC and LF-HC contractions, and between HF-HC contractions and HF-LC contractions, at a given starting fascicle length (reference length \pm 4 $\mathrm{mm}$ ). As with Figure 4.7, the best fit line was forced through the origin based on the assumption that the reduction in force is due to the increased shortening velocity, and no reduction would be seen with no increase in shortening velocity. The majority of the data points are in the lower right quadrant of the figure, showing that the contractions in the high compliance condition had a higher velocity of shortening, and produced less force than those in the low-compliance condition, suggesting that this increased shortening velocity may have been a factor in the reductions in force that we observed. Similar to Figure 4.7, however, the variability in the data is quite high, making it difficult to infer cause and effect relationships.

\subsection{Discussion}

We aimed to determine if additional in-series compliance would impact the force-length relationship in both low- and high-activation conditions. We found that the added compliance significantly reduced the peak force output of both triplets and twitches, but did not impact the optimum length for a given level of activation. We also found that the relative shift in optimum length (twitch optimum length divided by triplet optimum length) was not different between the 
low- and high-compliant conditions, nor did it differ depending on how active force was

calculated despite the method of calculation having a significant, albeit small, effect on the mean optimum length. Based on our findings, we can conclude a few general things. Firstly, we observed that additional in-series compliance significantly reduced force output at both high and low levels of activation. Secondly, despite this reduction in force, we observed no change in optimum length due to this change in compliance at a given level of activation. What seems clear is that, despite the reduction in force output, the post-shortening fascicle length that corresponds to the highest force at any given level of activation does not seem to change, regardless of how much the muscle shortens. At these post-shortening lengths, there are presumably less crossbridges attached, however the degree of overlap is still the chief determinant of the relative force output. At lower levels of activation this still holds true, causing the relative shift in optimum length to also stays constant.

This is at odds with the results of Holt and Williams (2018), who reported a significantly greater relative shift in the more compliant condition compared to the less compliant condition (twitch optimum length/tetanic optimum length of $\sim 1.4$ in the high-compliance condition versus $\sim 1.1$ for the low-compliance condition). They postulated that shortening-induced depression may cause the optimum length of the higher force contractions to shift to the left, increasing this relative difference in optimal length. When we force-matched the HF-HC condition by lowering the stimulation voltage, the work done with the added in-series compliance was significantly greater than in the reduced voltage condition. If force depression is proportional to the work done by the muscle, then one would expect that this high-compliance condition would have more cross-bridge inhibition than the less compliant, but submaximally recruited, condition. Still, there was no difference in optimum length between the groups, leading us to believe that force 
depression does not impact optimum length, or if it does, it is only by a small amount and our measurements were not sensitive enough to observe it.

It should be considered that, in a fixed-end contraction against in-series compliance, the correlation between the force reduction and the work performed is perhaps not as strong as in the more "unnatural" experiments typically exploring shortening-induced force depression. Herzog et al. (2000) studied shortening-induced force depression in cat soleus, and reported that variations in mechanical work could explain almost all of the variance in force depression following shortening. Comparatively, Figure 4.7 shows our results in which the difference in work performed only vaguely related to the difference in force output. In the first case, (if this is in fact the correct mechanism for force depression), the actin filament is maximally strained before any shortening occurs. Upon shortening, it makes sense then that the higher the force, and the longer the distance (whose product is the work performed) will result in the most disruption to new potential cross-bridge binding sites. In our case, the actin filament is not maximally strained before shortening occurs, but theoretically would increase in strain as force rises and the muscle shortens. Additionally, the degree of force depression depends on the time at which the force is measured after the shortening period. Typically, in experiments designed for measuring force depression, this is measured when the force returns to a steady state. In our case, the contractions were effectively still "dynamic" contractions, and this may have influenced the relationship between work and force depression. One other consideration in interpreting our results shown in Figure 4.7 and Figure 4.10 is that contractions were compared between conditions of compliance at a given starting length. As we have seen, the additional in-series compliance allowed slightly greater shortening upon activation, such that the final lengths of the contractions being compared were presumably not identical. This would affect the strength of the 
relationship between additional work performed and reduction in force output, but it is difficult to estimate to what degree. In any case, while cross-bridge inhibition upon shortening still seems reasonable in our experimental setup, it may not be quite as simple as merely corresponding to the overall work performed.

We expected that the method of calculation of active force would impact the resultant shift in optimum length, as we assumed that the traditional method of subtracting the passive force present prior to the contraction would underestimate the triplet contractions relatively more than the twitch contractions, thus increasing the relative shift in optimum length between the two. This did not prove to be the case. While the traditional method of calculation did result in a significant, albeit small, underestimation in optimum length, and this underestimation was slightly greater with the additional compliance, the level of activation did not impact this error. Thus, the relative shift remained similar irrespective of how active force was calculated. This was somewhat surprising, as we figured that a higher force contraction would shorten more, leading to a larger error in active force calculation for the triplet contractions compared to the twitch contractions. While the higher force contractions certainly did shorten more, the key here is that the optimum length of these contractions occurs at a fairly flat part of the passive force curve. Alternatively, while the twitch contractions shortened less, the twitch optimum length occurs at a steeper part of the passive force curve. Thus, the error in calculation (the force difference on the passive force curve at initial fascicle length and the length after shortening) remained similar between the triplet and twitch contractions, and was proportional to the additional in-series compliance. Thus, this method of calculating active force was not a factor in the relative shift in optimum length. To determine whether or not the method of calculation would have a greater effect on the relative shift if perhaps there was a steeper passive force curve 
than our preparation had, we modeled hypothetical force-length relationships manipulating the passive force curve and the compliance of the system (see Figure 4.13 below). In this model, it was again shown that the method of calculation should not increase the relative shift in optimum length between low- and high-activation. As passive force increases, so too does the relative underestimation in optimum length when subtracting the passive force present prior to shortening (compare figures 4.10B and 4.10D with 4.10A and 4.10C). Similarly, as the compliance of the system increases, a greater underestimation in optimum length is again observed. In both cases, there will be a larger difference between the passive force prior to any shortening and the passive force present at the peak of contraction, widening the error in calculation. Importantly, however, because the passive force curve is an exponential relationship, the curve will always be steeper at longer lengths, nearer the twitch optimum length. Thus, as mentioned previously, even though the shortening magnitude is reduced for twitch contractions, the change in passive force (i.e. the potential error) will be similar to the higher activation contractions due to the increased slope at these critical lengths. This is to say that, unless the shape of the passive force curve is more linear in nature, the method of calculating active force is unlikely to explain any unusual activation-dependent shifts in optimum length such as those reported by Holt and Azizi (2014). 

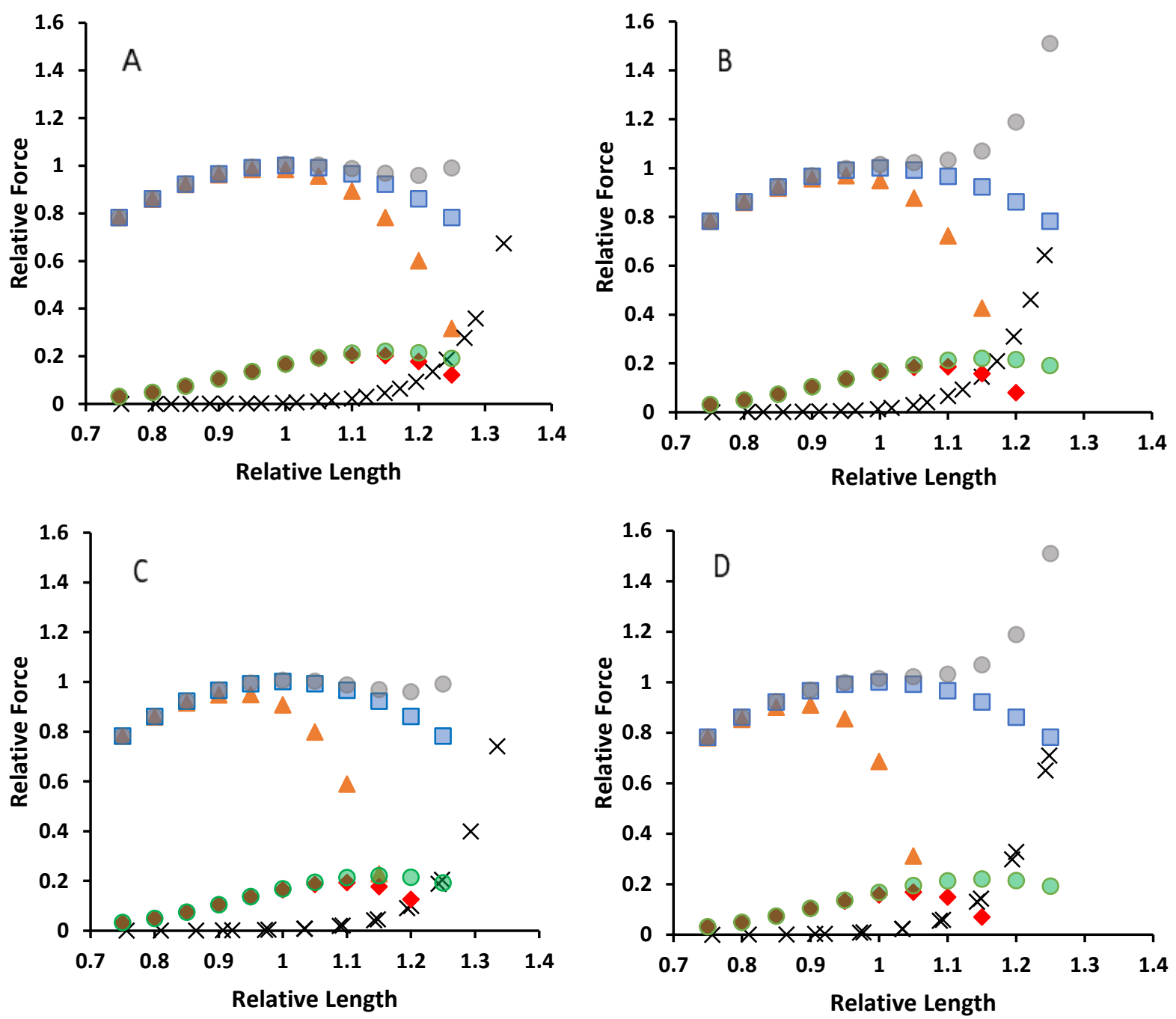

Figure 4.13 The effect of increased passive force and increased compliance on the potential error in calculation of active force.

Hypothetical force-length curves for both high- and low-activation contractions, with active force calculated both the traditional way (subtracting the passive force prior to the contraction) and the alternative way (subtracting the passive force estimated at the post-shortening length). Figures $\mathrm{A}$ and $\mathrm{C}$ show curves with a more gradual passive force curve compared to Figures $\mathrm{B}$ and D. Likewise, figures $C$ and D show curves with the compliance of the system twice that of figures $\mathrm{A}$ and $\mathrm{B}$. Force and fascicle length are reflected relative to the high-activation condition, calculated in the alternative manner. Black x's - passive force. Grey circles - high activation, total force. Blue squares - high activation, alternative method of calculating active force. Orange triangles - high activation, traditional method of calculating active force. Green circles - low activation, alternative method of calculating active force. Red triangles - low activation, traditional method of calculating active force. It can be seen that both increased passive force, as well as increased compliance, can cause underestimation of optimum length to a greater degree when calculating active force in the traditional way (compare the estimated optimum lengths in table 4). However, since both the high- and low-activation conditions are similarly affected by the error in calculation, the relative shift does not increase. 
Table 4.5 Relative optimum length values from Figure 4.13, comparing the effect of the method of calculation of active force on the optimum length while manipulating passive force and compliance

\begin{tabular}{lcccccc}
\hline Figure & \multicolumn{2}{c}{$\begin{array}{c}\text { High Activation } \\
\text { Optimum Length } \\
\text { Alternative }\end{array}$} & $\begin{array}{c}\text { Traditional Activation } \\
\text { Optimum Length }\end{array}$ & $\begin{array}{c}\text { Relative Shift } \\
\text { (Low Activation } \mathrm{L}_{0} / \mathrm{High} \\
\text { Activation } \mathrm{L}_{0} \text { ) }\end{array}$ \\
\hline $\begin{array}{l}\text { A - (lower passive force, } \\
\text { lower compliance) }\end{array}$ & 1.00 & 0.98 & 1.16 & 1.11 & 1.16 & 1.13 \\
$\begin{array}{l}\text { B - (higher passive force, } \\
\text { lower compliance) }\end{array}$ & 1.00 & 0.95 & 1.16 & 1.07 & 1.16 & 1.13 \\
$\begin{array}{l}\text { C - (lower passive force, } \\
\text { higher compliance) }\end{array}$ & 1.00 & 0.94 & 1.16 & 1.09 & 1.16 & 1.16 \\
$\begin{array}{l}\text { D - (higher passive } \\
\text { force, higher compliance) }\end{array}$ & 1.00 & 0.89 & 1.16 & 1.03 & 1.16 & 1.16 \\
\hline
\end{tabular}

Data are calculated from $3^{\text {rd }}$ order polynomial fits of each of the curves in Figure 4.13. From these hypothetical data, one can see that increasing the passive force, as well as increasing the compliance, will tend to further underestimate the optimum length of both high- and lowactivation contractions when calculated in the traditional way. Nonetheless, since both high- and low-activation optimum lengths are similarly affected, the relative shift is constant.

To this point, we have found that shortening induced force depression via additional inseries compliance cannot explain what was observed by Holt and Williams (2018), nor can the error created by inappropriately calculating the active force. We found that increased in-series compliance significantly reduced force output but did not impact the length at which force was greatest. What isn't so clear from our data, however, is what exactly is causing the reduction in force output with the added in-series compliance. In a more tightly controlled experimental protocol, it is much easier to determine cause-and-effect, since only one variable is changing at a time. In our model, however, there are many variables changing at once; fascicle length, series compliance, magnitude of muscle shortening, shortening velocity, etc. In our protocol, the 
contractions with the additional in-series compliance not only shortened more, but also shortened at a higher velocity; both factors that may reduce the resultant force output by different mechanisms - one via shortening-induced force depression, and the other via the effects of the force-velocity relationship. If we were comparing isometric tetanic contractions at varying degrees of activation, then shortening velocity would not pose a potential confounding factor, as we might assume that the duration of the stimulus would be sufficient such that the $\mathrm{Ca}^{2+}$ transients would not rapidly decline before or during the rise in force, which may serve to limit the peak force attained. In our protocol, however, the contractions were very brief as we used only twitches and triplet stimulations in order to minimize fatigue. In these brief contractions, the force would rise in response to the stimulation, and then fall shortly after due to the brevity of the stimulus. At reference length, the time to peak tension of the twitches was $\sim 15 \mathrm{~ms}$. The triplet contractions' force output and time to peak tension is higher due to summation, but the time to peak tension for the triplet contractions was still only about double that of the twitches. If during the rise in force, the muscle is shortening at a higher velocity, perhaps it wouldn't reach as high of a force output before the force begins to decline, thus reducing the peak force output. Indeed, Mayfield, Cresswell, \& Lichtwark (2016) explored the effects of a reduced effective SEC compliance on twitch and doublet contractile responses, and concluded that "modest reductions in fascicle shortening amplitude and velocity owing to a reduction in effective series compliance were sufficient to substantially improve contractile performance," and that "the degree of active shortening afforded by a compliant tendon may be capable of depressing the force-generating potential of consecutive stimuli in a history-dependent manner." Additionally, they proposed that, rather than force-length properties being responsible, "more favourable shortening velocities were primarily responsible for the improvement in twitch performance." 
This increase in velocity afforded by the added in-series compliance may have been a factor in the reduction of force that we observed. However, considering that the difference in velocity between conditions was not always significant, other factors presumably also played a role, the most likely one being shortening-induced force depression. The fact that the work performed and the velocity of shortening were generally not significantly different between conditions of compliance, despite significant reductions in force, makes it difficult to determine how impactful each factor was. One potential situation is that, while both shortening velocity and shortening-induced force depression perhaps only had a relatively small effect on their own, the concurrent effect of both of them simultaneously resulted in substantial losses in force.

As mentioned in Section 1.4.4, during isometric tetanic contractions, there is no fibre shortening when force is measured and thus, shortening velocity would not be a factor. However, with a twitch, the rise in force is so brief before the activation ceases that perhaps shortening velocity may impact the resultant force output at a given length. As seen in Figure 4.10, shortening velocity for contractions initiated at progressively longer lengths steadily declines as the fibre length is stretched, likely in relation to the reciprocal changes in in-series compliance. If shortening velocity might impact the peak force attained during brief activations, then all else being equal, force will be most depressed at these shorter lengths where velocity is highest, and least depressed at the longest lengths where velocity is lowest. Conceivably, this lengthdependent decrease in shortening velocity might impact the apparent optimum length of brief contractions such as twitches by shifting them further to the right than might be expected based on changes in $\mathrm{Ca}^{2+}$ sensitivity alone, as they are less negatively affected by the velocity of shortening. As described above, Holt and Williams reported significantly larger relative shifts in optimum length (twitch optimum length divided by tetanic optimum length) in a high- 
compliance condition compared to a low-compliance that apparently did not allow any muscle shortening. While we didn't see a difference in twitch optimum length or relative shift between low- and high-compliance conditions, the actual differences in velocity at the longer lengths was likely not great enough to allow observation of an effect even if this was the case. As a comparison, in some of our preliminary experiments using tetanic $100 \mathrm{~Hz}$ contractions, we were finding that the twitch optimum length was closer to $\sim 1.25-1.3$ times that of the $100 \mathrm{~Hz}$ optimum length (see Table 3.3). Conversely, in our later experiments, the twitch optimum length was only 1.16 times that of the triplet optimum length. Perhaps this difference in relative shift can be partially explained by the increased difference in shortening velocity between the two conditions. In the first case, due to the duration of the activation period, the velocity of shortening for the tetanic $100 \mathrm{~Hz}$ contractions would not play a factor in the resultant force output, whereas it may for the twitches. Therefore, the length-dependent decrease in shortening velocity may impact the length at which force was maximized for the twitch contractions, but not those of the $100 \mathrm{~Hz}$ contractions, pushing the optimum length of the twitches slightly farther to the right relative to the $100 \mathrm{~Hz}$ optimum length. In the other case, triplets and twitches would both be affected by the length-dependent decrease in shortening velocity, such that optimum length of both conditions might be slightly pushed to the right, causing the relative difference in optimum length between them to be smaller.

Holt and Williams (2018) reported a shift in optimum length between twitch and tetanic contractions of $\sim 1.4$ in a high compliance condition, but only $\sim 1.1$ in an apparently isometric condition. Even with a high series compliance, the velocity of shortening would not be expected to play a factor in the force output of a tetanic contraction, but with a twitch, it may. Thus, the tetanic contraction may not be affected by the decrease in shortening velocity that occurs as the 
muscle is stretched to longer lengths, but the twitch likely would, again shifting the optimum length to longer lengths relative to the tetanic contraction. When both tetanic and twitch contractions are not allowed to shorten (i.e. they are truly isometric, as Holt and Williams claimed for their low compliance condition), then obviously shortening velocity would not be a factor in force output. In this case, there would be no length dependent decrease in shortening velocity, and so any changes in optimum length between the twitch and tetanic conditions would likely be purely due to length dependent increases in $\mathrm{Ca}^{2+}$ sensitivity. Seen this way, it seems a reasonable hypothesis that the results reported by Holt and Williams were perhaps not due to the effects of shortening-induced force depression, but perhaps due to the effects of a lengthdependent decrease in shortening velocity. Because in our setup neither condition was truly isometric, and the relationship between length and shortening velocity was similar between conditions of compliance, perhaps the difference in shortening velocity between conditions of compliance was not great enough for this to change the length at which peak force occurred between conditions. While this is only conjecture, it would help to make sense of some of the results of Holt and Williams (2018), and should be explored further.

Another reason why we may not have seen any shift in optimum length despite seeing evidence of shortening-induced force depression relates to the multi-factorial nature of our experimental setup. As described in Chapter 3, there have been reports of a length dependent increase in force depression, as for a given magnitude of shortening, force depression was seen to be greater at longer lengths (see Figure 2.5). This would seem to provide the potential for a shift in optimum length, however we did not observe this. Crucially, as we were not controlling the absolute magnitude of shortening but only the relative increase via greater compliance, the 
shortening magnitude was free to vary due to other factors at play. We saw earlier that both force and compliance decreased at longer lengths; the combination of these two factors would function to reduce the magnitude of shortening as the muscle was stretched to longer lengths, which was shown in Figure 4.8. The basically opposite relationship between the previously reported lengthdependent increase in force depression and our length dependent decrease in shortening magnitude may serve to make this a zero-sum game, resulting in decreases in force with the additional in-series compliance, but no apparent change in optimum length.

\subsection{Conclusion}

Muscle is an incredibly interesting tissue to study because there are so many different factors that can influence its performance. While well-controlled studies are absolutely vital to improve our knowledge about how things work on a deeper level, in the real-world, things don't exist in isolation. In this paper we were searching for additional factors that may help explain why muscle force is maximized at a given length. As we saw, however, when one thing is changed, many other things also follow suit. For example, in our experiments, the added in-series compliance not only reduced the resultant force output, but also increased the shortening velocity. Interestingly, as touched on earlier, shortening-induced force depression is minimized at higher velocities, due to the reduction in muscle force during the shortening period. So, in our case, the increased velocity, which on its own may decrease force outputs, would also be expected to minimize any force losses that occurred by shortening-induced force depression. Perhaps the optimum length of muscle isn't only based on one particular factor, such as filament overlap, but on the complex interaction of many factors all having their own specific effect and interaction with each other. In regards to what we were exploring, a stiffer or shorter tendon would allow less shortening of the muscle fibres, and could potentially maximize force output by 
maintaining the muscle fibres in a more advantageous spot on the force-length curve, a slower shortening velocity, and less shortening induced force depression compared to a more compliant tendon. With a constant in-series compliance, these three negative effects would presumably be magnified by higher levels of activation; higher muscular force outputs would lead to larger sarcomere shortening, higher shortening velocity, and more shortening-induced force depression. In this way, maximally stimulated muscle may be relatively more "inhibited" with a more compliant tendon than submaximally stimulated muscle, where force outputs, shortening magnitudes, and shortening velocities are reduced (indeed, we saw a greater relative force reduction with triplets than with twitches). This interplay of factors may affect the force-length relationship such that the resultant optimum length may be due, not only to the degree of filament overlap, but also where on the force-length curve the combined negative effects of a higher shortening magnitude, velocity, and force depression are minimized. This would likely depend on the compliance of the SEC and how this compliance varies with changes in length.

Overall, we saw that added in-series compliance caused a significant reduction in force, which is in agreement with what others have seen (Mayfield, Launikonis, et al., 2016), but this reduction in force was not accompanied by any changes in optimum length or relative shifts in optimum length between high- and low-activation conditions. The results of Holt and Azizi (2014) and Holt and Williams (2018) remain intriguing, but we are unable to explain what they reported. We saw that neither added in-series compliance, nor the amount of work done (which relates to the degree of force depression) affected optimum length, which seems to disagree with their assertion. Thus, it is clear that further studies are required to explore this issue in more detail. 


\section{Conclusions, Limitations, and Future Directions}

\subsection{Conclusions}

Overall, we found that increased in-series compliance lead to a reduction in force regardless of activation level, but without any change in the length at which peak force occurred. The fact that force production was greatly affected by this additional compliance is an important finding with potential for real world implications. In the sporting world, the interplay of muscle and tendon mechanics has large implications for performance. For example, it has been shown that a lower Achilles tendon compliance is associated with a lower energy cost of running, and that this reduction in energy cost is due to the stiffer tendon permitting less muscle shortening during a contraction (Fletcher \& MacIntosh, 2015). Additionally, Kubo, Kawakami, \& Fukunaga (1999) explored the relationship between vastus lateralis tendon compliance and squat and countermovement jump performance, and suggested that stiffer tendons may positively impact stretch-shortening cycle exercises. It may not be as simple as "stiffer is better," however; in a simulation study, Uchida, Hicks, Dembia, \& Delp (2016) compared the estimated metabolic cost of the lower leg muscles at different speeds of running, and stated that, "when running at $2 \mathrm{~m} / \mathrm{s}$, the soleus muscle consumed less metabolic power at high tendon compliance because the strain of the tendon allowed the muscle fibers to operate nearly isometrically during stance. In contrast, the medial and lateral gastrocnemii consumed less metabolic power at low tendon compliance because less compliant tendons allowed the muscle fibers to operate closer to their optimal lengths during stance." Perhaps the most appropriate statement to make would be that, firstly, the “optimal" tendon compliance likely depends on the particular muscle-tendon-unit being 
discussed and its role in human movement, and secondly, it likely depends on the goal of the task (e.g. maximal efficiency versus maximal power or speed of movement).

\subsection{Limitations}

In terms of directly comparing these results to those of Holt and Williams' results, perhaps the chief concern is the differences in species used. There are unique differences, both physiologically as well as mechanically, between a rat medial gastrocnemius muscle tendon complex and a bullfrog plantaris muscle tendon complex. Mechanically speaking, passive force becomes substantial at much shorter lengths in mammals compared to frogs (Azizi \& Roberts, 2010). This means that, conceivably the compliance of the system in the bullfrog plantaris model was greater than in our system, even with the additional in-series compliance we added. We were somewhat limited in the range of additional compliance we could add due to shortening onto the ascending limb. If the bullfrog plantaris allowed substantially greater shortening than our setup, presumably more force depression would have occurred, which may have been more likely to result in the leftward shift in optimum length seen by Meijer et al (1997). Additionally, because of this delay in the rise of passive force, if the compliance of the bullfrog plantaris was consistently higher throughout the length excursion, this may allow a more steady degree of work performed and/or magnitude of shortening through the range of muscle lengths tested. In comparison to our scenario where the shortening magnitude decreased throughout, and the work performed dropped off steeply after the optimum length, perhaps a more consistent degree of compliance would allow the length-dependent increase in force depression to actually manifest itself in changes in optimum length, where in our system it may have been "washed out."

From our results, it was clear that the additional in-series compliance negatively impacted muscular force production. However, we were not able to confidently state the cause of the force 
depression seen. Both shortening-induced force depression, as well as an increase in velocity of shortening were suggested as potential factors, but it was difficult to ascertain the relative impact of each with our data. We calculated both shortening velocity as well as work performed from sonomicrometry data. Compared to measuring length changes and velocity from a servomotor system, the sonomicrometry data may be prone to more inherent error, which may have increased the variance of the data and weakened the relationships observed. Additionally, as mentioned in the discussion, the comparison of work performed and force produced was done at a given relative starting length, which would not account for alterations in final fascicle length brought about by the increased magnitude of shortening. While the final fascicle lengths would have been similar, this potential confounding factor would have likely weakened the relationship observed.

As detailed in Chapter 3, we dealt with a number of experimental issues early on that precluded the analysis of some of the data we obtained. Chiefly, losses in force due to fatigue or (perhaps more likely) axonal damage to the motor nerve were an issue we faced that would confound the interpretation of our results. During the final 8 experiments, fatigue was monitored via periodic twitches, and losses in force through the experiment were not apparent.

Additionally, for 6 of the 8 final experiments, the high-compliance protocol was executed first, and yet we still saw substantial reductions in force. Thus, we are confident that the force losses seen were a real effect, and not an artifact of force losses due to fatigue or damage to the nerve.

Regarding the estimated optimum length, unfortunately our analysis would likely not be sensitive enough to detect minute changes in optimum length between the low- and highcompliance conditions. For comparison's sake, Meijer et al (1997) detected significant changes in optimum length amounting to $\sim 1 \%$ of the isometric optimum length. In our setup, the 
sonometric crystal data had some inherent inconsistency that may have increased the variability in the force-length curves. The polynomial curve fitting of these data points may have been impacted whereby the estimated optimum length could have been slightly "pulled" over by even a single anomalous data point. In this way, if there was a length-dependent increase in force depression that caused only a very small shift in optimum length, it would have been difficult to detect in our setup. Nevertheless, even if we had been able to improve the sensitivity of our setup, this doesn't change the fact that our results were substantially different than those of Holt and Williams (2018), showing that more research is clearly needed to understand the discrepancies seen.

\subsection{Future Directions}

In my research here, I offered a question as to how impactful the increased shortening velocity allowed by the additional compliance was on the reductions in force output for nontetanic contractions, and whether this may impact the shift in optimum length of twitch contractions compared to tetanic contractions. Due to the multiple factors involved in our setup, I unfortunately was not able to provide a conclusive answer to this question. With a more wellcontrolled study, I think determining the answer to this question may provide some insight to the results of Holt and Williams (2018). Namely, in their low-compliance (isometric) condition, the twitch optimum length was only $\sim 8 \%$ longer than the tetanic optimum length. In their highcompliance condition the twitch optimum length was $\sim 38 \%$ greater than the tetanic optimum length. If the compliant bullfrog plantaris tendon that they used for their "high-compliance" condition allowed a significant increase in shortening velocity over the isometric condition, and this shortening velocity decreased over the range of lengths (as I saw in my data), then perhaps the increased relative shift in optimum length that they saw in the high-compliance condition 
related to the length-dependent decrease in velocity, thereby allowing relatively greater force output at longer lengths than what one would expect based on $\mathrm{Ca}^{2+}$-dependent effects alone. In my setup, perhaps the difference in shortening velocity between the low- and high-compliance conditions was not great enough for this to be a factor.

I was unfortunately unable to explain the intriguing results of either Holt and Williams (2018) or Holt and Azizi (2014). Most curiously, the fact that they saw a relative shift in optimum length while only decreasing stimulation voltage remains somewhat of a mystery. It has been suggested that perhaps submaximal voltage still effectively decreases $\left[\mathrm{Ca}^{2+}\right]$ in some of the active motor units if the electrical current only intermittently reaches some of the motor nerve axons (essentially reducing their firing frequency) (Herzog, personal communication, 2018). This notion has not been tested, however, and so may provide some answers. Yet, as I found out, with a consistent connection quality between the electrode cuff and the nerve, I did not see any shift in optimum length. Thus, if this mechanism were able to explain some of the results, it would likely be dependent on the quality of the connection between the nerve and the electrodes and so may be difficult to consistently test. In any case, the disparity between the results reported here and those of Holt and Azizi (2014) and Holt and Williams (2018) illustrate the need for additional work to be done. 


\section{References}

Azizi, E., \& Roberts, T. J. (2010). Muscle performance during frog jumping: Influence of elasticity on muscle operating lengths. Proceedings of the Royal Society B: Biological Sciences, 277(1687), 1523-1530. https://doi.org/10.1098/rspb.2009.2051

Balnave, C. D., \& Allen, D. G. (1996). The effect of muscle length on intracellular calcium and force in single fibres from mouse skeletal muscle. Journal of Physiology. https://doi.org/10.1113/jphysiol.1996.sp021339

Blix, M. (1891). Die Lange und die Spannung des Muskels. Skandinavisches Archiv Fur Physiologie, 3, 295-318.

Boulpaep, E., Boron, W., Caplan, M., Cantley, L., Igarashi, P., Aronson, P., \& Moczydlowski, E. (2009). Medical Physiology a Cellular and Molecular Approach. Signal Transduct.

Burkholder, T. J., \& Lieber, R. L. (2001). Sarcomere length operating range of vertebrate muscles during movement. Journal of Experimental Biology.

Caputo, C., Gerday, C., Lopez, J. R., Taylor, S. R., \& Bolaños, P. (1998). Opposite effects of cooling on twitch contractions of skeletal muscle isolated from tropical toads (Leptodactylidae) and northern frogs (Ranidae). Journal of Comparative Physiology - B Biochemical, Systemic, and Environmental Physiology. https://doi.org/10.1007/s003600050182

Cornachione, A. S., Benedini-Elias, P. C. O., Polizello, J. C., Carvalho, L. C., \& MattielloSverzut, A. C. (2011). Characterization of fiber types in different muscles of the hindlimb in female weanling and adult wistar rats. Acta Histochemica et Cytochemica. https://doi.org/10.1267/ahc.10031

Doi, Y. (1920). Studies on muscular contraction: I. The influence of temperature on the 
mechanical performance of skeletal and heart muscle. The Journal of Physiology, 54(4), 218-226. https://doi.org/10.1113/jphysiol.1920.sp001921

Doi, Y. (1921). Studies on muscular contraction: II. The relation between the maximal work and the tension developed in a muscle twitch, and the effects of temperature and extension. The Journal of Physiology, 54(5-6), 335-341. https://doi.org/10.1113/jphysiol.1921.sp001934

Endo, M. (1972). Stretch induced Increase in Activation of Skinned Muscle Fibres by Calcium. Nature, 237(76), 211-213. https://doi.org/10.1038/239137a0

Enoka, R. M., \& Duchateau, J. (2017). Rate coding and the control of muscle force. Cold Spring Harbor Perspectives in Medicine. https://doi.org/10.1101/cshperspect.a029702

Evans, C. L., \& Hill, A. V. (1914). The relation of length to tension development and heat production on contraction in muscle. The Journal of Physiology, 49(1-2), 10-16. https://doi.org/10.1113/jphysiol.1914.sp001684

Fletcher, J. R., \& MacIntosh, B. R. (2015). Achilles tendon strain energy in distance running: Consider the muscle energy cost. Journal of Applied Physiology, 118(2), 193-199. https://doi.org/10.1152/japplphysiol.00732.2014

Gandevia, S. C. (2001). Spinal and supraspinal factors in human muscle fatigue. Physiological Reviews. https://doi.org/10.1152/physrev.2001.81.4.1725

Gordon, A. M., Huxley, A. F., \& Julian, F. J. (1966). The variation in isometric tension with sarcomere length in vertebrate muscle fibres. Journal of Physiology, 184, 170-192.

Grange, R. W., Vandenboom, R., \& Houston, M. E. (1993). Physiological significance of myosin phosphorylation in skeletal muscle. Canadian Journal of Applied Physiology $=$ Revue Canadienne de Physiologie Appliquée. https://doi.org/10.1139/h93-020

Granzier, H. L., \& Pollack, G. H. (1989). Effect of active pre-shortening on isometric and 
isotonic performance of single frog muscle fibres. The Journal of Physiology. https://doi.org/10.1113/jphysiol.1989.sp017723

Hepple, R. T. (2002). The role of O2 supply in muscle fatigue. Canadian Journal of Applied Physiology. https://doi.org/10.1139/h02-004

Herzog, W., Leonard, T. R., \& Wu, J. Z. (2000). The relationship between force depression following shortening and mechanical work in skeletal muscle. Journal of Biomechanics. https://doi.org/10.1016/S0021-9290(00)00008-7

Herzog, Walter. (2018). The multiple roles of titin in muscle contraction and force production. Biophysical Reviews. https://doi.org/10.1007/s12551-017-0395-y

Herzog, Walter, Guimaraes, A. C., Anton, M. G., \& Carter-Erdman, K. A. (1991). Momentlength relations of rectus femoris muscles of speed skaters/cyclists and runners. Medicine and Science in Sports and Exercise. https://doi.org/10.1249/00005768-199111000-00015

Holt, N. C., \& Azizi, E. (2014). What drives activation-dependent shifts in the force - Length curve? Biology Letters, 10(9). https://doi.org/10.1098/rsbl.2014.0651

Holt, N., \& Williams, C. (2018). Can strain dependent inhibition of cross- bridge binding explain shifts in optimum muscle length? Integrative and Comparitive Biology, (June). https://doi.org/10.1093/icb/icy050/5032872

Huxley, A. (1957). Muscle structure and theories of contraction. Prog Biophys Biophys Chem., 7, $257-318$.

Huxley, A. F., \& Niedergerke, R. (1954). Structural changes in muscle during contraction: Interference microscopy of living muscle fibres. Nature. https://doi.org/10.1038/173971a0 Huxley, H., \& Hanson, J. (1954). Changes in the Cross-striations of muscle during contraction and stretch and their structural interpretation. Nature. https://doi.org/10.1038/173973a0 
Julian, F. J., Moss, R. L., \& Sollins, M. R. (1978). The mechanism for vertebrate striated muscle contraction. Circulation Research, 42(1), 2-14. https://doi.org/10.1161/01.RES.42.1.2

Kerschensteiner, M., Schwab, M. E., Lichtman, J. W., \& Misgeld, T. (2005). In vivo imaging of axonal degeneration and regeneration in the injured spinal cord. Nature Medicine. https://doi.org/10.1038/nm1229

Kössler, F., Lange, F., \& Küchler, G. (1987). Isometric twitch and tetanic contraction of frog skeletal muscles at temperatures between 0 to 30 degrees C. Biomedica Biochimica Acta.

Kubo, K., Kawakami, Y., \& Fukunaga, T. (1999). Influence of elastic properties of tendon structures on jump performance in humans. Journal of Applied Physiology, 87(6), 20902096. https://doi.org/10.1152/jappl.1999.87.6.2090

MacIntosh, B. R., \& MacNaughton, M. B. (2005). The length dependence of muscle active force: considerations for parallel elastic properties. Journal of Applied Physiology, 98(5), 16661673. https://doi.org/10.1152/japplphysiol.01045.2004

MacIntosh, Brian R. (2017). Recent developments in understanding the length dependence of contractile response of skeletal muscle. European Journal of Applied Physiology, 117(6), 1059-1071. https://doi.org/10.1007/s00421-017-3591-3

MacIntosh, Brian R., Esau, S. P., Holash, R. J., \& Fletcher, J. R. (2011). Procedures for rat in situ skeletal muscle contractile properties. Journal of Visualized Experiments. https://doi.org/10.3791/3167

MacIntosh, Brian R., \& Rassier, D. E. (2002). What is fatigue? Canadian Journal of Applied Physiology. https://doi.org/10.1139/h02-003

MacNaughton, M. B., Campbell, J. J., \& MacIntosh, B. R. (2007). Dantrolene, like fatigue, has a length-dependent effect on submaximal force-length relationships of rat gastrocnemius 
muscle. Acta Physiologica, 189(3), 271-278. https://doi.org/10.1111/j.17481716.2006.01645.x

Mayfield, D. L., Cresswell, A. G., \& Lichtwark, G. A. (2016). Effects of series elastic compliance on muscle force summation and the rate of force rise. The Journal of Experimental Biology, 219(20), 3261-3270. https://doi.org/10.1242/jeb.142604

Mayfield, D. L., Launikonis, B. S., Cresswell, A. G., \& Lichtwark, G. A. (2016). Additional inseries compliance reduces muscle force summation and alters the time course of force relaxation during fixed-end contractions. The Journal of Experimental Biology, 219(22), 3587-3596. https://doi.org/10.1242/jeb.143123

Meijer, K., Grootenboer, H. J., Koopman, H. F. J. M., \& Huijing, P. A. (1996). Linear description of shortening induced changes in isometric length-force characteristics of rat muscle. Annual International Conference of the IEEE Engineering in Medicine and Biology - Proceedings, 2, 567-568. https://doi.org/10.1109/iembs.1996.651867

Meijer, Kenneth, Grootenboer, H. J., Koopman, B. F. J. M., \& Huijing, P. A. (1997). Fully isometric length-force curves of rat muscle differ from those during and after concentric contractions. Journal of Applied Biomechanics, 13(2), 164-181. https://doi.org/10.1123/jab.13.2.164

Murthy, G., Hargens, A. R., Lehman, S., \& Rempel, D. M. (2001). Ischemia causes muscle fatigue. Journal of Orthopaedic Research. https://doi.org/10.1016/S0736-0266(00)90019-6

Muscle, L. O. F. (1938). The heat of shortening and the dynamic constants of muscle. Proceedings of the Royal Society of London. Series B - Biological Sciences, 126(843), 136195. https://doi.org/10.1098/rspb.1938.0050

Piazzesi, G., Reconditi, M., Linari, M., Lucii, L., Bianco, P., Brunello, E., ... Lombardi, V. 
(2007). Skeletal Muscle Performance Determined by Modulation of Number of Myosin Motors Rather Than Motor Force or Stroke Size. Cell, 131(4), 784-795. https://doi.org/10.1016/j.cell.2007.09.045

Podlubnaya, Z., Kạkol, I., Moczarska, A., Stępkowski, D., \& Udaltsov, S. (1999). Calciuminduced structural changes in synthetic myosin filaments of vertebrate striated muscles. Journal of Structural Biology. https://doi.org/10.1006/jsbi.1999.4129

Rack, P. M., \& Westbury, D. R. (1969). The effects of length and stimulus rate on tension in the isometric cat soleus muscle. Journal of Physiology, 204, 443-460.

Rall, J. A. (1996). Role of parvalbumin in skeletal muscle relaxation. News in Physiological Sciences. https://doi.org/10.1152/physiologyonline.1996.11.6.249

Ramsey, R. W. \& Street, S. F. (1940). The isometric length-tension diagram of isolated skeletal muscle fibers of the frog. Journal of Cellular and Comparative Physiology, 15(1), 11-34. https://doi.org/10.1007/BF01600864

Rassier, D. E., MacIntosh, B. R., \& Herzog, W. (1999). Length dependence of active force production in skeletal muscle. Journal of Applied Physiology, 86(5), 1445-1457.

Rassier, Dilson E, \& Macintosh, B. R. (2002). Sarcomere length-dependence of activitydependent twitch potentiation in mouse skeletal muscle. BMC Physiology, 8, 1-8.

Sieck, G. C., \& Prakash, Y. S. (1995). Fatigue at the neuromuscular junction: Branch point vs. presynaptic vs. postsynaptic mechanisms. Advances in Experimental Medicine and Biology.

Stephenson, D. G., \& Williams, D. A. (1982). Effects of sarcomere length on the force-pCa relation in fast- and slow-twitch skinned muscle fibres from the rat. The Journal of Physiology, 333(1), 637-653. https://doi.org/10.1113/jphysiol.1982.sp014473

Stephenson, D. G., \& Williams, D. A. (1985). Temperature-dependent calcium sensitivity 
changes in skinned muscle fibres of rat and toad. J. Physiol., 360, 1-12.

Stienen, G. J. M., Blangé, T., \& Treijtel, B. W. (1985). Tension development and calcium sensitivity in skinned muscle fibres of the frog. Physiology, 405, 19-23.

Sun, Y. B., Lou, F., \& Edman, K. A. P. (1996). The relationship between the intracellular Ca2+ transient and the isometric twitch force in frog muscle fibres. Experimental Physiology, 81(5), 711-724. https://doi.org/10.1113/expphysiol.1996.sp003971

Sundberg, C. W., \& Fitts, R. H. (2019). Bioenergetic basis of skeletal muscle fatigue. Current Opinion in Physiology. https://doi.org/10.1016/j.cophys.2019.05.004

Taylor, S. R., \& Rudel, R. (1970). Striated muscle fibers: Inactivation of contraction induced by shortening. Science. https://doi.org/10.1126/science.167.3919.882

Uchida, T. K., Hicks, J. L., Dembia, C. L., \& Delp, S. L. (2016). Stretching your energetic budget: How tendon compliance affects the metabolic cost of running. PLoS ONE, 11(3), 119. https://doi.org/10.1371/journal.pone.0150378

Vaz, M. A., De La Rocha Freitas, C., Leonard, T., \& Herzog, W. (2012). The force-length relationship of the cat soleus muscle. Muscles, Ligaments and Tendons Journal, 2(2), 7984.

Wang, J. T., Medress, Z. A., \& Barres, B. A. (2012). Axon degeneration: Molecular mechanisms of a self-destruction pathway. Journal of Cell Biology. https://doi.org/10.1083/jcb.201108111

Williams, C. D., Salcedo, M. K., Irving, T. C., Regnier, M., \& Daniel, T. L. (2013). The lengthtension curve in muscle depends on lattice spacing. Proceedings of the Royal Society B: Biological Sciences, 280(1766), 1-7. https://doi.org/10.1098/rspb.2013.0697

Xu, S., Offer, G., Gu, J., White, H. D., \& Yu, L. C. (2003). Temperature and ligand dependence 
of conformation and helical order in myosin filaments. Biochemistry, 42(2), 390-401. https://doi.org/10.1021/bi026085t 


\title{
Appendix A - Copyright Permissions
}

\section{Permission for Figure 1.1 and Figure 1.2}

\author{
SECTION 1 \\ Terms of Use for Everyone
}

Ownership. The content on the Services is the property of Britannica, its affiliated companies or licensors, and is protected by international copyright, patent, and trademark laws.

\begin{abstract}
Advertising. Advertisements, promotions, and marketing messages may appear on the Services from time to time, unless you or your institution has a subscription-based Service. Please see our Privacy Policy for more information.

Use of Content. You may display, reproduce, print or download content on the Services only for your personal, non-commercial use. If you are a teacher, scholar or student, you may copy reasonable portions of the content for lesson plans, interactive whiteboards, reports, dissertations, presentations, school newspapers and for similar nonprofit educational purposes to the extent permitted by applicable law. In each case, however, you may not remove or alter any copyright, trademark, service mark or other proprietary notices or legends. You may not publish, distribute, retransmit, sell or provide access to the content on the Services, except as permitted under applicable law or as described in these Terms of Use. Britannica works to ensure that all the content on its Services is in compliance with applicable U.S. copyright laws. However, in the case of works on the Services authored by parties other than Britannica, you may wish to check on their copyright status before downloading them if you are in another country. You may not use data mining, robots, screen scraping, or similar data gathering and extraction tools on the Services, except with our express written permission. You may not decompile, reverse engineer or disassemble any software or other products or processes accessible through the Services, insert any code or product, or manipulate the content of the Services in any way that affects the user's experience.
\end{abstract}

\section{RE: Britannica Syndication Inquiry Form}

WS Wallace, Sylvia <SWallace@eb.com> 9:42 AM

To: Keenan MacDougall

Dear Keenan, Encyclopædia Britannica is happy to grant you permission to use and adapt our illustrations "striated muscle structure" and "actin and myosin filaments" only in your non-commercial dissertation. We will waive the usual fee and just ask that you include the following credits in your paper:

For striated muscles: "By courtesy of Encyclopædia Britannica, Inc., copyright 2015; used with permission" For filaments: “By courtesy of Encyclopædia Britannica, Inc., copyright 2012; used with permission”

Best regards, Sylvia 


\title{
Permission for Figure 1.4
}

\author{
JOHN WILEY AND SONS LICENSE \\ TERMS AND CONDITIONS
}

Jan 24,2020

This Agreement between Keenan MacDougall ("You") and John Wiley and Sons ("John Wiley and Sons") consists of your license details and the terms and conditions provided by John Wiley and Sons and Copyright Clearance Center.

License Number $\quad 4747411000156$

License date Jan 13, 2020

Licensed Content Publisher

Licensed Content Publication

Licensed Content Title

Licensed Content Author
John Wiley and Sons

Journal of Physiology

The variation in isometric tension with sarcomere length in vertebrate muscle fibres

F. J. Julian, A. F. Huxley, A. M. Gordon 


\section{Permission for Figure 1.6}

\section{JOHN WILEY AND SONS LICENSE \\ TERMS AND CONDITIONS}

Jan 24,2020

This Agreement between Keenan MacDougall ("You") and John Wiley and Sons ("John Wiley and Sons") consists of your license details and the terms and conditions provided by John Wiley and Sons and Copyright Clearance Center.

License Number $\quad 4754400838894$

License date Jan 22, 2020

Licensed Content

Publisher

John Wiley and Sons

Licensed Content

Publication

Experimental Physiology

Licensed Content The relationship between the intracellular $\mathrm{Ca} 2$ transient and the Title isometric twitch force in frog muscle fibres

Licensed Content Author

KA Edman, F Lou, YB Sun 


\section{Permission for Figure 2.1}

\section{JOHN WILEY AND SONS LICENSE TERMS AND CONDITIONS}

Jan 24,2020

This Agreement between Keenan MacDougall ("You") and John Wiley and Sons ("John Wiley and Sons") consists of your license details and the terms and conditions provided by John Wiley and Sons and Copyright Clearance Center.

License Number $\quad 4754630891573$

License date Jan 23, 2020

Licensed Content Publisher

John Wiley and Sons

Licensed Content Publication

Journal of Physiology

Licensed Content Title

Studies on muscular contraction

Licensed Content Author

Yasukazu Doi 


\section{Permission for Figure 2.2}

\section{JOHN WILEY AND SONS LICENSE \\ TERMS AND CONDITIONS}

Jan 24,2020

This Agreement between Keenan MacDougall ("You") and John Wiley and Sons ("John Wiley and Sons") consists of your license details and the terms and conditions provided by John Wiley and Sons and Copyright Clearance Center.

License Number $\quad 4726121309212$

License date $\quad$ Dec 11, 2019

Licensed Content Publisher

Licensed Content Publication

Licensed Content Title

Licensed Content Author
John Wiley and Sons

Journal of Physiology

Temperature-dependent calcium sensitivity changes in skinned muscle fibres of rat and toad.

D A Williams, D G Stephenson 


\section{Permission for Figure 2.3}

\section{JOHN WILEY AND SONS LICENSE \\ TERMS AND CONDITIONS}

Jan 24,2020

This Agreement between Keenan MacDougall ("You") and John Wiley and Sons ("John Wiley and Sons") consists of your license details and the terms and conditions provided by John Wiley and Sons and Copyright Clearance Center.

License Number $\quad 4726121142501$

License date $\quad$ Dec 11, 2019

Licensed Content Publisher

John Wiley and Sons

Licensed Content Publication

Journal of Physiology

Licensed Content Effects of sarcomere length on the force- $\mathrm{pCa}$ relation in fast- and Title slow-twitch skinned muscle fibres from the rat

Licensed Content Author

D. A. Williams, D. G. Stephenson 


\section{Permission for Figure 2.4}

The Royal Society (U.K.) - License Terms and Conditions

This is a License Agreement between Keenan MacDougall ("You") and The Royal Society (U.K.) ("Publisher") provided by Copyright Clearance Center ("CCC"). The license consists of your order details, the terms and conditions provided by The Royal Society (U.K.), and the CCC terms and conditions.

All payments must be made in full to CCC.

Order Date

Order license ID

ISSN

LICENSED CONTENT

Publication Title

Author/Editor

Date

Language
1015055-1

$1471-2954$

\section{4-Jan-2020}

Type of Use

Publisher

Portion

Country

Proceedings. Biological sciences

Royal Society (Great Britain)

12/31/1996

English
Rightsholder Publication Type

URL
Republish in a thesis/dissertation

ROYAL SOCIETY

Chart/graph/table/figure

United Kingdom of Great Britain and Northern Ireland

The Royal Society (U.K.)

e-Journal

https://royalsociety.org/journals/

\section{REUSE CONTENT DETAILS}

Title, description or numeric reference of the portion(s)

Editor of portion(s)

Volume of serial or monograph

Page or page range of portion
Figure $1 \mathrm{~b}$

N/A

280
Title of the article/chapter the portion is

from

Author of portion(s)

Issue, if republishing an article from a

serial

Publication date of portion
The length-tension curve in muscle depends on lattice spacing

C. David Williams, Mary K. Salcedo, Thomas C. Irving, Michael Regnier and Thomas L.

N/A

2013-09-07 


\section{Permission for Figure 2.5}

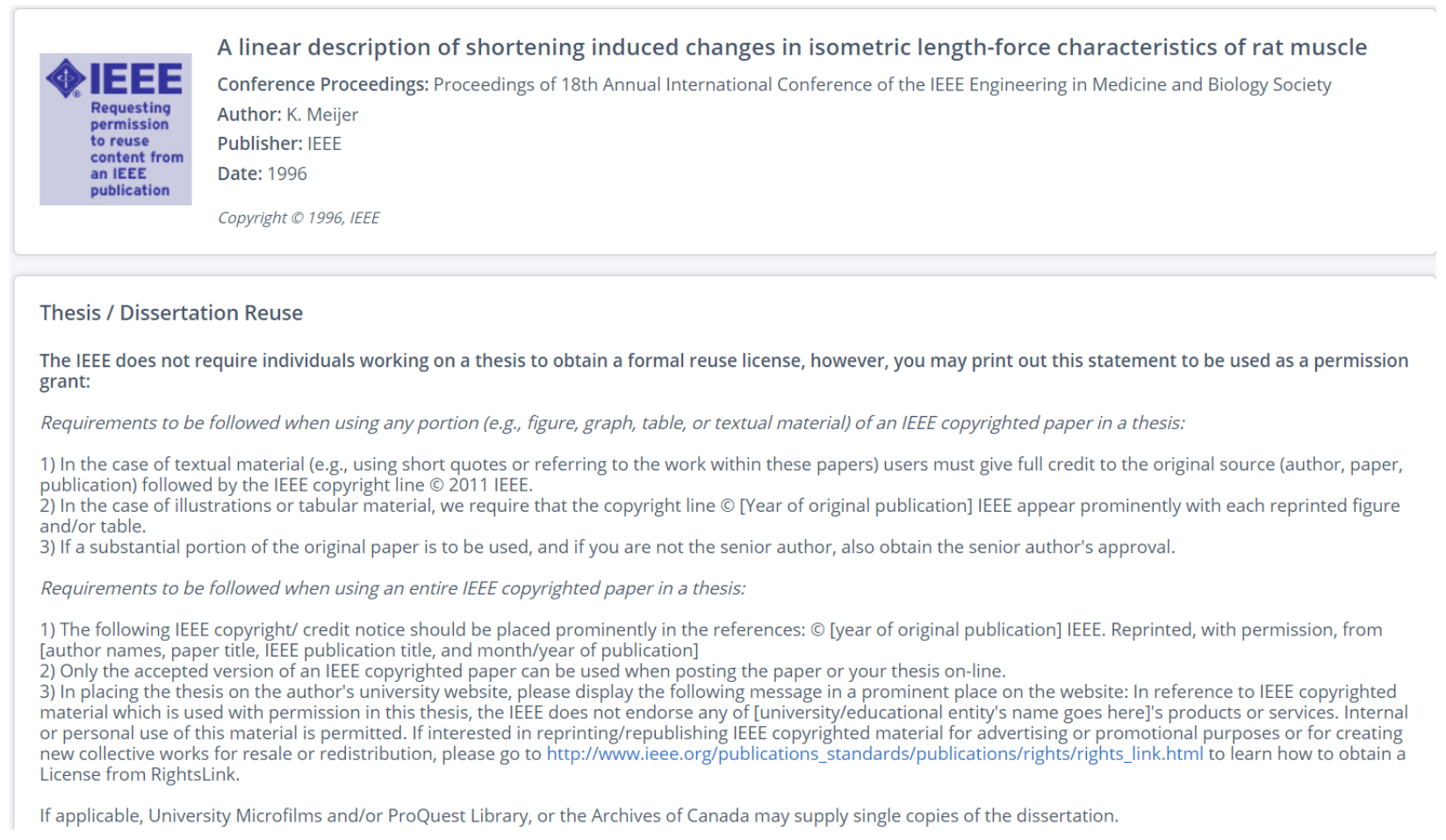




\section{Permission for Figure 2.6}

Human Kinetics, Inc. - License Terms and Conditions

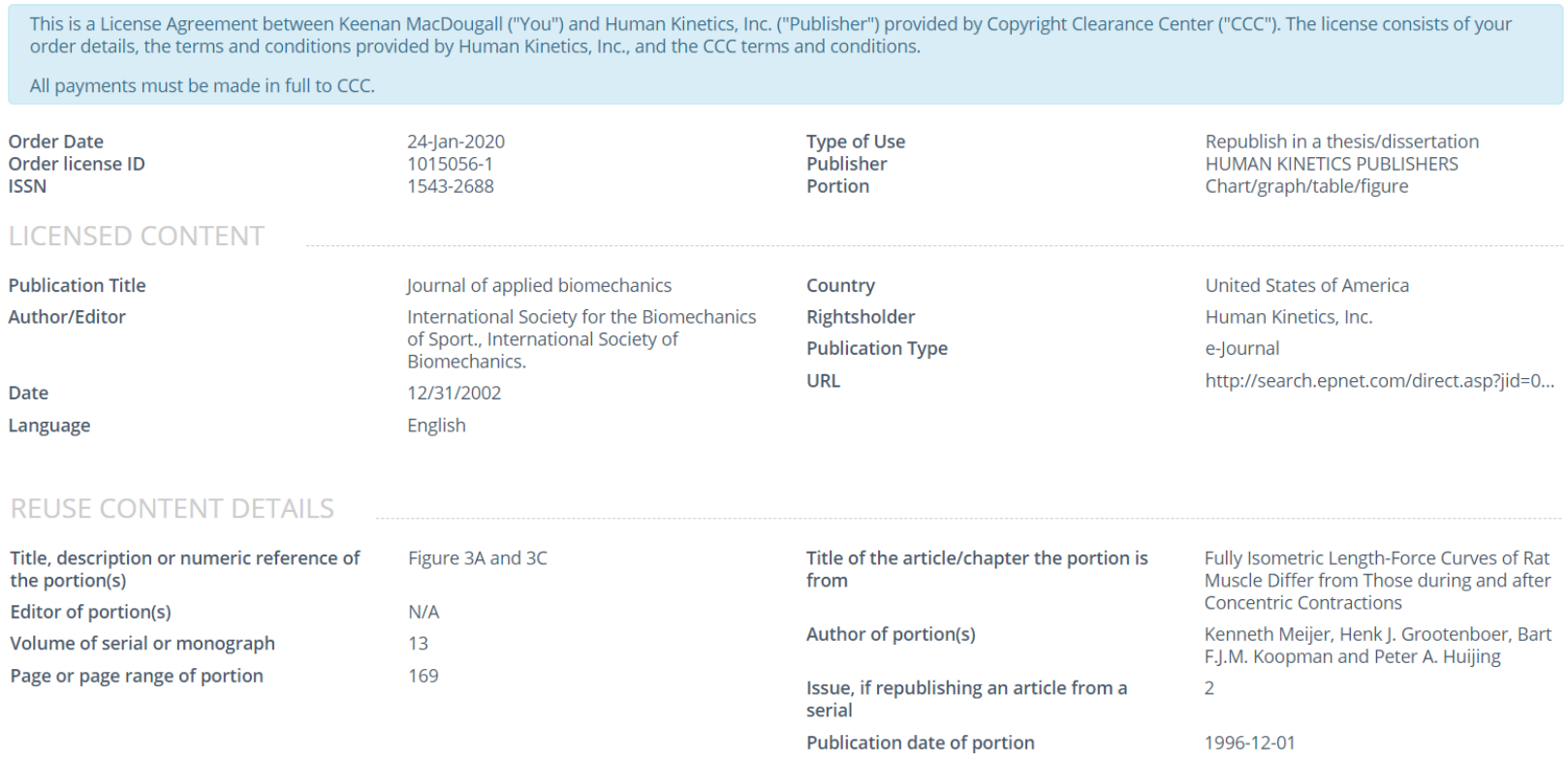




\section{Permission for Figure 2.7}

THE AMERICAN PHYSIOLOGICAL SOCIETY LICENSE TERMS AND CONDITIONS

Jan 24,2020

This Agreement between Keenan MacDougall ("You") and The American Physiological Society ("The American Physiological Society") consists of your license details and the terms and conditions provided by The American Physiological Society and Copyright Clearance Center.

License Number $\quad 4754841203947$

License date $\quad \operatorname{Jan} 23,2020$

Licensed Content

Publisher

The American Physiological Society

Licensed Content

Publication

Journal of Applied Physiology

Licensed Content Title

The length dependence of muscle active force: considerations for parallel elastic properties

Licensed Content Author

Brian R. MacIntosh, Meredith B. MacNaughton 
